NIST Technical Note 2028

\title{
Community Risk Ratings for the FireCARES System
}

Dr. Stanley Gilbert

This publication is available free of charge from: https://doi.org/10.6028/NIST.TN.2028

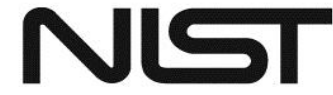


NIST Technical Note 2028

\title{
Community Risk Ratings for the FireCARES System
}

\author{
Dr. Stanley Gilbert \\ Office of Applied Economics \\ Engineering Laboratory
}

This publication is available free of charge from:

https://doi.org/10.6028/NIST.TN.2028

October 2018

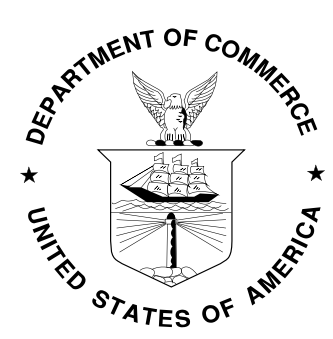

U.S. Department of Commerce

Wilbur L. Ross, Jr., Secretary

National Institute of Standards and Technology Walter Copan, NIST Director and Undersecretary of Commerce for Standards and Technology 
Certain commercial entities, equipment, or materials may be identified in this document in order to describe an experimental procedure or concept adequately. Such identification is not intended to imply recommendation or endorsement by the National Institute of Standards and Technology, nor is it intended to imply that the entities, materials, or equipment are necessarily the best available for the purpose.

National Institute of Standards and Technology Technical Note 2028

Natl. Inst. Stand. Technol. Tech. Note 2028, 76 pages (October 2018) CODEN: NTNOEF

This publication is available free of charge from: https://doi.org/10.6028/NIST.TN.2028 


\begin{abstract}
The FireCARES (Community Assessment Response Evaluation System) project is a web-based tool intended to help fire departments effectively match resources to community risk. Among other things, the analysis includes estimates of the community risk and estimates of effectiveness of response. This report documents the estimation of the community risk for fires, fire injuries, fire deaths, and emergency medical services calls. The community risk for fires is broken out by low- medium- and high-hazard properties as defined in NFPA Standard 1710.
\end{abstract}

\title{
Key words
}

Fire Risk; FireCARES; Fire; Injury; Death; EMS 
Table of Contents

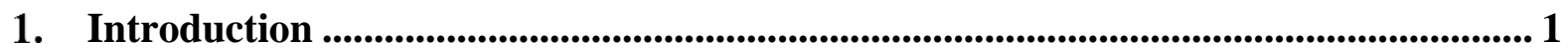

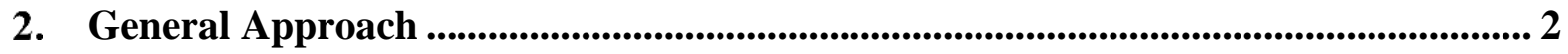

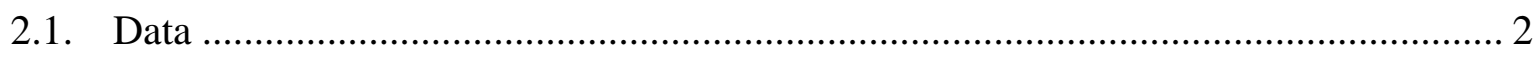

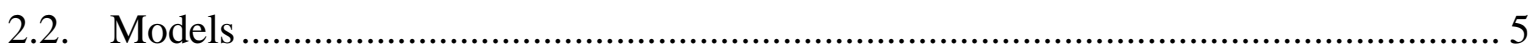

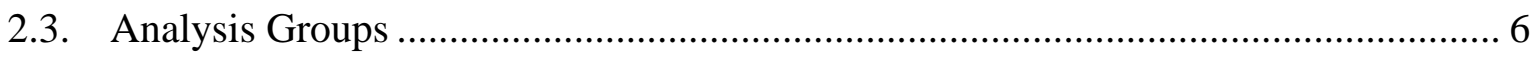

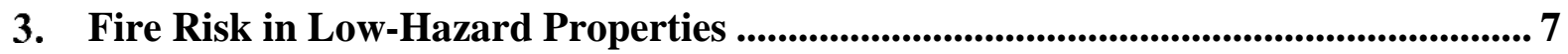

4. Fire Risk in Medium-Hazard Properties.................................................................9

5. Fire Risk in High-Hazard Properties................................................................... 11

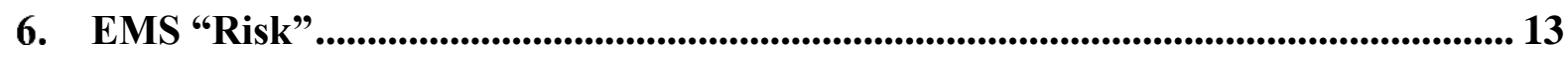

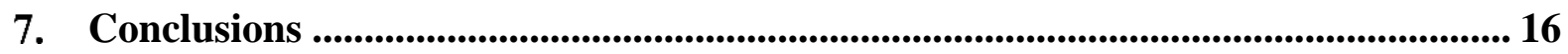

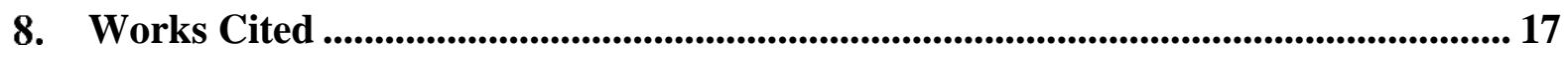

Appendix A: Variables and Data Sources ................................................................. 18

Appendix B: Low- Hazard Properties: Model Results....................................................... 22

Appendix C: Medium- Hazard Properties: Model Results................................................ 27

Appendix D: High-Hazard Properties: Model Results .................................................... 37

Appendix E: EMS Model Results .................................................................................. 52 


\section{List of Tables}

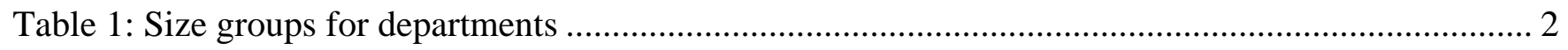

Table 2: Small Filters: Specific Definitions...................................................................................... 4

Table 3: Percent of records removed for each filter by property hazard level. .......................................... 4

Table 4:Predictor groups used for low-hazard property models............................................................ 7

Table 5:Predictor groups used for medium-hazard property models...................................................... 9

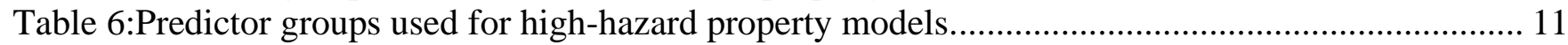

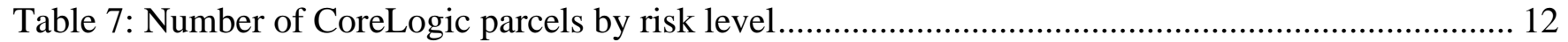

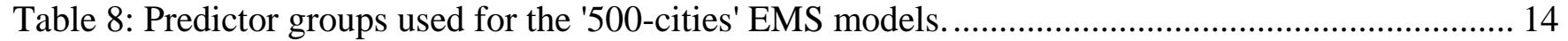

Table 9: Predictor groups used for the 'County' EMS models.............................................................. 15

Table 10: Best models and models selected for FireCARES production use........................................... 16

Table 11: Root Mean Square (RMS) Errors of models of fire risk for low hazard properties.................. 22

Table 12: RMS Errors of models of percent of fires that grow beyond the room of origin for low hazard

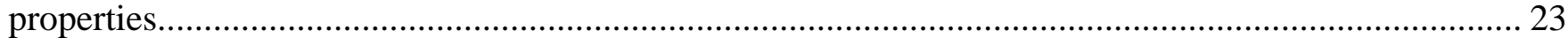

Table 13: RMS Errors of models of percent of fires that grow beyond the structure of origin for low

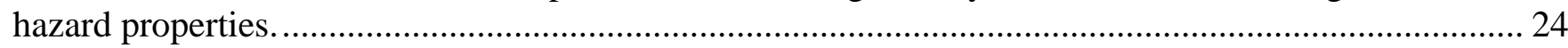

Table 14: RMS Errors of models of fire injury for low hazard properties. ............................................. 25

Table 15: RMS Errors of models of fire deaths for low hazard properties................................................ 26

Table 16: RMS Errors of models of fire risk for medium hazard properties............................................. 27

Table 17: RMS Errors of models of percent of fires that grow beyond the room of origin for medium hazard properties.

Table 18: RMS Errors of models of percent of fires that grow beyond the structure of origin for medium

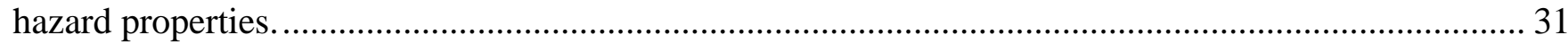

Table 19: RMS Errors of models of fire injury for medium hazard properties. ........................................ 33

Table 20: RMS Errors of models of fire deaths for medium hazard properties. ........................................ 35

Table 21: RMS Errors of models of fire risk for high hazard properties................................................ 37

Table 22: RMS Errors of models of percent of fires that grow beyond the room of origin for high hazard

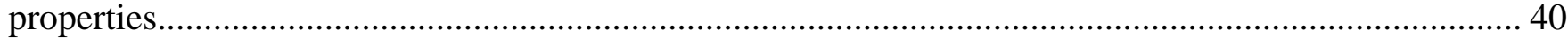

Table 23: RMS Errors of models of percent of fires that grow beyond the structure of origin for high

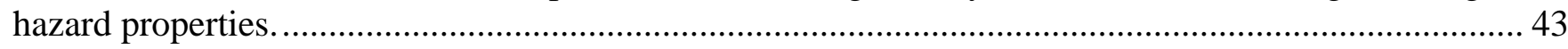

Table 24: RMS Errors of models of fire injuries for high hazard properties............................................ 46

Table 25: RMS Errors of models of fire deaths for high hazard properties. .............................................. 49

Table 26: RMS Errors of the models for EMS risk based on the 500-cities data set. .............................. 52

Table 27:RMS Errors of the models for EMS risk based on the county health data set. ......................... 58

\section{List of Figures}

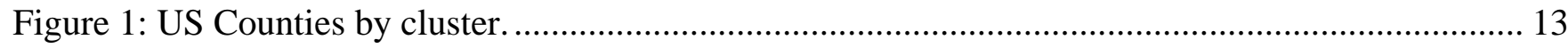


Abbreviations:

ACS: $\quad$ American Community Survey

BRFSS: Behavioral Risk Factor Surveillance System

CDC: $\quad$ (US) Centers for Disease Control

EMS Emergency Medical Services

FireCARES: Community Assessment Response Evaluation System

GLM: $\quad$ Generalized Linear Model

LASSO: Least Absolute Shrinkage and Selection Operator

NFIRS National Fire Incident Reporting System

NHIS: $\quad$ National Health Interview Survey

NFPA National Fire Protection Association

RMS Root Mean Square

RMSE Root Mean Square Error 


\section{Introduction}

The FireCARES project (www.firecares.org) is a web-based tool intended to help fire departments effectively match resources to community risk. Among other things, the analysis includes estimates of the community risk and estimates of effectiveness of response. This report describes the estimation of the community risk.

A literature review of the factors affecting death and injury in fires is in [1]. They found that the factors affecting the likelihood of death and injury in fires include age (and in particular, being older or very young), race (specifically black or American-Indian or Eskimo) gender, older homes, mobile homes, rentals, the lack of a smoke detector, and the use of alcohol and tobacco. For example, living in a lowincome household or area is associated with a higher risk of injury in fire. The use of space heaters was associated with increased risk of death in fire.

As far as I am aware, no one has attempted to estimate the spatial risk for fire, injury or death, or emergency medical services (EMS) calls at the community-level.

This report is organized as follows: Section 2 describes the data used and the general approach to estimating community risk. Section 3 describes the analysis of community fire risk for low-hazard properties. Section 4 describes the analysis of community fire risk for medium-hazard properties. Section 5 describes the analysis of community fire risk for high-hazard properties. Section 6 describes the analysis of community "risk" for EMS calls. Section 7 concludes. 


\section{General Approach}

\subsection{Data}

Data were from a number of different sources. The main data on fires and EMS calls were from the National Fire Incident Reporting System (NFIRS) [2]. NFIRS is a reporting system used by fire departments nationwide to report on their activities. The system is maintained by the US Department of Homeland Security through the United States Fire Administration and is designed to capture all activities engaged in by a fire department, including fires, EMS and community outreach. The system is voluntary at the national level, so not all departments use the system or contribute data to it. Data is obtainable from the United State Fire Administration. Department information was from the National Fire Department registry maintained by the US Fire Administration [3].

The NFIRS system records the time, date, and street address of all incidents, the type of the incident (e.g., fire, EMS call, hazardous materials incident, service call, etc.), property use, equipment and personnel on the call, number type and severity of casualties, actions take, and a host of other data. For fires specifically, NFIRS collects information on the size of the fire, room of origin, heat source, item first ignited, human and other factors contributing to ignition, presence and effectiveness of detectors and automatic suppression equipment among other data. Note, that this data is generated through firefighter data entry. The quality of this information is directly dependent on firefighters accurately entering data.

Fires and EMS calls were geocoded as part of the FireCARES project. Street addresses entered into NFIRS are not always validated, so geocoding was not $100 \%$ effective. Geocoding percentage varied from department to department.

Department size classifications used in this work are shown in Table 1. Size classification is based on the classifications contained in the National Fire Protection Association (NFPA) Standard 1710 [2].

Demographic data was at the census tract level and was from the US Census' American Community Survey (ACS). "The $[\mathrm{ACS}]$ is a nationwide survey designed to provide communities with reliable and timely social, economic, housing, and demographic data every year.... The ACS has an annual sample size of about 3.5 million addresses.... Data are pooled across a calendar year to produce estimates for that year. As a result, ACS estimates reflect data that have been collected over a period of time rather than for a single point in time... [2]" This work uses the ACS 5-year estimates which are based on data pooled over a 5-year period.

Health information used in EMS estimation is from the Behavioral Risk Factor Surveillance System (BRFSS). The BRFSS "is the nation's premier system of health-related telephone surveys that collect state data about U.S. residents regarding their health-related risk behaviors, chronic health conditions, and use of preventive services.... The BRFSS completes more than 400000 adult interviews each year, making it the largest continuously conducted health survey system in the world. [3]" It is conducted by the 50 US States and compiled by US Centers for Disease Control (CDC).

As used in this report, the BFRSS data was drawn from two intermediate sources. County-level summaries of the BRFSS data were obtained from Robert Woods Johnson Foundation at

Table 1: Size groups for departments

\begin{tabular}{rrr} 
Size & \multicolumn{2}{r}{ Population Protected } \\
Group & Minimum & Maximum \\
\hline 0 & 0 & 2499 \\
1 & 2500 & 4999 \\
2 & 5000 & 9999 \\
3 & 10000 & 24999 \\
4 & 25000 & 49999 \\
5 & 50000 & 99999 \\
6 & 100000 & 249999 \\
7 & 250000 & 499999 \\
8 & 500000 & 999999 \\
9 & 1000000 & no max \\
\hline
\end{tabular}


www.countyhealthdata.org [7]. Census-tract level estimates of the BRFSS data were obtained from the 500-cities data set [5]. The 500-cities uses the BRFSS data and the techniques of small-area estimation [6] to estimate the values of the BRFSS survey questions at the census tract level for 500 U.S. cities.

A Social Vulnerability Index developed by the CDC is included. It is intended to be an aggregate index that represents the decree of vulnerability that a community has to disasters and hazards based on its social and demographic characteristics. [7]

For high risk and medium risk properties, some information at the Assessors' Parcel level were used. Parcel data were from CoreLogic [11], which aggregates assessors' parcel information for the entire country.

Information on smoking percentages at the state level are summarized from Census' National Health Interview Survey (NHIS) which has been conducted since 1957. "The NHIS is a large-scale household interview survey of a statistically representative sample of the U.S. civilian noninstitutionalized population. Interviewers visit 35000 to 40000 households across the country and collect data about 75000 to 100000 individuals. [8]"

Each census tract was associated with a specific department as part of this estimation. In a number of cases, the NFIRS data reported multiple departments responding to fires in a single census tract. There were a number of reasons for this. In some cases, this was a simple product of a geolocation error. In other cases, it was a mutual aid call. Some census tracts are served by multiple departments. If NFIRS reported multiple departments responding to a particular tract, then the department responding to the most calls for that tract became the "owner" of the tract. In case of a tie, then the department with the department id first in alphabetical order was selected. Ties occurred in only about $2 \%$ of cases.

Specific fields used in the analyses below, descriptions and their individual sources are listed in Appendix A.

Data from 2007 to 2013 was used to estimate the models.

Census tracts were filtered using the following filters:

- base

Tracts with any of the following characteristics were excluded:

○ $\quad$ SVI $<0$

- No reported median income

- No reported department size

$\circ$ No reported County smoking data 
- $\quad$ small.x

Departments (and tracts) are excluded if the department responded to fewer incidents than a specified floor, or if the number of incidents it responded to was an outlier compared to other years for the same department. The objective is to exclude years where reporting issues are a significant factor in the data. For low-hazard properties the definition of small varied depending on the model. Specifics of the various small filters are listed in Table 2. For all other risk models the 'small.0a' definition was used. The use of a variety of definitions for lowhazard properties made it possible to evaluate the effectiveness of the various definitions.

"Outlier" years were defined as those years which fell at least 2 standard deviations below the mean number of incidents for the department. Note that the "two standard deviations" standard is based on at most 7 years per department of data reported as part of the study.

- giants

Any tracts in the top $0.1 \%$ for population, number of housing units, number of males, or people in the age range of 45 to 54 were excluded from model estimation. This was done because direct examination of the data indicated that many of these tracts were not linearly related to the rest of the data set and tended to have disproportionate influence on the model.

- random_subset

One third of the tracts was selected at random and set aside to serve as a test set. The remaining tracts are used as the training set.

The total number of records for each property hazard group and the percent of records removed by each filter is listed in Table 3.

\section{Department Size $\times$ Region}

All departments serving fewer than 10000 people (sizes 0 to 2 in Table 1 above) are excluded from the analysis. All departments nationwide serving one million or more people were analyzed together. For some models (more details below) departments in the Northeast in size group 8 (see Table 1) were combined with those in the size group 7.

Table 3: Percent of records removed for each filter by property hazard level.

\begin{tabular}{lrrrrr} 
& \multicolumn{1}{c}{ N } & Total & base & small & giants \\
Low Hazard & 963001 & $60 \%$ & $59 \%$ & $39 \%$ & $1 \%$ \\
Medium Hazard & 963001 & $61 \%$ & $60 \%$ & $39 \%$ & \\
High Hazard & 1845431 & $49 \%$ & $48 \%$ & $29 \%$ & \\
EMS: 500-cities & 296393 & $80 \%$ & $80 \%$ & $5 \%$ & \\
EMS: county & 296393 & $70 \%$ & $70 \%$ & $5 \%$ &
\end{tabular}




\subsection{Models}

All models except those for high-hazard properties are estimated at the census tract level. High-hazard properties are estimated at the Assessors' parcel level.

Models fell into three broad categories: linear models, usually with department random effects; Random Forest models; and LASSO (least absolute shrinkage and selection operator) models.

All the linear random effects models were estimated using the techniques of generalized linear models (GLM), and had the following basic form:

$$
g\left(E\left(y_{i j t}\right)\right)=a_{i j t}+t \beta_{t}+x_{i j t}^{\prime} \beta+\eta_{j}
$$

Where $\mathrm{E}$ is the expectation operator, $y_{i j t}$ is the dependent variable being analyzed for the $i$ th tract served by the $j$ th department in year $t, g$ is a linking function used in the glm analysis, $a_{i j t}$ is a known offset value for the model, $x_{i j t}$ are the predictors used for that observation, $\beta_{t}$ is the change per year, $\beta$ are the parameter estimates, $\eta_{j}$ is the department effect.

It is further assumed that

$$
\eta_{j} \sim N\left(0, \sigma_{D}^{2}\right),
$$

where $\sigma_{D}^{2}$ is the variance of the department effects, and is estimated as part of the model. What is reported in the model is $\sigma_{D}^{2}$. Specific values for the individual departments can be provided on request. The one exception to this was the models for high-hazard properties, which did not include department random effects.

It was assumed that the number of fires, EMS calls, injuries or deaths in a tract follow a (over-dispersed) Poisson process. An offset term is used to reflect the proportion of incidents that were geolocated by department. That should convert these estimates, based on geolocated EMS calls, fires, injuries or deaths, into a model of total EMS calls, fires, injuries or deaths.

It was assumed that fire size followed a binomial model. No offset was used for the fire size models because the number of relevant fires was already included as part of the model.

In Random Forest models [9], a small random sample is drawn from the data and a regression tree is fit to it. This is repeated a large number of times, and the results of the random trees is averaged for the result. For this to work, all variables were included in the random forest estimation except for the department random effects. For medium-hazard properties separate models with 500 and 2500 trees were grown. Since the difference between the two was minimal, all other models used only 500 trees for the random forest.

LASSO models [9] estimate a standard linear model, but with the addition of a penalty term on the magnitude of the parameters. The penalty is the sum of the absolute values of the predictors. This results in parameters that are smaller than they would be in a regular linear regression. In fact, due to the form of the LASSO model some parameters will have a value of exactly zero. A LASSO model was run for fires, fire deaths and injuries, and EMS calls. The offset term was included as part of the LASSO analysis, and all the variables listed above were included. A large number of LASSO models were run and compared using cross-validation over the training set. Two LASSO models were selected for evaluation against the test set: the model with the cross-validation minimum error ("min"), and the model with the largest cross-validated error within 1 standard error of the minimum ("1se").

In addition, three naïve models were run for each analysis: a constant model, a pure department effects model, and a "tract" model. The constant model fits a single constant to the data (and does not include 
department effects). In effect, it assumes that all tracts have the same (average) expected value per year. The department-effect model fits the department effects only to the data. In effect, it assumes that all tracts served by a particular department have the same expected value per year. The "tract" model predicts that the value for a tract would be the same as the value for that tract experienced in the previous year. Since the dataset did not include 2006, no estimate was made for 2007 for the tract model.

Geocoding is incomplete and varies by department and year. All models (except for the fire-size models) are adjusted for geocoding percentage at the department $\times$ year level, by including the geocoding percentage of all reported incidents (by department and year) as an offset to the model.

\subsection{Analysis Groups}

Several separate analyses are conducted as part of this work to estimate community risk. Fires (and firerelated quantities) are analyzed separately based on fire hazard types as defined in NFPA Standard 1710 [10]. In the standard, low-hazard properties consist of one-, two- or three-family dwellings and scattered small business and industrial occupancies. Medium hazard properties consist of apartments, offices, mercantile and industrial occupancies not normally requiring extensive rescue by firefighting forces. High-hazard properties consist of schools, hospitals, nursing homes, explosive plants, refineries, highrise buildings and other high life hazard or large fire potential occupancies. There is a low-hazard occupancy fire-risk estimate, a medium hazard occupancy fire-risk estimate, etc. For each hazard type, fire risk is estimated as well as injury and death risk from fire, estimates of the proportion of fires that grow beyond the room of origin, and estimates of the proportion of fires that grow beyond the structure of origin.

A separate analysis is conducted of EMS risk.

The discussion below is structured based on property hazard levels (for fires and fire-related quantities) and EMS calls. 


\section{Fire Risk in Low-Hazard Properties}

In this section a series of models are presented that model community risk levels for number of fires, fire size, and number of fire injuries and deaths in low-hazard properties.

Predictors used in the model were organized into subgroups to simplify model selection for most models. The groups are summarized in Table 4 (see Appendix A for definitions of the variables).

A large number of different linear models were run, each with different combinations of the groups of predictor variables listed above. The models fall into two main classes. In the first group (labeled ' $L$ '), separate models were estimated for each region $\times$ department size group of departments (while excluding the 'region' group from the analysis). In the second group (labeled ' $S$ '), all models were estimated over the entire data set while including the 'region' dummies.

\section{Results}

All models are estimated against the training set. Then the estimated model is used to predict number of fires (or percent of fires for fire size, or fire injuries or deaths, depending on the model) for each tract in the test set. Then for each model the Root Mean Square error (RMSE) of the predicted value is calculated for the test set. Note that all models (except the "constant" model, the random forest model, and the LASSO model) reported here include a department dummy as part of the model estimate. All "Dummies" models also include dummies for department size and region, in addition to the variables listed. There are a few tracts in the test set associated with departments which do not appear in the training set and, therefore, for which no department dummy could be estimated. In those cases, the department dummy for that department was arbitrarily assigned a value of zero, effectively assuming that it behaves like the "average" department.

In all cases below, the test set was restricted to departments serving 100000 people or more. Since much of the model testing for low-hazard properties involved different definitions of small, a test set was needed that used a criterion different from the various small filters. The restriction based on department size was deemed the best way of determining what effect the various small filters had on predictions for the departments that will most likely be using the FireCARES system. Note that the test set against

Table 4:Predictor groups used for low-hazard property models.

\begin{tabular}{|c|c|c|}
\hline Group & Terms & Source \\
\hline Time & Year, with 2014 being zero & NFIRS \\
\hline Base & population, number of males, and hse_units & ACS \\
\hline Race & black, amer_es, other, hispanic, & ACS \\
\hline Age & $\begin{array}{l}\text { age_under5, age_5_9, age_10_14, age_15_19, age_20_24, } \\
\text { age_35_44, age_45_54, age_55_64, age_65_74, age_75_84, } \\
\text { age_85_up }\end{array}$ & ACS \\
\hline House & vacant, renter_occ, crowded, sfr,units_10, mh, older & ACS \\
\hline Personal & inc_hh, svi, married, unemployed, nilf, smoke_st, smoke_cty & ACS \\
\hline Fuel & $\begin{array}{l}\text { fuel_gas, fuel_tank, fuel_oil, fuel_coal, fuel_wood, fuel_solar, } \\
\text { fuel_other, fuel_none }\end{array}$ & ACS \\
\hline Region & region, fd_size, & FireCARES \\
\hline Department & fd_id & FireCARES \\
\hline
\end{tabular}


which RMSE was computed stayed the same regardless of the definition of the small filter used. That enabled an "apples to apples" comparison when assessing the effectiveness of the various models.

Results are reported in Appendix B. Comparing the results to the averages per tract in the test set indicates that the models for fires, fire injuries and deaths are still over-dispersed relative to a Poisson model. Overfitting of the models is apparent for many models estimating fire size, fire injuries or deaths. It is a severe problem for deaths.

For the most part, the best models compare well to the naïve models. As a rule, about half the reduction in variance is attributable to the department effect. The "tract" naïve model beats the other model for fires, but does poorly for all the other models. Remember that the tract ID contains a lot of information, including department ID and all the census data. That is why it performs so well against the fire models. However, once the event being predicted becomes rare (e.g., $88 \%$ of the time there are zero injuries in a tract), then the tract label contains only a little information about occurrence. So, pooling information about similar tracts, which is essentially what the models do, improves the prediction. 


\section{Fire Risk in Medium-Hazard Properties}

In this section a series of models are estimated that model community risk levels for number of fires in medium-hazard properties, fire size, and number of fire injuries and deaths for medium-hazard properties.

As with low-hazard properties, predictors used in the model were organized into subgroups to simplify model selection for most models. The groups are summarized in Table 5.

A large number of different linear models were run, each with different combinations of the groups of predictor variables listed above. The models fall into two main classes. In the first group, separate models for each region $\times$ department size group of departments were estimated (while excluding the 'region' group from the analysis). In the second group, all models were estimated over the entire data set while including the 'region' dummies.

\section{Results}

All models are estimated against the training set. Then the estimated model is used to predict the number of fires (or percent of fires for fire size, or fire injuries or deaths, depending on the model) for each tract in the test set. Then for each model the RMSE of the predicted value is calculated for the test set. Note that all models except the constant, LASSO and random forest models, include a department dummy as part of the model estimate. All "Dummies" models, and the LASSO and random forest models also include dummies for department size and region, in addition to the variables listed. There are a few tracts in the test set associated with departments which do not appear in the training set and, therefore, for which no department dummy could be estimated. In those cases, the department dummy for that department was arbitrarily assigned a value of zero.

In all cases below, the test set is restricted to departments serving 100000 people or more. This is consistent with the test set used in the low-hazard property models.

Results are reported in Appendix C. Comparing the results to the averages per tract in the test set indicates that the models for fires, fire injuries and deaths are still over-dispersed relative to a Poisson

Table 5:Predictor groups used for medium-hazard property models.

\begin{tabular}{|c|c|c|}
\hline Group & Terms & Source \\
\hline Time & Year, with 2014 being zero & NFIRS \\
\hline Base & pop, males, and hse_units & ACS \\
\hline Race & black, amer_es, other, hispanic, & ACS \\
\hline Age & $\begin{array}{l}\text { age_under5, age_5_9, age_10_14, age_15_19, age_20_24, } \\
\text { age_35_44, age_45_54, age_55_64, age_65_74, age_75_84, } \\
\text { age_85_up }\end{array}$ & ACS \\
\hline House & $\begin{array}{l}\text { vacant, renter_occ, crowded, sfr, units_10, mh, older, apt_parcels, } \\
\text { mr_parcels }\end{array}$ & $\begin{array}{l}\text { ACS, } \\
\text { CoreLogic }\end{array}$ \\
\hline Personal & inc_hh, svi, married, unemployed, nilf, smoke_st, smoke_cty & $\mathrm{ACS}$ \\
\hline Fuel & $\begin{array}{l}\text { fuel_gas, fuel_tank, fuel_oil, fuel_coal, fuel_wood, fuel_solar, } \\
\text { fuel_other, fuel_none }\end{array}$ & $\mathrm{ACS}$ \\
\hline Region & region, fd_size, & FireCARES \\
\hline Department & fd_id & FireCARES \\
\hline
\end{tabular}


model. Overfitting of the models is apparent for many models estimating number of fires, fire size, fire injuries or deaths.

For fires, the random forest model handily beat all other models except for the "tract" model. That is particularly impressive considering that the random forest models do not include department effects. The "tract" naïve model did very well for fires, but poorly for all the other effects. As with low risk fires, the tract ID contains a lot of information, including department ID and all the census data, but for fire injuries and deaths contains little information about occurrence.

For fire deaths, the LASSO model is the best predictor. 


\section{Fire Risk in High-Hazard Properties}

In this section a series of models are estimated that model community risk levels for number of fires in high-hazard properties, fire size, and number of fire injuries and deaths for high-hazard properties.

Unlike in the previous analyses, this analysis operates at the parcel level. Predictions are rolled up to the census tract level for FireCARES.

Predictors used in the model were organized into subgroups to simplify model selection for most models. The groups are summarized in Table 6 .

Occupancy class was included in the set of predictors because the risks presented by the different classes of properties were expected to be different. Occupancy class was included in all models. The remaining groups where included in some models but not all.

\section{Data}

Risk for high-hazard properties was determined differently from the determination for low and medium hazard properties. Risk was estimated at the parcel level.

Parcels were selected from the CoreLogic data set based on the specific properties identified by the FireCARES team from other data sets. Fires were identified based on their geocoded location. Any fire that overlapped the bounding box for a high-risk parcel and was a structural fire (any NFIRS incident type beginning with ' 11 ') was included as a high-risk fire and associated with the overlapping parcel. Note that the bounding box is guaranteed to be at least as large as the parcel, and in most cases will be bigger.

There are several potential problems with the incident selection approach. First it seems likely that some incidents at high-hazard properties were not included due to poor quality geocoding. Second, it seems likely that some non-high-hazard incidents were included, both due to geocoding issues and due to the fact that bounding boxes are often bigger than the actual parcels. Since high hazard parcels are a small percentage of the total number of parcels in the data set, the first effect seems likely to have a bigger effect than the latter.

Table 6:Predictor groups used for high-hazard property models.

\begin{tabular}{|c|c|c|}
\hline Group & Terms & Source \\
\hline Time & Year, with 2014 being zero & NFIRS \\
\hline Base & pop, males, and hse_units & ACS \\
\hline Race & black, amer_es, other, hispanic, & ACS \\
\hline Age & $\begin{array}{l}\text { age_under5, age_5_9, age_10_14, age_15_19, age_20_24, } \\
\text { age_35_44, age_45_54, age_55_64, age_65_74, age_75_84, } \\
\text { age_85_up }\end{array}$ & ACS \\
\hline House & vacant, renter_occ, crowded, sfr, units_10, mh, older & ACS \\
\hline Personal & inc_hh, svi, married, unemployed, nilf, smoke_st, smoke_cty & ACS \\
\hline $\begin{array}{l}\text { Occupancy } \\
\text { Class }\end{array}$ & occ_class & CoreLogic \\
\hline High Rise & hr_floors & CoreLogic \\
\hline Region & region, fd_size, & FireCARES \\
\hline Department & fd_id & FireCARES \\
\hline
\end{tabular}


Third, there are likely cases where the same incident is associated with more than one high-hazard parcel due to the fact that bounding boxes are (in some cases) larger than the parcels themselves. This problem was addressed by 'unioning' any overlapping high-hazard parcels into a single parcel group. Thus, if multiple parcels are in the same location, they are combined into a single parcel group, and then the parcel groups are matched to fires.

Since the approach used to identify high hazard parcels is different from that used to identify low and medium hazard parcels, the possibility exists of parcels being identified as both as high and medium or low hazard, and of parcels that end up unclassified. Table 7 lists the number of parcels in the CoreLogic data set by both their CoreLogic categorization and by their FireCARES categorization. Italicized groups are either double-counted or excluded.

As with the other classes of fires, geocoding is incomplete, and varies by department and year. This model adjusts for geocoding percentage at the department $\times$ year level, by including the geocoding percentage of all reported incidents (by department and year) as an offset to the model.

Different from the other property hazard groups, a department effect was not included.

\section{Results}

All models are estimated against the training set. Then the estimated model is used to predict number of fires (or percent of fires for fire size, or fire injuries or deaths, depending on the model) for each parcel in the test set. Then for each model the RMSE) of the predicted value is calculated for the test set. All "Dummies" models, and the LASSO and random forest models also include dummies for department size and region, in addition to the variables listed.

Since department effect was not included in this model, no pure-department effect naïve model was estimated. Instead a pure risk-class naïve model was estimated. It assumes that all tracts in the same risk-class have the same expected value per year.

In all cases below, the test set is restricted to departments serving 100000 people or more. This is consistent with the test set used in the low-hazard property models.

Results are reported in Appendix D. Overfitting is a noticeable problem for fire deaths and injuries. The random forest model is the best for everything except injuries. For injuries the best model is likely the model labeled "hr.0101011" for the "separate" class of models. However, there are many models that are very similar to that model and are likely indistinguishable from it. 


\section{EMS "Risk"}

In this section a series of models are estimated that model community risk levels EMS calls.

For EMS risk, two separate groups of models were run, depending on whether health information is from the 500-cities project or from countyhealthrankings.org. Since the 500-cities project provides data estimated at the census tract level, it is better suited to this analysis, but it does not cover the entire country. While the countyhealthrankings.org data is only at the county level, it is available for the entire country. The intent is to use the estimates based on the 500-cities data for those portions of the country where it is available, and to use estimates based on the countyhealthrankings.org data for the rest of the country.

\section{County Clusters}

The County Health Data and the ACS were summarized by county and clustered into nine clusters using the pam algorithm, part of the cluster package [11] in R [12].

All the data from the ACS and countyhealthrankings.org [4] used in the EMS analysis was used here for clustering by county. ACS Data was rolled up to the county level where necessary. For most columns, that amounted to summing up the column values for all tracts in a county. In a few cases (e.g., number of people per household), a weighted average was computed. All columns were standardized by mean and standard deviation before estimation of the clusters.

One cluster was dropped because it had no departments with EMS data. Each cluster represents a set of counties that are more similar to each other than they are to counties in other clusters. Figure 1 shows a map of the clustered counties.

As with the fire risk models, there were several different groups of linear models. The first group (labeled "S" for "Short") estimated a single model against the entire data set. This version always included the "regional" group of terms. The second group (labeled "L" for "Long") estimated separate models against each region $\times$ department-size group. Details are discussed in Section 2.1 above. The third group (labeled "C" for "Cluster" was unique to EMS risk) estimated separate models for each cluster.

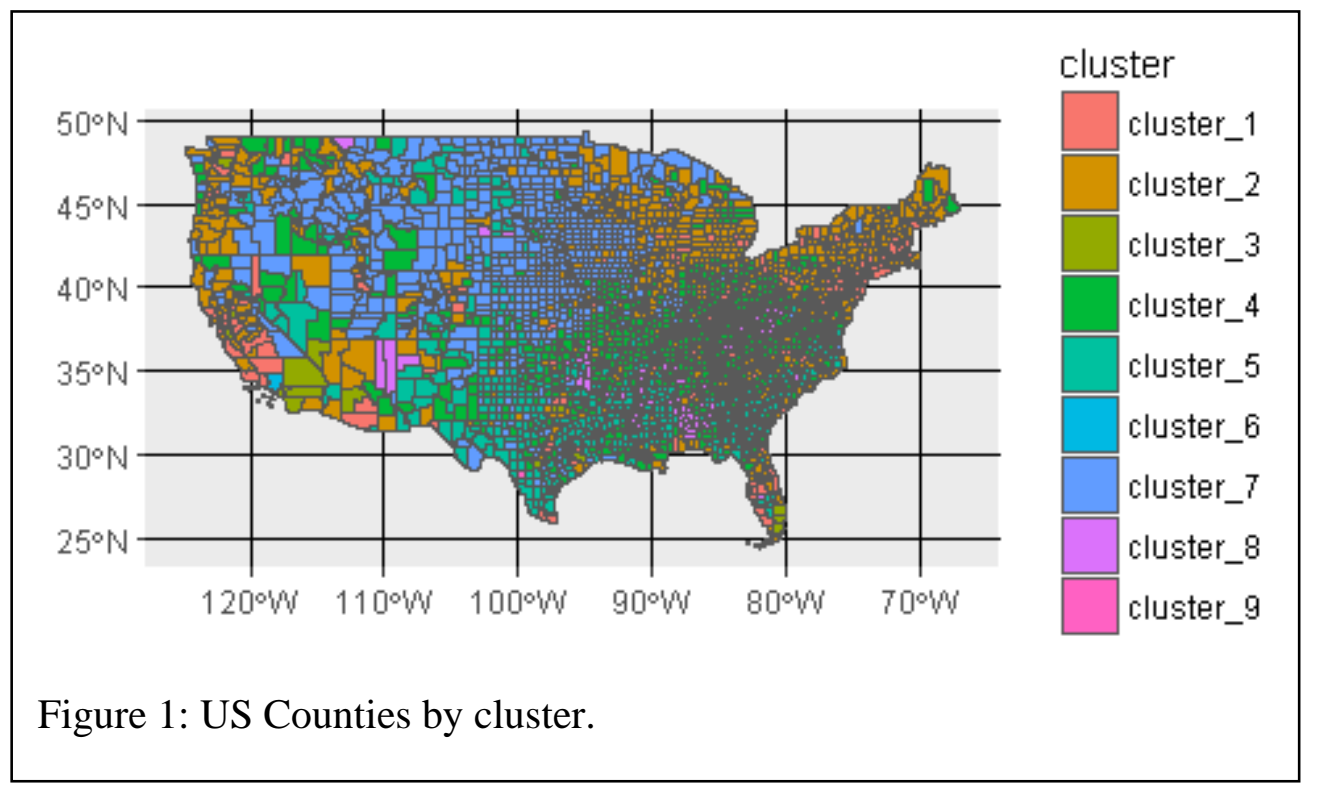


Table 8: Predictor groups used for the '500-cities' EMS models.

\begin{tabular}{lll} 
Group & Terms & Source \\
\hline Year & year & NFIRS \\
Base & ave_hh_sz, pop,males & ACS \\
Demographic & black, amer_es, other, hispanic, age_under5, age_5_9, age_10_14, & ACS \\
& age_15_19, age_20_24, age_35_44, age_45_54, age_55_64, & \\
age_65_74, age_75_84, age_85_up & \\
Housing & hse_units, vacant, renter_occ, crowded, sfr,units_10, mh,older & ACS \\
Personal & inc_hh, svi, married, unemployed, nilf & ACS \\
Fuel & fuel_gas, fuel_tank, fuel_oil, fuel_coal, fuel_wood, fuel_solar, & ACS \\
fuel_other, fuel_none & \\
Health1a & arthritis, bphigh, cancer, casthma, chd,copd, diabetes, highchol, & 500-Cities \\
kidney, mhlth, phlth, stroke, teethlost & \\
Health2a & $\begin{array}{l}\text { access2, binge, csmoking, lpa, obesity, sleep, bpmed, checkup, } \\
\text { cholscreen, colon_screen, corem, corew, dental, mammouse, paptest }\end{array}$ & 500-Cities \\
Degion & $\begin{array}{l}\text { region, fd_size, cluster } \\
\text { fc_dept_id }\end{array}$ & FireCARES
\end{tabular}

Predictors used in the model were organized into subgroups to simplify model selection for most models. The groups are summarized in Table 8 and Table 9.

\section{Analysis}

Results are listed in Appendix E.

A number of models fail for some subgroups. The typical reason for a model to fail is that some of the explanatory variables are collinear. This problem occurs in two cases. In one case, the subgroup analyzed is so small that estimation fails for even a moderate number of variables. The second case arises only for the county set of models. For the county models, the health-related information is the same for all tracts in a county. However, for some subgroups there are so few counties that even a moderate number of health-related variables present problems. That applies even though there may be thousands of tracts to work with.

There is very little that can be done for the first case. Since those subgroups inherently have few departments, tracts, EMS calls and people, those cases are ignored. The second case potentially presents serious problems, since some of those subgroups have large populations.

In Appendix E, any model that runs into problems of the second type are identified. Any model with those problems is not considered in identifying the best models for subsequent analysis.

There were no problem models in the 500-cities data set. The best model in that set was ems.5.124S with an RMSE of 362.9.

There were a large number of problem models in the county-based data set. After removing those from consideration, the best model in that set was ems.C.031C with an RMSE of 329.0. 
Table 9: Predictor groups used for the 'County' EMS models.

\begin{tabular}{lll} 
Group & Terms & Source \\
\hline Year & year & NFIRS \\
Base & ave_hh_sz, pop,males & ACS \\
Demographic & $\begin{array}{l}\text { Black, amer_es, other, hispanic, age_under5, age_5_9, age_10_14, } \\
\text { age_15_19, age_20_24, age_35_44, age_45_54, age_55_64, }\end{array}$ & \\
& age_65_74, age_75_84, age_85_up & \\
Housing & hse_units, vacant, renter_occ, crowded, sfr, units_10, mh, older & ACS \\
Personal & inc_hh, svi, married, unemployed, nilf & ACS \\
Fuel & $\begin{array}{l}\text { fuel_gas, fuel_tank, fuel_oil, fuel_coal, fuel_wood, fuel_solar, } \\
\text { fuel_other, fuel_none }\end{array}$ & ACS \\
Health1b & $\begin{array}{l}\text { diabetes, years_lost, poor_health, days_pr_hlth, days_pr_mntl, } \\
\text { low_birthwt, early_mortality, child_mortality, infant_death, hiv }\end{array}$ & RWJ \\
Health2b & $\begin{array}{l}\text { binge, csmoking, lpa, obesity, food_ndx, exercise_place, dui, stds, } \\
\text { teen_births, drug_overdose, mv_deaths, lack_sleep }\end{array}$ & RWJ \\
Health3b & $\begin{array}{l}\text { access2, physicians, dentists, shrinks, wrong_hosp, diabetic_scrn, } \\
\text { mammography, nurses, pm10, house_probs, drive_alone, }\end{array}$ & RWJ \\
& $\begin{array}{l}\text { long_commute, high_school, college, child_pov, inequality, } \\
\text { child_sngl_prnt, social, violent, injury_dths, food_insecurity, }\end{array}$ & \\
& $\begin{array}{l}\text { no_healthy_food, uninsured_adult, uninsured_child, hlth_cost, } \\
\text { free_lunch, segregation1, segregation2, homicide, rural }\end{array}$ & \\
region, fd_size, cluster & FireCARES \\
Region & fc_dept_id & FireCARES \\
Department
\end{tabular}




\section{Conclusions}

This report documents the methods used to estimate community risk levels for the FireCARES project. Community risk levels were estimated for fires, fire injuries and fire deaths for low- medium- and highhazard properties. Risks were estimated at the census tract level. In addition, community risk levels were estimated for EMS services.

The best models, and the models selected for production risk estimation, are listed in Table 10.

In most cases the model with the lowest RMS error was used. In a few cases a different model was selected. Where the difference in RMS errors was small, models with fewer predictors were preferred (and in particular " $\mathrm{S}$ " type models, i.e., models where the entire sample was estimated as a single model with dummies for department size and region were preferred over "L" type models). Models with time in the set of predictors were preferred to those without. Since random forest shows up as the model with the lowest RMS error for six out of the 17 test cases (and there were a number of other cases where it had an RMS error very close to the best) random forest was preferred to linear models.

With those considerations, the production models for fire size for low-hazard properties were used because they were more parsimonious than the base models. The production models for fires in medium hazard properties were used because they included time and the lowest RMS error models did not. The fire injury model for high-hazard properties was chosen because it was an "S" type model and the lowest RMS Error model was not. Random forest was chosen for the EMS "county" model because it had an RMS error very close to the lowest RMS error.

Table 10: Best models and models selected for FireCARES production use.

\begin{tabular}{|c|c|c|c|c|c|}
\hline \multirow[b]{2}{*}{ Model Group } & \multirow[b]{2}{*}{ Measure } & \multicolumn{2}{|c|}{ Lowest RMS Error } & \multicolumn{2}{|c|}{ Production Model } \\
\hline & & Model & RMS Error & Model & RMS Error \\
\hline Low Hazard & Fire & rForest & 2.3859 & \multicolumn{2}{|c|}{$\langle$ same $>$} \\
\hline Low Hazard & ‘Medium’ Fires & base.S & 0.3895 & M100111.S & 0.3895 \\
\hline Low Hazard & 'Large Fires & base.S & 0.2697 & M100111.S & 0.2697 \\
\hline Low Hazard & Fire Injury & M111110.S & 0.6254 & \multicolumn{2}{|c|}{ <same> } \\
\hline Low Hazard & Fire Death & LASSO & 0.1768 & \multicolumn{2}{|c|}{ <same> } \\
\hline Medium Hazard & Fire & rf.0500 & 2.9066 & \multicolumn{2}{|c|}{ <same> } \\
\hline Medium Hazard & ‘Medium’ Fires & mr.011110.S & 0.4064 & mr.111110.S & 0.4066 \\
\hline Medium Hazard & 'Large Fires & mr.101110.S & 0.3020 & \multicolumn{2}{|c|}{ <same> } \\
\hline Medium Hazard & Fire Injury & mr.000101.L & 0.3754 & mr.100101.L & 0.3755 \\
\hline Medium Hazard & Fire Death & LASSO & 0.0831 & \multicolumn{2}{|c|}{ <same> } \\
\hline High Hazard & Fire & rForest & 0.6190 & \multicolumn{2}{|c|}{$<$ same $>$} \\
\hline High Hazard & ‘Medium’ Fires & rForest & 0.1597 & \multicolumn{2}{|c|}{$<$ same $>$} \\
\hline High Hazard & 'Large Fires & rForest & 0.1948 & \multicolumn{2}{|c|}{$<$ same> } \\
\hline High Hazard & Fire Injury & hr.0101011.L & 0.1341 & hr.1110111S & 0.1352 \\
\hline High Hazard & Fire Death & rForest & 0.0092 & \multicolumn{2}{|c|}{ <same> } \\
\hline EMS & 500-Cities & ems.5.124.S & 362.9 & \multicolumn{2}{|c|}{ <same> } \\
\hline EMS & County & ems.C.031.C & 329.0 & rForest & 330.1 \\
\hline
\end{tabular}




\section{Works Cited}

[1] S. Gilbert and D. Butry, "Identifying Vulnerable Populations to Death and Injuries from Residential Fires," National Institute of Standards and Technology, 2016.

[2] US Census, Understanding and Using American Community Survey Data: What All Data Users Need to Know, US Census, 2018.

[3] CDC, "CDC - BRFSS," 2018. [Online]. Available: https://www.cdc.gov/brfss/index.html. [Accessed 179 2018].

[4] Robert Wood Johnson Foundation, "County Health Rankings," [Online]. Available: www.countyhealthrankings.org.

[5] X. Zhang, J. Holt, H. Lu, A. Wheaton, E. Ford, K. Greenlund and J. Croft, "Multilevel Regression and Poststratification for Small-Area Estimation of Population Health Outcomes: A Case Study of Chronic Obstructive Pulmonary Disease Prevalence Using the Behavioral Risk Factor Surveillance System," American Journal of Epidemiology, vol. 179, no. 8, pp. 1025-1033, 2014.

[6] J. Rao and I. Molina, Small Area Estimation, Hoboken, New Jersey: John Wiley \& Sons, Inc, 2015.

[7] B. Flanagan, E. Gregory, E. Hallisey, J. Heitgerd and B. Lewis, "A Social Vulnerability Index for Disaster Management," Journal of Homeland Security and Emergency Management, vol. 8, no. 1, 2011.

[8] US Department of Health and Human Services, National Health Interview Survey, US Department of Health and Human Services, 2018.

[9] T. Hastie, R. Tibshirani and J. Friedman, The Elements of Statistical Learning, Springer Science and Business Media, 2009.

[10] National Fire Protection Association, "Standard for the Organization and Deployment of Fire Suppression Operations, Emergency Medical Operations, and Special Operations to the Public by Career Fire Departments," NFPA, 2016.

[11] M. Maechler, P. Rousseeuw, A. Struyf, M. Hubert and K. Hornik, "Cluster: Cluster Anaysis Basics and Extensions," 2018.

[12] R Core Team, "R: A Language and Environment for Statistical Computing," R Foundation for Statistical Computing, Vienna, Austria, 2018. 


\section{Appendix A: Variables and Data Sources}

\begin{tabular}{|c|c|c|}
\hline Predictor & Description & Source \\
\hline year & year, with 2014 as zero & NFIRS \\
\hline region & Census Region & NFIRS \\
\hline fd_id & Department ID & NFIRS \\
\hline fd_size & Department Size (See Section 2.1) & NFIRS \\
\hline cluster & Cluster (see Section 6) & \\
\hline f_located & proportion incidents geolocated & NFIRS \\
\hline c_located & proportion ems incidents geolocated & NFIRS \\
\hline ave_hh_sz & Average household size & ACS \\
\hline pop & Population & ACS \\
\hline black & Number of black people in census tract & ACS \\
\hline amer_es & Number of american indian/eskimo people in census tract & ACS \\
\hline other & Number of people whose race is not white, black or amer_es & ACS \\
\hline hispanic & Number of hispanics & ACS \\
\hline males & Number of males & ACS \\
\hline age_under5 & Number of people whose age is $<5$ & ACS \\
\hline age_5_9 & Number of people whose age is between 5 and 9 & ACS \\
\hline age_10_14 & Number of people whose age is between 10 and 14 & ACS \\
\hline age_15_19 & Number of people whose age is between 15 and 19 & ACS \\
\hline age_20_24 & Number of people whose age is between 20 and 24 & ACS \\
\hline age_25_34 & Number of people whose age is between 25 and 34 & ACS \\
\hline age_35_44 & Number of people whose age is between 35 and 44 & ACS \\
\hline age_45_54 & Number of people whose age is between 45 and 54 & ACS \\
\hline age_55_64 & Number of people whose age is between 55 and 64 & ACS \\
\hline age_65_74 & Number of people whose age is between 65 and 74 & ACS \\
\hline age_75_84 & Number of people whose age is between 75 and 84 & ACS \\
\hline age_85_up & Number of people whose age is 85 or higher & ACS \\
\hline hse_units & Number of housing units & ACS \\
\hline vacant & Number of housing units vacant & ACS \\
\hline renter_occ & Number of renter-occupied housing units & ACS \\
\hline crowded & Number of housing unit with more people than rooms & ACS \\
\hline sfr & Number of single-family residences & ACS \\
\hline units_10 & Number of housing units part of a 10 -unit or larger complex & ACS \\
\hline $\mathrm{mh}$ & Number of mobile homes & ACS \\
\hline older & Number of homes built before 1980 & ACS \\
\hline apt_parcels & Number of Assessors parcels zoned for apartments & CoreLogic \\
\hline mr_parcels & Number of assessors parcels zoned commercial & CoreLogic \\
\hline inc_hh & Average household income & ACS \\
\hline svi & Social Vulnerability Index & ACS \\
\hline married & Number of married people & ACS \\
\hline unemployed & Number of unemployed & ACS \\
\hline nilf & Number of people older than 15 and not in labor force & ACS \\
\hline smoke_st & $\%$ of smokers in state & NHIS \\
\hline smoke_cty & $\%$ of smokers in the county & county health \\
\hline
\end{tabular}




Predictor
fuel_gas
fuel_tank
fuel_oil
fuel_coal
fuel_wood
fuel_solar
fuel_other
fuel_none
bld_units
hr_floors
eff_year
risk_class
arthritis
bphigh
cancer
casthma
chd
copd
diabetes
highchol
kidney
mhlth
phlth
stroke
teethlost
access2
bpmed
checkup
cholscreen
colon_screen
corem
corew
dental
mammouse
paptest
Ipange
obesity
sleep

years_lost

\section{Description}

Number of households using gas for heating

Number of households using tanked gas for heating

Number of households using oil for heating

Number of households using coal for heating

Number of households using wood for heating

Number of households using solar for heating

Number of households using another fuel for heating

Number of households without heating

Number of building units on a parcel

Number of floors

Year built

One of "Assembly," "High Rise," "Industrial," "Institutional,"
"Medical," and "School."

$\%$ of adults with arthritis

$\%$ of adults with high blood pressure

$\%$ of adults who have ever had cancer

$\%$ of adults who currently have asthma

$\%$ of adults with coronary heart disease

$\%$ of adults with Chronic obstructive pulmonary disease

$\%$ of adults with diabetes

$\%$ of adults with high cholesterol

$\%$ of adults with chronic kidney disease

$\%$ of adults with poor mental health for $>=14$ of the last 30 days

$\%$ of adults with poor health for $>=14$ of the last 30 days

$\%$ of adults who have had a stroke

$\%$ of adults who have lost all their teeth

$\%$ adult uninsured

$\%$ of those prescribed who are taking blood pressure meds

$\%$ of adults who have had a checkup in the last year

$\%$ of adults who have had cholesterol screening in the last 5 years

$\%$ of adults $50-75$ who have had colon exam

$\%$ of men $>=65$ who have had a set of core medical services

$\%$ of women $>=65$ who have had a set of core medical services

$\%$ of adults who have been to the dentist in the last year

$\%$ of women 40-75 who have had a mammogram in the last 2 years

$\%$ of women 21-65 who have had a pap smear in the last 3 years

$\%$ of adults "binge" drinking in the last 30 days

$\%$ of adults who are current smokers

$\%$ of adults with no leisure physical activity in the last month

$\%$ of adults who are obese

$\%$ of adults averaging $<7$ hours of sleep per night

Estimated years of potential life lost before age 75
Source

ACS

ACS

ACS

ACS

ACS

ACS

ACS

ACS

CoreLogic

CoreLogic

CoreLogic

CoreLogic

500-Cities

500-Cities

500-Cities

500-Cities

500-Cities

500-Cities

500-Cities

500-Cities

500-Cities

500-Cities

500-Cities

500-Cities

500-Cities

500-Cities

500-Cities

500-Cities

500-Cities

500-Cities

500-Cities

500-Cities

500-Cities

500-Cities

500-Cities

500-Cities

500-Cities

500-Cities

500-Cities

500-Cities

county health 


\begin{tabular}{|c|c|c|}
\hline Predictor & Description & Source \\
\hline poor_health & $\%$ of adults with poor health for $>=14$ of the last 30 days & county health \\
\hline days_pr_hlth & days of poor health for $>=14$ of the last 30 days & county health \\
\hline days_pr_mntl & days of poor mental health for $>=14$ of the last 30 days & county health \\
\hline low_birthwt & $\%$ of live births with low birthweight & county health \\
\hline csmoking & $\%$ of adults who are current smokers & county health \\
\hline obesity & $\%$ of adults who are obese & county health \\
\hline food_ndx & Food environment index & county health \\
\hline Ipa & $\%$ of adults with no leisure physical activity in the last month & county health \\
\hline exercise_place & Access to exercise opportunities & county health \\
\hline binge & $\%$ of adults "binge" drinking in the last 30 days & county health \\
\hline dui & \# alcohol-impaired driving deaths & county health \\
\hline stds & Chlamydia cases per 100000 & county health \\
\hline teen_births & Teen birth rate & county health \\
\hline access 2 & $\%$ adult uninsured & county health \\
\hline physicians & Primary care physicians per 100000 & county health \\
\hline dentists & Dentists per 100000 & county health \\
\hline shrinks & Mental health providers per 100000 & county health \\
\hline wrong_hosp & Preventable hospital stay rate & county health \\
\hline diabetic_scrn & $\%$ of diabetics with testing & county health \\
\hline mammography & $\begin{array}{l}\% \text { of women } 40-75 \text { who have had a mammogram in the last } 2 \\
\text { years }\end{array}$ & county health \\
\hline high_school & $\%$ of adults with high-school diploma & county health \\
\hline college & $\%$ of adults with some college & county health \\
\hline child_pov & \# of children in poverty & county health \\
\hline inequality & Income inequality & county health \\
\hline child_sngl_prnt & \# of children in single-parent households & county health \\
\hline social & social associations & county health \\
\hline violent & \# of violent crimes & county health \\
\hline injury_dths & \# of injury deaths & county health \\
\hline pm10 & air pollution & county health \\
\hline house_probs & severe housing problems & county health \\
\hline drive_alone & $\%$ of commuters who drive alone to work & county health \\
\hline long_commute & $\%$ of commuters with a long commute driving alone & county health \\
\hline early_mortality & Premature age-adjsted mortality & county health \\
\hline child_mortality & Child mortality rate & county health \\
\hline infant_death & Infant mortality rate & county health \\
\hline phys_distress & $\%$ frequent physical distress & county health \\
\hline mntl_distress & $\%$ frequent mental distress & county health \\
\hline diabetes & diabetes prevalence & county health \\
\hline hiv & HIV prevalence & county health \\
\hline food_insecurity & $\%$ Food insecurity & county health \\
\hline no_healthy_food & $\%$ with limited access to healthy foods & county health \\
\hline drug_overdose & Drug overdose death rate & county health \\
\hline mv_deaths & Moter vehicle accident death rate & county health \\
\hline
\end{tabular}




\begin{tabular}{|c|c|}
\hline Predictor & Description \\
\hline lack_sleep & $\%$ insufficent sleep \\
\hline uninsured_adult & $\%$ adults uninsured \\
\hline uninsured_child & $\%$ children uninsured \\
\hline hlth_cost & Health care costs \\
\hline nurses & Other primary care provider rate \\
\hline free_lunch & Children eligible for free lunch \\
\hline segregation 1 & black/while segregation \\
\hline segregation2 & white/non-white segregation \\
\hline homicide & Homicides \\
\hline rural & $\%$ rural \\
\hline
\end{tabular}

Source

county health

county health

county health

county health

county health

county health

county health

county health

county health

county health 


\section{Appendix B: Low- Hazard Properties: Model Results}

Table 11: Root Mean Square (RMS) Errors of models of fire risk for low hazard properties.

Model(s) with the lowest RMS Error are in bold. A blank field indicates that the relevant model was not run.

\begin{tabular}{|c|c|c|c|c|c|c|}
\hline \multirow[b]{2}{*}{ Model Run } & \multirow[b]{2}{*}{ Predictors } & \multicolumn{2}{|c|}{ Small Filter } & \multirow[b]{2}{*}{ Mean } & \multicolumn{2}{|c|}{ MSE } \\
\hline & & Floor & Outlier & & Separate & Dummies \\
\hline Constant & & 25 & $\mathrm{X}$ & 2.1228 & & 2.9559 \\
\hline dept.effect & & 25 & $\mathrm{X}$ & 2.1228 & & 2.8447 \\
\hline tract & & & & 2.1228 & & 2.5570 \\
\hline lasso.min & time, base, race, age, house, personal, fuel & 25 & $\mathrm{X}$ & 2.1228 & & 2.6180 \\
\hline lasso.1se & time, base, race, age, house, personal, fuel & 25 & $\mathrm{X}$ & 2.1228 & & 2.6526 \\
\hline rForest & time, base, race, age, house, personal, fuel & 25 & $\mathrm{X}$ & 2.1228 & & 2.3859 \\
\hline base & time, base, race, age, house, personal, fuel & 25 & $\mathrm{X}$ & 2.1228 & 2.6641 & 2.6191 \\
\hline M101111 & time, base, age, house, personal, fuel & 25 & $\mathrm{X}$ & 2.1228 & 2.6383 & 2.6244 \\
\hline M100111 & time, base, house, personal, fuel & 25 & $\mathrm{X}$ & 2.1228 & 2.6046 & 2.6176 \\
\hline M101011 & time, base, age, personal, fuel & 25 & $\mathrm{X}$ & 2.1228 & 2.7534 & 2.7888 \\
\hline M101101 & time, base, age, house, fuel & 25 & $\mathrm{X}$ & 2.1228 & 2.6115 & 2.6290 \\
\hline M101110 & time, base, age, house, personal & 25 & $\mathrm{X}$ & 2.1228 & 2.6392 & 2.6186 \\
\hline M110111 & time, base, race, house, personal, fuel & 25 & $\mathrm{X}$ & 2.1228 & 2.6322 & 2.6271 \\
\hline M110011 & time, base, race, personal, fuel & 25 & $\mathrm{X}$ & 2.1228 & 2.7421 & 2.7856 \\
\hline M110101 & time, base, race, house, fuel & 25 & $X$ & 2.1228 & 2.6474 & 2.6435 \\
\hline M110110 & time, base, race, house, personal & 25 & $\mathrm{X}$ & 2.1228 & 2.6353 & 2.6189 \\
\hline M111011 & time, base, race, age, personal, fuel & 25 & $X$ & 2.1228 & 2.7735 & 2.7504 \\
\hline M111001 & time, base, race, age, fuel & 25 & $\mathrm{X}$ & 2.1228 & 2.8366 & 2.8048 \\
\hline M111010 & time, base, race, age, personal & 25 & $\mathrm{X}$ & 2.1228 & 2.7798 & 2.7541 \\
\hline M111101 & time, base, race, age, house, fuel & 25 & $\mathrm{X}$ & 2.1228 & 2.6579 & 2.6322 \\
\hline M111100 & time, base, race, age, house & 25 & $\mathrm{X}$ & 2.1228 & 2.6626 & 2.6242 \\
\hline M111110 & time, base, race, age, house, personal & 25 & $\mathrm{X}$ & 2.1228 & 2.6635 & 2.6130 \\
\hline f.050 & time, base, house, personal, fuel & 50 & $\mathrm{X}$ & 2.1228 & 2.6055 & \\
\hline f.100 & time, base, house, personal, fuel & 100 & $\mathrm{X}$ & 2.1228 & 2.6060 & \\
\hline f. 150 & time, base, house, personal, fuel & 150 & $\mathrm{X}$ & 2.1228 & 2.6083 & \\
\hline f. 200 & time, base, house, personal, fuel & 200 & $\mathrm{X}$ & 2.1228 & 2.6119 & \\
\hline f. 250 & time, base, house, personal, fuel & 250 & $\mathrm{X}$ & 2.1228 & 2.6260 & \\
\hline f.500 & time, base, house, personal, fuel & 500 & $X$ & 2.1228 & 2.6378 & \\
\hline f.000 & time, base, house, personal, fuel & 1000 & $\mathrm{X}$ & 2.1228 & 2.9886 & \\
\hline
\end{tabular}


Table 12: RMS Errors of models of percent of fires that grow beyond the room of origin for low hazard properties.

Model(s) with the lowest RMS Error are in bold. A blank field indicates that the relevant model was not run.

\begin{tabular}{|c|c|c|c|c|c|c|}
\hline \multirow[b]{2}{*}{ Model Run } & \multirow[b]{2}{*}{ Predictors } & \multicolumn{2}{|c|}{ Small Filter } & \multirow{2}{*}{$\begin{array}{c}\text { Mean } \\
(\%)\end{array}$} & \multicolumn{2}{|c|}{ MSE } \\
\hline & & Floor & Outlier & & Separate & Dummies \\
\hline Constant & & 25 & $\mathrm{X}$ & 0.4247 & 0.4 & 132 \\
\hline dept.effect & & 25 & $\mathrm{X}$ & 0.4247 & 0.3 & 909 \\
\hline tract & & & & 0.4247 & 0.5 & 995 \\
\hline base & time, base, race, age, house, personal, fuel & 25 & $X$ & 0.4247 & 0.3901 & 0.3895 \\
\hline M101111 & time, base, age, house, personal, fuel & 25 & $\mathrm{X}$ & 0.4247 & 0.3908 & 0.3896 \\
\hline M100111 & time, base, house, personal, fuel & 25 & $\mathrm{X}$ & 0.4247 & 0.3901 & 0.3895 \\
\hline M101011 & time, base, age, personal, fuel & 25 & $X$ & 0.4247 & 0.3910 & 0.3899 \\
\hline M101101 & time, base, age, house, fuel & 25 & $\mathrm{X}$ & 0.4247 & 0.3909 & 0.3898 \\
\hline M101110 & time, base, age, house, personal & 25 & $X$ & 0.4247 & 0.3908 & 0.3897 \\
\hline M110111 & time, base, race, house, personal, fuel & 25 & $X$ & 0.4247 & 0.3901 & 0.3895 \\
\hline M110011 & time, base, race, personal, fuel & 25 & $X$ & 0.4247 & 0.3903 & 0.3900 \\
\hline M110101 & time, base, race, house, fuel & 25 & $X$ & 0.4247 & 0.3901 & 0.3897 \\
\hline M110110 & time, base, race, house, personal & 25 & $\mathrm{X}$ & 0.4247 & 0.3901 & 0.3896 \\
\hline M111011 & time, base, race, age, personal, fuel & 25 & $\mathrm{X}$ & 0.4247 & 0.3910 & 0.3899 \\
\hline M111001 & time, base, race, age, fuel & 25 & $X$ & 0.4247 & 0.3911 & 0.3903 \\
\hline M111010 & time, base, race, age, personal & 25 & $\mathrm{X}$ & 0.4247 & 0.3911 & 0.3902 \\
\hline M111101 & time, base, race, age, house, fuel & 25 & $X$ & 0.4247 & 0.3909 & 0.3898 \\
\hline M111100 & time, base, race, age, house & 25 & $\mathrm{X}$ & 0.4247 & 0.3909 & 0.3899 \\
\hline M111110 & time, base, race, age, house, personal & 25 & $\mathrm{X}$ & 0.4247 & 0.3909 & 0.3896 \\
\hline
\end{tabular}


Table 13: RMS Errors of models of percent of fires that grow beyond the structure of origin for low hazard properties.

Model(s) with the lowest RMS Error are in bold. A blank field indicates that the relevant model was not run.

\begin{tabular}{|c|c|c|c|c|c|c|}
\hline \multirow[b]{2}{*}{ Model Run } & \multirow[b]{2}{*}{ Predictors } & \multicolumn{2}{|c|}{ Small Filter } & \multirow{2}{*}{$\begin{array}{c}\text { Mean } \\
(\%)\end{array}$} & \multicolumn{2}{|c|}{ MSE } \\
\hline & & Floor & Outlier & & Separate & Dummies \\
\hline Constant & & 25 & X & 0.1082 & & \\
\hline dept.effect & & 25 & $\mathrm{X}$ & 0.1082 & & \\
\hline tract & & & & 0.1082 & & \\
\hline base & time, base, race, age, house, personal, fuel & 25 & $\mathrm{X}$ & 0.1082 & 0.2708 & 0.2697 \\
\hline M101111 & time, base, age, house, personal, fuel & 25 & $\mathrm{X}$ & 0.1082 & 0.2722 & 0.2698 \\
\hline M100111 & time, base, house, personal, fuel & 25 & $\mathrm{X}$ & 0.1082 & 0.2708 & 0.2697 \\
\hline M101011 & time, base, age, personal, fuel & 25 & $\mathrm{X}$ & 0.1082 & 0.2717 & 0.2699 \\
\hline M101101 & time, base, age, house, fuel & 25 & $\mathrm{X}$ & 0.1082 & 0.2716 & 0.2697 \\
\hline M101110 & time, base, age, house, personal & 25 & $\mathrm{X}$ & 0.1082 & 0.2722 & 0.2702 \\
\hline M110111 & time, base, race, house, personal, fuel & 25 & $\mathrm{X}$ & 0.1082 & 0.2706 & 0.2698 \\
\hline M110011 & time, base, race, personal, fuel & 25 & $\mathrm{X}$ & 0.1082 & 0.2702 & 0.2699 \\
\hline M110101 & time, base, race, house, fuel & 25 & $\mathrm{X}$ & 0.1082 & 0.2705 & 0.2698 \\
\hline M110110 & time, base, race, house, personal & 25 & $\mathrm{X}$ & 0.1082 & 0.2708 & 0.2703 \\
\hline M111011 & time, base, race, age, personal, fuel & 25 & $\mathrm{X}$ & 0.1082 & 0.2715 & 0.2699 \\
\hline M111001 & time, base, race, age, fuel & 25 & $\mathrm{X}$ & 0.1082 & 0.2710 & 0.2700 \\
\hline M111010 & time, base, race, age, personal & 25 & $\mathrm{X}$ & 0.1082 & 0.2715 & 0.2705 \\
\hline M111101 & time, base, race, age, house, fuel & 25 & $\mathrm{X}$ & 0.1082 & 0.2715 & 0.2699 \\
\hline M111100 & time, base, race, age, house & 25 & $\mathrm{X}$ & 0.1082 & 0.2715 & 0.2703 \\
\hline M111110 & time, base, race, age, house, personal & 25 & $\mathrm{X}$ & 0.1082 & 0.2719 & 0.2703 \\
\hline
\end{tabular}


Table 14: RMS Errors of models of fire injury for low hazard properties.

Model(s) with the lowest RMS Error are in bold. A blank field indicates that the relevant model was not run.

\begin{tabular}{|c|c|c|c|c|c|c|}
\hline \multirow[b]{2}{*}{ Model Run } & \multirow[b]{2}{*}{ Predictors } & \multicolumn{2}{|c|}{ Small Filter } & \multirow[b]{2}{*}{ Mean } & \multicolumn{2}{|c|}{ MSE } \\
\hline & & Floor & Outlier & & Separate & ummies \\
\hline Constant & & 25 & X & 0.1805 & & \\
\hline dept.effect & & 25 & $\mathrm{X}$ & 0.1805 & & \\
\hline tract & & & & 0.1805 & & \\
\hline lasso.min & time, base, race, age, house, personal, fuel & 25 & $\mathrm{X}$ & 0.1805 & & \\
\hline lasso.1se & time, base, race, age, house, personal, fuel & 25 & $\mathrm{X}$ & 0.1805 & & \\
\hline rForest & time, base, race, age, house, personal, fuel & 25 & $\mathrm{X}$ & 0.1805 & & \\
\hline base & time, base, race, age, house, personal, fuel & 25 & $\mathrm{X}$ & 0.1805 & 0.6448 & 0.6263 \\
\hline M101111 & time, base, age, house, personal, fuel & 25 & $\mathrm{X}$ & 0.1805 & 0.6479 & 0.6257 \\
\hline M100111 & time, base, house, personal, fuel & 25 & $\mathrm{X}$ & 0.1805 & 0.6448 & 0.6263 \\
\hline M101011 & time, base, age, personal, fuel & 25 & $\mathrm{X}$ & 0.1805 & 0.6333 & 0.6260 \\
\hline M101101 & time, base, age, house, fuel & 25 & $\mathrm{X}$ & 0.1805 & 0.6469 & 0.6273 \\
\hline M101110 & time, base, age, house, personal & 25 & $\mathrm{X}$ & 0.1805 & 0.6449 & 0.6258 \\
\hline M110111 & time, base, race, house, personal, fuel & 25 & $\mathrm{X}$ & 0.1805 & 0.6478 & 0.6264 \\
\hline M110011 & time, base, race, personal, fuel & 25 & $\mathrm{X}$ & 0.1805 & 0.6313 & 0.6275 \\
\hline M110101 & time, base, race, house, fuel & 25 & $\mathrm{X}$ & 0.1805 & 0.6478 & 0.6281 \\
\hline M110110 & time, base, race, house, personal & 25 & $\mathrm{X}$ & 0.1805 & 0.6415 & 0.6264 \\
\hline M111011 & time, base, race, age, personal, fuel & 25 & $\mathrm{X}$ & 0.1805 & 0.6395 & 0.6260 \\
\hline M111001 & time, base, race, age, fuel & 25 & $\mathrm{X}$ & 0.1805 & 0.6411 & 0.6313 \\
\hline M111010 & time, base, race, age, personal & 25 & $\mathrm{X}$ & 0.1805 & 0.6361 & 0.6261 \\
\hline M111101 & time, base, race, age, house, fuel & 25 & $\mathrm{X}$ & 0.1805 & 0.6505 & 0.6276 \\
\hline M111100 & time, base, race, age, house & 25 & $\mathrm{X}$ & 0.1805 & 0.6469 & 0.6275 \\
\hline M111110 & time, base, race, age, house, personal & 25 & $\mathrm{X}$ & 0.1805 & 0.6475 & 0.6254 \\
\hline
\end{tabular}


Table 15: RMS Errors of models of fire deaths for low hazard properties.

Model(s) with the lowest RMS Error are in bold. A blank field indicates that the relevant model was not run.

\begin{tabular}{|c|c|c|c|c|c|c|}
\hline \multirow[b]{2}{*}{ Model Run } & \multirow[b]{2}{*}{ Predictors } & \multicolumn{2}{|c|}{ Small Filter } & \multirow[b]{2}{*}{ Mean } & \multicolumn{2}{|c|}{ MSE } \\
\hline & & Floor & Outlier & & Separate & ummies \\
\hline Constant & & 25 & X & 0.0184 & & \\
\hline dept.effect & & 25 & $\mathrm{X}$ & 0.0184 & & \\
\hline tract & & & & 0.0184 & & \\
\hline lasso.min & time, base, race, age, house, personal, fuel & 25 & $\mathrm{X}$ & 0.0184 & & \\
\hline lasso.1se & time, base, race, age, house, personal, fuel & 25 & $\mathrm{X}$ & 0.0184 & & \\
\hline rForest & time, base, race, age, house, personal, fuel & 25 & $\mathrm{X}$ & 0.0184 & & \\
\hline base & time, base, race, age, house, personal, fuel & 25 & $\mathrm{X}$ & 0.0184 & 5.3604 & 0.1769 \\
\hline M101111 & time, base, age, house, personal, fuel & 25 & $\mathrm{X}$ & 0.0184 & 6.3266 & 0.1769 \\
\hline M100111 & time, base, house, personal, fuel & 25 & $\mathrm{X}$ & 0.0184 & 5.3604 & 0.1769 \\
\hline M101011 & time, base, age, personal, fuel & 25 & $\mathrm{X}$ & 0.0184 & 0.1793 & 0.1769 \\
\hline M101101 & time, base, age, house, fuel & 25 & $\mathrm{X}$ & 0.0184 & 2.5591 & 0.1769 \\
\hline M101110 & time, base, age, house, personal & 25 & $\mathrm{X}$ & 0.0184 & 4.0317 & 0.1769 \\
\hline M110111 & time, base, race, house, personal, fuel & 25 & $\mathrm{X}$ & 0.0184 & 7.0284 & 0.1769 \\
\hline M110011 & time, base, race, personal, fuel & 25 & $\mathrm{X}$ & 0.0184 & 0.1778 & 0.1770 \\
\hline M110101 & time, base, race, house, fuel & 25 & $\mathrm{X}$ & 0.0184 & 2.9679 & 0.1769 \\
\hline M110110 & time, base, race, house, personal & 25 & $\mathrm{X}$ & 0.0184 & 2.9177 & 0.1769 \\
\hline M111011 & time, base, race, age, personal, fuel & 25 & $\mathrm{X}$ & 0.0184 & 0.1794 & 0.1769 \\
\hline M111001 & time, base, race, age, fuel & 25 & $\mathrm{X}$ & 0.0184 & 0.1784 & 0.1770 \\
\hline M111010 & time, base, race, age, personal & 25 & $\mathrm{X}$ & 0.0184 & 0.1780 & 0.1770 \\
\hline M111101 & time, base, race, age, house, fuel & 25 & $\mathrm{X}$ & 0.0184 & 3.5768 & 0.1769 \\
\hline M111100 & time, base, race, age, house & 25 & $\mathrm{X}$ & 0.0184 & 2.2835 & 0.1769 \\
\hline M111110 & time, base, race, age, house, personal & 25 & $\mathrm{X}$ & 0.0184 & 5.5102 & 0.1769 \\
\hline
\end{tabular}




\section{Appendix C: Medium- Hazard Properties: Model Results}

Table 16: RMS Errors of models of fire risk for medium hazard properties.

Model(s) with the lowest RMS Error are in bold. A blank field indicates that the relevant model was not run.

\begin{tabular}{|c|c|c|c|c|}
\hline Model Run & Predictors & Mean & \multicolumn{2}{|c|}{$\begin{array}{l}\text { RMSE } \\
\text { rate Dummies }\end{array}$} \\
\hline constant & & 1.96 & \multicolumn{2}{|c|}{4.2898} \\
\hline fx & & 1.96 & \multicolumn{2}{|c|}{3.8521} \\
\hline tract & & 1.96 & \multicolumn{2}{|c|}{2.9349} \\
\hline lasso.min & time, base, race, age, house, personal & 1.96 & \multicolumn{2}{|c|}{5.4329} \\
\hline lasso.1se & time, base, race, age, house, personal & 1.96 & \multicolumn{2}{|c|}{5.1042} \\
\hline rf.0500 & time, base, race, age, house, personal & 1.96 & \multicolumn{2}{|c|}{2.9066} \\
\hline rf. 2500 & time, base, race, age, house, personal & 1.96 & \multicolumn{2}{|c|}{2.9066} \\
\hline mr.100000 & time & 1.96 & 3.8528 & 3.8550 \\
\hline mr.010000 & base & 1.96 & 6.0926 & 4.0019 \\
\hline mr. 110000 & time, base & 1.96 & 6.0855 & 4.0033 \\
\hline mr.001000 & race & 1.96 & 9.1421 & 7.4384 \\
\hline mr.101000 & time, race & 1.96 & 9.1411 & 7.4365 \\
\hline mr.011000 & base, race & 1.96 & 14.0619 & 18.1033 \\
\hline mr.111000 & time, base, race & 1.96 & 14.0684 & 18.0627 \\
\hline mr.000100 & age & 1.96 & 3.5807 & 3.6102 \\
\hline mr.100100 & time, age & 1.96 & 3.5794 & 3.6134 \\
\hline mr.010100 & base, age & 1.96 & 4.0325 & 8.3532 \\
\hline mr.110100 & time, base, age & 1.96 & 4.0801 & 8.7797 \\
\hline mr.001100 & race, age & 1.96 & 4.9517 & 6.2661 \\
\hline mr.101100 & time, race, age & 1.96 & 4.9333 & 6.2540 \\
\hline mr.011100 & base, race, age & 1.96 & 4.8144 & 13.8594 \\
\hline mr.111100 & time, base, race, age & 1.96 & 4.7710 & 14.3395 \\
\hline mr.000010 & house & 1.96 & 3.6017 & 4.1742 \\
\hline mr.100010 & time, house & 1.96 & 3.6040 & 4.1763 \\
\hline mr.010010 & base, house & 1.96 & 3.5931 & 4.3357 \\
\hline mr.110010 & time, base, house & 1.96 & 3.5861 & 4.3348 \\
\hline mr.001010 & race, house & 1.96 & 4.5524 & 5.4363 \\
\hline mr.101010 & time, race, house & 1.96 & 4.5539 & 5.4312 \\
\hline mr.011010 & base, race, house & 1.96 & 4.5188 & 5.9286 \\
\hline mr.111010 & time, base, race, house & 1.96 & 4.5158 & 5.9245 \\
\hline mr.000110 & age, house & 1.96 & 3.6150 & 4.1887 \\
\hline mr.100110 & time, age, house & 1.96 & 3.6181 & 4.1992 \\
\hline mr.010110 & base, age, house & 1.96 & 3.6307 & 3.8707 \\
\hline mr.110110 & time, base, age, house & 1.96 & 3.6364 & 3.8759 \\
\hline mr.001110 & race, age, house & 1.96 & 3.7425 & 4.4266 \\
\hline mr.101110 & time, race, age, house & 1.96 & 3.7372 & 4.4276 \\
\hline mr.011110 & base, race, age, house & 1.96 & 4.4387 & 5.2162 \\
\hline mr.111110 & time, base, race, age, house & 1.96 & 4.4181 & 5.1980 \\
\hline mr.000001 & personal & 1.96 & 5.3904 & 4.3304 \\
\hline
\end{tabular}




\begin{tabular}{|c|c|c|c|c|}
\hline \multirow{2}{*}{$\begin{array}{l}\text { Model Run } \\
\text { mr.100001 }\end{array}$} & Predictors & Mean & \multicolumn{2}{|c|}{\begin{tabular}{|c} 
RMSE \\
Separate Dummies
\end{tabular}} \\
\hline & time, personal & 1.96 & 5.3992 & 4.3367 \\
\hline mr.010001 & base, personal & 1.96 & 3.9126 & 4.7478 \\
\hline mr.110001 & time, base, personal & 1.96 & 3.9665 & 4.7706 \\
\hline mr.001001 & race, personal & 1.96 & 7.5266 & 5.6002 \\
\hline mr.101001 & time, race, personal & 1.96 & 7.5237 & 5.6153 \\
\hline mr.011001 & base, race, personal & 1.96 & 4.3830 & 6.1931 \\
\hline mr.111001 & time, base, race, personal & 1.96 & 4.4337 & 6.2262 \\
\hline mr.000101 & age, personal & 1.96 & 3.9020 & 5.0191 \\
\hline mr.100101 & time, age, personal & 1.96 & 3.8976 & 5.0339 \\
\hline mr.010101 & base, age, personal & 1.96 & 3.7502 & 4.1935 \\
\hline mr.110101 & time, base, age, personal & 1.96 & 3.7622 & 4.1918 \\
\hline mr.001101 & race, age, personal & 1.96 & 4.2938 & 5.9742 \\
\hline mr.101101 & time, race, age, personal & 1.96 & 4.2904 & 5.9802 \\
\hline mr.011101 & base, race, age, personal & 1.96 & 4.1424 & 5.4903 \\
\hline mr.111101 & time, base, race, age, personal & 1.96 & 4.1526 & 5.4922 \\
\hline mr.000011 & house, personal & 1.96 & 3.4203 & 5.0597 \\
\hline mr.100011 & time, house, personal & 1.96 & 3.4223 & 5.0871 \\
\hline mr.010011 & base, house, personal & 1.96 & 3.7852 & 5.4390 \\
\hline mr.110011 & time, base, house, personal & 1.96 & 3.7756 & 5.4675 \\
\hline mr.001011 & race, house, personal & 1.96 & 4.0709 & 6.5501 \\
\hline mr.101011 & time, race, house, personal & 1.96 & 4.0924 & 6.5815 \\
\hline mr.011011 & base, race, house, personal & 1.96 & 4.8339 & 7.7235 \\
\hline mr.111011 & time, base, race, house, personal & 1.96 & 4.8298 & 7.7502 \\
\hline mr.000111 & age, house, personal & 1.96 & 3.6996 & 5.0109 \\
\hline mr.100111 & time, age, house, personal & 1.96 & 3.6901 & 5.0014 \\
\hline mr.010111 & base, age, house, personal & 1.96 & 4.1191 & 5.4157 \\
\hline mr.110111 & time, base, age, house, personal & 1.96 & 4.1015 & 5.3867 \\
\hline mr.001111 & race, age, house, personal & 1.96 & 4.1740 & 5.5691 \\
\hline mr.101111 & time, race, age, house, personal & 1.96 & 4.1772 & 5.5584 \\
\hline mr.011111 & base, race, age, house, personal & 1.96 & 4.9311 & 6.8501 \\
\hline mr.base & time, base, race, age, house, personal & 1.96 & 4.9303 & 6.8467 \\
\hline
\end{tabular}


Table 17: RMS Errors of models of percent of fires that grow beyond the room of origin for medium hazard properties.

Model(s) with the lowest RMS Error are in bold. A blank field indicates that the relevant model was not run.

\begin{tabular}{|c|c|c|c|c|}
\hline \multirow{5}{*}{$\begin{array}{l}\text { Model Run } \\
\text { constant } \\
\text { fx } \\
\text { tract } \\
\text { mr } 100000\end{array}$} & Predictors & Mean (\%) & \multicolumn{2}{|c|}{$\begin{array}{c}\text { RMSE } \\
\text { Separate Dummies }\end{array}$} \\
\hline & & 0.3653 & 0.4 & \\
\hline & & 0.3653 & 0.4 & \\
\hline & & 0.3653 & 0.5 & \\
\hline & time & 0.3653 & 0.4103 & 0.4100 \\
\hline mr.010000 & base & 0.3653 & 0.4089 & 0.4088 \\
\hline mr.110000 & time, base & 0.3653 & 0.4094 & 0.4090 \\
\hline mr.001000 & race & 0.3653 & 0.4095 & 0.4096 \\
\hline mr.101000 & time, race & 0.3653 & 0.4099 & 0.4098 \\
\hline mr.011000 & base, race & 0.3653 & 0.4084 & 0.4086 \\
\hline mr.111000 & time, base, race & 0.3653 & 0.4089 & 0.4088 \\
\hline mr.000100 & age & 0.3653 & 0.4090 & 0.4086 \\
\hline mr.100100 & time, age & 0.3653 & 0.4093 & 0.4087 \\
\hline mr.010100 & base, age & 0.3653 & 0.4085 & 0.4081 \\
\hline mr.110100 & time, base, age & 0.3653 & 0.4089 & 0.4083 \\
\hline mr.001100 & race, age & 0.3653 & 0.4086 & 0.4084 \\
\hline mr.101100 & time, race, age & 0.3653 & 0.4090 & 0.4086 \\
\hline mr.011100 & base, race, age & 0.3653 & 0.4082 & 0.4080 \\
\hline mr.111100 & time, base, race, age & 0.3653 & 0.4085 & 0.4082 \\
\hline mr.000010 & house & 0.3653 & 0.4066 & 0.4066 \\
\hline mr.100010 & time, house & 0.3653 & 0.4069 & 0.4067 \\
\hline mr.010010 & base, house & 0.3653 & 0.4065 & 0.4065 \\
\hline mr.110010 & time, base, house & 0.3653 & 0.4068 & 0.4067 \\
\hline mr.001010 & race, house & 0.3653 & 0.4064 & 0.4065 \\
\hline mr.101010 & time, race, house & 0.3653 & 0.4067 & 0.4067 \\
\hline mr.011010 & base, race, house & 0.3653 & 0.4064 & 0.4064 \\
\hline mr.111010 & time, base, race, house & 0.3653 & 0.4067 & 0.4066 \\
\hline mr.000110 & age, house & 0.3653 & 0.4072 & 0.4066 \\
\hline mr.100110 & time, age, house & 0.3653 & 0.4075 & 0.4068 \\
\hline mr.010110 & base, age, house & 0.3653 & 0.4070 & 0.4065 \\
\hline mr.110110 & time, base, age, house & 0.3653 & 0.4073 & 0.4066 \\
\hline mr.001110 & race, age, house & 0.3653 & 0.4070 & 0.4066 \\
\hline mr.101110 & time, race, age, house & 0.3653 & 0.4073 & 0.4067 \\
\hline mr.011110 & base, race, age, house & 0.3653 & 0.4068 & 0.4064 \\
\hline mr.111110 & time, base, race, age, house & 0.3653 & 0.4072 & 0.4066 \\
\hline mr.000001 & personal & 0.3653 & 0.4097 & 0.4098 \\
\hline mr.100001 & time, personal & 0.3653 & 0.4102 & 0.4100 \\
\hline mr.010001 & base, personal & 0.3653 & 0.4086 & 0.4084 \\
\hline mr.110001 & time, base, personal & 0.3653 & 0.4090 & 0.4086 \\
\hline mr.001001 & race, personal & 0.3653 & 0.4093 & 0.4096 \\
\hline mr.101001 & time, race, personal & 0.3653 & 0.4097 & 0.4098 \\
\hline
\end{tabular}




\begin{tabular}{|c|c|c|c|c|}
\hline Model Run & Predictors & Mean (\%) & $\begin{array}{r}\text { RN } \\
\text { Separate }\end{array}$ & $\begin{array}{l}\mathrm{E} \\
\text { Immies }\end{array}$ \\
\hline mr.011001 & base, race, personal & 0.3653 & 0.4083 & 0.4082 \\
\hline mr.111001 & time, base, race, personal & 0.3653 & 0.4087 & 0.4084 \\
\hline mr.000101 & age, personal & 0.3653 & 0.4090 & 0.4085 \\
\hline mr.100101 & time, age, personal & 0.3653 & 0.4093 & 0.4087 \\
\hline mr.010101 & base, age, personal & 0.3653 & 0.4085 & 0.4081 \\
\hline mr.110101 & time, base, age, personal & 0.3653 & 0.4089 & 0.4083 \\
\hline mr.001101 & race, age, personal & 0.3653 & 0.4087 & 0.4084 \\
\hline mr.101101 & time, race, age, personal & 0.3653 & 0.4091 & 0.4085 \\
\hline mr.011101 & base, race, age, personal & 0.3653 & 0.4084 & 0.4079 \\
\hline mr.111101 & time, base, race, age, personal & 0.3653 & 0.4088 & 0.4081 \\
\hline mr.000011 & house, personal & 0.3653 & 0.4069 & 0.4067 \\
\hline mr.100011 & time, house, personal & 0.3653 & 0.4073 & 0.4069 \\
\hline mr.010011 & base, house, personal & 0.3653 & 0.4068 & 0.4065 \\
\hline mr.110011 & time, base, house, personal & 0.3653 & 0.4071 & 0.4067 \\
\hline mr.001011 & race, house, personal & 0.3653 & 0.4069 & 0.4067 \\
\hline mr.101011 & time, race, house, personal & 0.3653 & 0.4072 & 0.4069 \\
\hline mr.011011 & base, race, house, personal & 0.3653 & 0.4068 & 0.4065 \\
\hline mr.111011 & time, base, race, house, personal & 0.3653 & 0.4072 & 0.4067 \\
\hline mr.000111 & age, house, personal & 0.3653 & 0.4075 & 0.4066 \\
\hline mr.100111 & time, age, house, personal & 0.3653 & 0.4078 & 0.4068 \\
\hline mr.010111 & base, age, house, personal & 0.3653 & 0.4072 & 0.4065 \\
\hline mr.110111 & time, base, age, house, personal & 0.3653 & 0.4076 & 0.4067 \\
\hline mr.001111 & race, age, house, personal & 0.3653 & 0.4074 & 0.4066 \\
\hline mr.101111 & time, race, age, house, personal & 0.3653 & 0.4077 & 0.4068 \\
\hline mr.011111 & base, race, age, house, personal & 0.3653 & 0.4073 & 0.4065 \\
\hline mr.base & time, base, race, age, house, personal & 0.3653 & 0.4076 & 0.4066 \\
\hline
\end{tabular}


Table 18: RMS Errors of models of percent of fires that grow beyond the structure of origin for medium hazard properties.

Model(s) with the lowest RMS Error are in bold. A blank field indicates that the relevant model was not run.

\begin{tabular}{|c|c|c|c|c|}
\hline \multirow{5}{*}{$\begin{array}{l}\text { Model Run } \\
\text { constant } \\
\text { fx } \\
\text { tract } \\
\text { mr.100000 }\end{array}$} & Predictors & Mean (\%) & \multicolumn{2}{|c|}{$\begin{array}{c}\text { RMSE } \\
\text { Separate Dummies }\end{array}$} \\
\hline & & 0.1229 & 0.3 & \\
\hline & & 0.1229 & 0.3 & \\
\hline & & 0.1229 & 0.3 & \\
\hline & time & 0.1229 & 0.3043 & 0.3040 \\
\hline mr.010000 & base & 0.1229 & 0.3046 & 0.3037 \\
\hline mr.110000 & time, base & 0.1229 & 0.3046 & 0.3036 \\
\hline mr.001000 & race & 0.1229 & 0.3047 & 0.3041 \\
\hline mr.101000 & time, race & 0.1229 & 0.3048 & 0.3041 \\
\hline mr.011000 & base, race & 0.1229 & 0.3049 & 0.3037 \\
\hline mr.111000 & time, base, race & 0.1229 & 0.3049 & 0.3036 \\
\hline mr.000100 & age & 0.1229 & 0.3060 & 0.3033 \\
\hline mr.100100 & time, age & 0.1229 & 0.3061 & 0.3033 \\
\hline mr.010100 & base, age & 0.1229 & 0.3065 & 0.3032 \\
\hline mr.110100 & time, base, age & 0.1229 & 0.3066 & 0.3031 \\
\hline mr.001100 & race, age & 0.1229 & 0.3059 & 0.3033 \\
\hline mr.101100 & time, race, age & 0.1229 & 0.3061 & 0.3033 \\
\hline mr.011100 & base, race, age & 0.1229 & 0.3068 & 0.3032 \\
\hline mr.111100 & time, base, race, age & 0.1229 & 0.3070 & 0.3031 \\
\hline mr.000010 & house & 0.1229 & 0.3044 & 0.3022 \\
\hline mr.100010 & time, house & 0.1229 & 0.3045 & 0.3021 \\
\hline mr.010010 & base, house & 0.1229 & 0.3052 & 0.3021 \\
\hline mr.110010 & time, base, house & 0.1229 & 0.3053 & 0.3021 \\
\hline mr.001010 & race, house & 0.1229 & 0.3050 & 0.3021 \\
\hline mr.101010 & time, race, house & 0.1229 & 0.3050 & 0.3021 \\
\hline mr.011010 & base, race, house & 0.1229 & 0.3054 & 0.3022 \\
\hline mr.111010 & time, base, race, house & 0.1229 & 0.3055 & 0.3021 \\
\hline mr.000110 & age, house & 0.1229 & 0.3071 & 0.3021 \\
\hline mr.100110 & time, age, house & 0.1229 & 0.3073 & 0.3020 \\
\hline mr.010110 & base, age, house & 0.1229 & 0.3074 & 0.3021 \\
\hline mr.110110 & time, base, age, house & 0.1229 & 0.3077 & 0.3020 \\
\hline mr.001110 & race, age, house & 0.1229 & 0.3071 & 0.3020 \\
\hline mr.101110 & time, race, age, house & 0.1229 & 0.3073 & 0.3020 \\
\hline mr.011110 & base, race, age, house & 0.1229 & 0.3075 & 0.3021 \\
\hline mr.111110 & time, base, race, age, house & 0.1229 & 0.3079 & 0.3020 \\
\hline mr.000001 & personal & 0.1229 & 0.3048 & 0.3037 \\
\hline mr.100001 & time, personal & 0.1229 & 0.3048 & 0.3036 \\
\hline mr.010001 & base, personal & 0.1229 & 0.3054 & 0.3031 \\
\hline mr.110001 & time, base, personal & 0.1229 & 0.3054 & 0.3031 \\
\hline mr.001001 & race, personal & 0.1229 & 0.3051 & 0.3036 \\
\hline mr.101001 & time, race, personal & 0.1229 & 0.3051 & 0.3036 \\
\hline
\end{tabular}




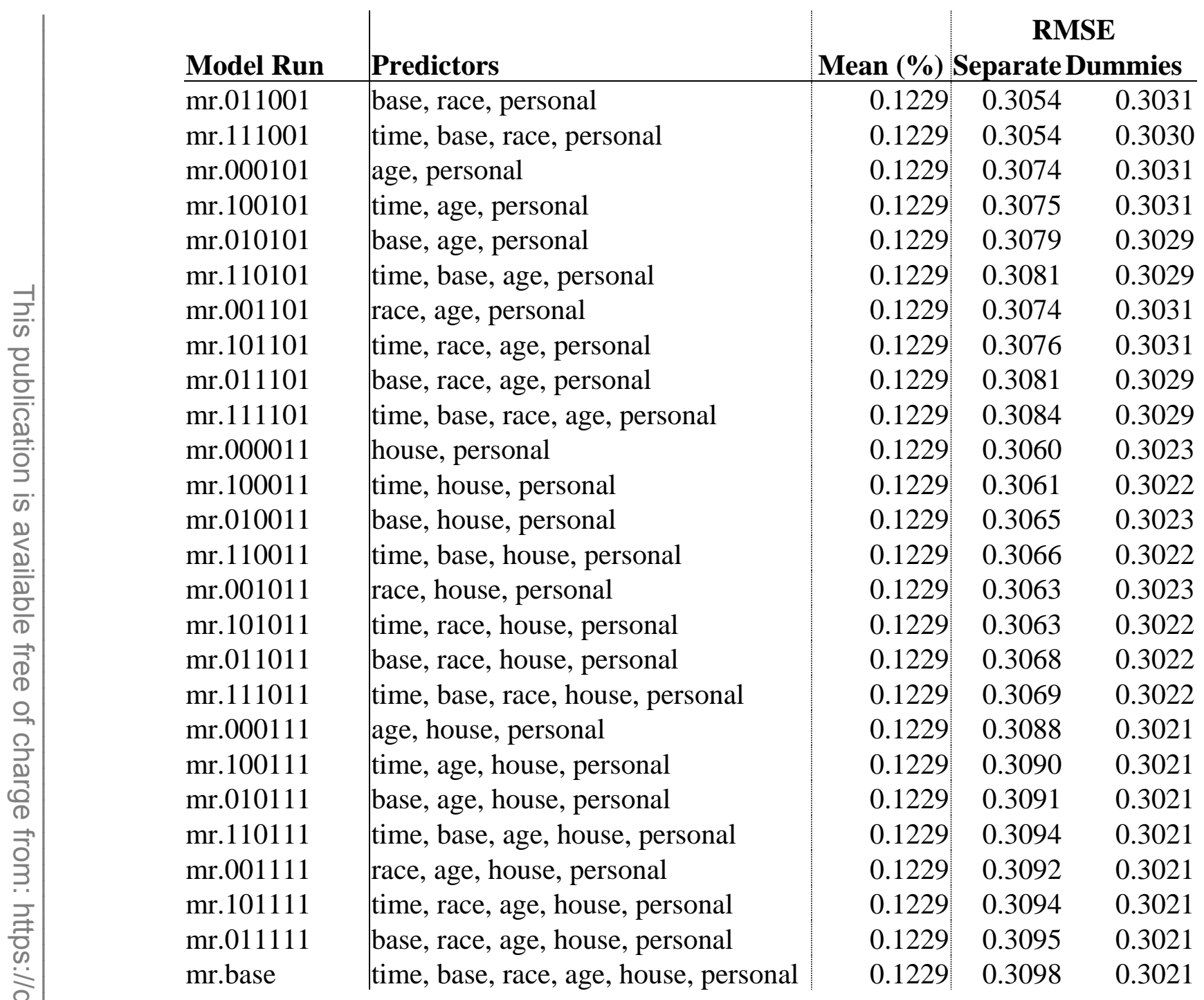


Table 19: RMS Errors of models of fire injury for medium hazard properties.

Model(s) with the lowest RMS Error are in bold. A blank field indicates that the relevant model was not run.

\begin{tabular}{|c|c|c|c|c|}
\hline Model Run & Predictors & Mean & \multicolumn{2}{|c|}{$\begin{array}{c}\text { RMSE } \\
\text { Separate Dummies }\end{array}$} \\
\hline constant & & 0.0628 & \multicolumn{2}{|c|}{0.3786} \\
\hline fx & & 0.0628 & \multicolumn{2}{|c|}{0.3777} \\
\hline tract & & 0.0628 & \multicolumn{2}{|c|}{0.5490} \\
\hline lasso.min & time, base, race, age, house, personal & 0.0628 & \multicolumn{2}{|c|}{0.3830} \\
\hline lasso.1se & time, base, race, age, house, personal & 0.0628 & \multicolumn{2}{|c|}{0.3791} \\
\hline rf.0500 & time, base, race, age, house, personal & 0.0628 & \multicolumn{2}{|c|}{0.3816} \\
\hline rf. 2500 & time, base, race, age, house, personal & 0.0628 & \multicolumn{2}{|c|}{0.3812} \\
\hline mr. 100000 & time & 0.0628 & 0.3780 & 0.3776 \\
\hline mr.010000 & base & 0.0628 & 0.3814 & 0.3784 \\
\hline mr. 110000 & time, base & 0.0628 & 0.3816 & 0.3784 \\
\hline mr.001000 & race & 0.0628 & 0.3874 & 0.3925 \\
\hline mr.101000 & time, race & 0.0628 & 0.3875 & 0.3925 \\
\hline mr.011000 & base, race & 0.0628 & 0.3962 & 0.4781 \\
\hline mr.111000 & time, base, race & 0.0628 & 0.3953 & 0.4781 \\
\hline mr.000100 & age & 0.0628 & 0.3773 & 0.3779 \\
\hline mr. 100100 & time, age & 0.0628 & 0.3775 & 0.3779 \\
\hline mr.010100 & base, age & 0.0628 & 0.3813 & 0.3918 \\
\hline mr. 110100 & time, base, age & 0.0628 & 0.3814 & 0.3928 \\
\hline mr.001100 & race, age & 0.0628 & 0.3769 & 0.3891 \\
\hline mr.101100 & time, race, age & 0.0628 & 0.3768 & 0.3894 \\
\hline mr.011100 & base, race, age & 0.0628 & 0.3814 & 0.4078 \\
\hline mr.111100 & time, base, race, age & 0.0628 & 0.3811 & 0.4085 \\
\hline mr.000010 & house & 0.0628 & 0.5522 & 0.3788 \\
\hline mr. 100010 & time, house & 0.0628 & 0.5541 & 0.3787 \\
\hline mr.010010 & base, house & 0.0628 & 0.5733 & 0.3788 \\
\hline mr.110010 & time, base, house & 0.0628 & 0.5775 & 0.3787 \\
\hline mr.001010 & race, house & 0.0628 & 1.1627 & 0.3799 \\
\hline mr.101010 & time, race, house & 0.0628 & 1.1654 & 0.3797 \\
\hline mr.011010 & base, race, house & 0.0628 & 0.9225 & 0.3815 \\
\hline mr.111010 & time, base, race, house & 0.0628 & 0.9254 & 0.3814 \\
\hline mr.000110 & age, house & 0.0628 & 0.7159 & 0.3795 \\
\hline mr. 100110 & time, age, house & 0.0628 & 0.7155 & 0.3793 \\
\hline mr.010110 & base, age, house & 0.0628 & 0.7082 & 0.3785 \\
\hline mr. 110110 & time, base, age, house & 0.0628 & 0.7082 & 0.3785 \\
\hline mr.001110 & race, age, house & 0.0628 & 0.7995 & 0.3820 \\
\hline mr. 101110 & time, race, age, house & 0.0628 & 0.8011 & 0.3818 \\
\hline mr.011110 & base, race, age, house & 0.0628 & 0.8089 & 0.3830 \\
\hline mr.111110 & time, base, race, age, house & 0.0628 & 0.8087 & 0.3830 \\
\hline mr.000001 & personal & 0.0628 & 0.3784 & 0.3761 \\
\hline mr. 100001 & time, personal & 0.0628 & 0.3790 & 0.3761 \\
\hline mr.010001 & base, personal & 0.0628 & 0.3846 & 0.3778 \\
\hline
\end{tabular}




\begin{tabular}{l|l|l|ll} 
& & \multicolumn{2}{|c}{ RMSE } \\
Model Run & Predictors & Mean & Separate Dummies \\
\hline mr.110001 & time, base, personal & 0.0628 & 0.3847 & 0.3778 \\
mr.001001 & race, personal & 0.0628 & 0.3815 & 0.3788 \\
mr.101001 & time, race, personal & 0.0628 & 0.3823 & 0.3788 \\
mr.011001 & base, race, personal & 0.0628 & 0.3970 & 0.3825 \\
mr.111001 & time, base, race, personal & 0.0628 & 0.3982 & 0.3825 \\
mr.000101 & age, personal & 0.0628 & $\mathbf{0 . 3 7 5 4}$ & 0.3820 \\
mr.100101 & time, age, personal & 0.0628 & 0.3755 & 0.3823 \\
mr.010101 & base, age, personal & 0.0628 & 0.3805 & 0.3775 \\
mr.110101 & time, base, age, personal & 0.0628 & 0.3806 & 0.3775 \\
mr.001101 & race, age, personal & 0.0628 & 0.3757 & 0.3824 \\
mr.101101 & time, race, age, personal & 0.0628 & 0.3758 & 0.3826 \\
mr.011101 & base, race, age, personal & 0.0628 & 0.3857 & 0.3800 \\
mr.111101 & time, base, race, age, personal & 0.0628 & 0.3861 & 0.3800 \\
mr.000011 & house, personal & 0.0628 & 0.9446 & 0.3786 \\
mr.100011 & time, house, personal & 0.0628 & 0.9890 & 0.3786 \\
mr.010011 & base, house, personal & 0.0628 & 0.8528 & 0.3793 \\
mr.110011 & time, base, house, personal & 0.0628 & 0.9016 & 0.3793 \\
mr.001011 & race, house, personal & 0.0628 & 2.6674 & 0.3825 \\
mr.101011 & time, race, house, personal & 0.0628 & 2.8300 & 0.3825 \\
mr.011011 & base, race, house, personal & 0.0628 & 2.1459 & 0.3850 \\
mr.111011 & time, base, race, house, personal & 0.0628 & 2.3017 & 0.3851 \\
mr.000111 & age, house, personal & 0.0628 & 0.9614 & 0.3847 \\
mr.100111 & time, age, house, personal & 0.0628 & 0.9599 & 0.3847 \\
mr.010111 & base, age, house, personal & 0.0628 & 1.1853 & 0.3848 \\
mr.110111 & time, base, age, house, personal & 0.0628 & 1.1909 & 0.3848 \\
mr.001111 & race, age, house, personal & 0.0628 & 1.3700 & 0.3851 \\
mr.101111 & time, race, age, house, personal & 0.0628 & 1.3842 & 0.3851 \\
mr.011111 & base, race, age, house, personal & 0.0628 & 1.5971 & 0.3868 \\
& time, base, race, age, house, personal & 0.0628 & 1.6196 & 0.3868
\end{tabular}


Table 20: RMS Errors of models of fire deaths for medium hazard properties.

Model(s) with the lowest RMS Error are in bold. A blank field indicates that the relevant model was not run.

\begin{tabular}{|c|c|c|c|c|}
\hline Model Run & Predictors & Mean & \multicolumn{2}{|c|}{$\begin{array}{l}\text { RMSE } \\
\text { rate Dummies }\end{array}$} \\
\hline constant & & 0.0044 & \multicolumn{2}{|c|}{0.0831} \\
\hline fx & & 0.0044 & \multicolumn{2}{|c|}{0.0832} \\
\hline tract & & 0.0044 & \multicolumn{2}{|c|}{0.1176} \\
\hline lasso.min & time, base, race, age, house, personal & 0.0044 & \multicolumn{2}{|c|}{0.0831} \\
\hline lasso.1se & time, base, race, age, house, personal & 0.0044 & \multicolumn{2}{|c|}{0.0831} \\
\hline rf.0500 & time, base, race, age, house, personal & 0.0044 & \multicolumn{2}{|c|}{0.0855} \\
\hline rf. 2500 & time, base, race, age, house, personal & 0.0044 & \multicolumn{2}{|c|}{0.0852} \\
\hline mr. 100000 & time & 0.0044 & 0.0832 & 0.0832 \\
\hline mr.010000 & base & 0.0044 & 0.0832 & 0.0832 \\
\hline mr.110000 & time, base & 0.0044 & 0.0833 & 0.0832 \\
\hline mr.001000 & race & 0.0044 & 0.0837 & 0.0832 \\
\hline mr.101000 & time, race & 0.0044 & 0.0838 & 0.0832 \\
\hline mr.011000 & base, race & 0.0044 & 0.0842 & 0.0838 \\
\hline mr.111000 & time, base, race & 0.0044 & 0.0844 & 0.0838 \\
\hline mr.000100 & age & 0.0044 & 0.0873 & 0.0832 \\
\hline mr.100100 & time, age & 0.0044 & 0.0971 & 0.0832 \\
\hline mr.010100 & base, age & 0.0044 & 0.0846 & 0.0835 \\
\hline mr.110100 & time, base, age & 0.0044 & 0.0845 & 0.0835 \\
\hline mr.001100 & race, age & 0.0044 & 0.1149 & 0.0833 \\
\hline mr.101100 & time, race, age & 0.0044 & 0.1092 & 0.0833 \\
\hline mr.011100 & base, race, age & 0.0044 & 0.0862 & 0.0838 \\
\hline mr.111100 & time, base, race, age & 0.0044 & 0.0860 & 0.0838 \\
\hline mr.000010 & house & 0.0044 & 0.0834 & 0.0832 \\
\hline mr.100010 & time, house & 0.0044 & 0.0835 & 0.0832 \\
\hline mr.010010 & base, house & 0.0044 & 0.0834 & 0.0832 \\
\hline mr.110010 & time, base, house & 0.0044 & 0.0836 & 0.0832 \\
\hline $\mathrm{mr} .001010$ & race, house & 0.0044 & 0.0836 & 0.0832 \\
\hline mr.101010 & time, race, house & 0.0044 & 0.0843 & 0.0832 \\
\hline mr.011010 & base, race, house & 0.0044 & 0.0838 & 0.0832 \\
\hline mr.111010 & time, base, race, house & 0.0044 & 0.0842 & 0.0832 \\
\hline mr.000110 & age, house & 0.0044 & 0.0898 & 0.0832 \\
\hline mr.100110 & time, age, house & 0.0044 & 0.1267 & 0.0832 \\
\hline mr.010110 & base, age, house & 0.0044 & 0.0876 & 0.0832 \\
\hline mr.110110 & time, base, age, house & 0.0044 & 0.0938 & 0.0832 \\
\hline mr.001110 & race, age, house & 0.0044 & 0.0857 & 0.0832 \\
\hline mr.101110 & time, race, age, house & 0.0044 & 0.0857 & 0.0832 \\
\hline mr.011110 & base, race, age, house & 0.0044 & 0.4781 & 0.0832 \\
\hline mr.111110 & time, base, race, age, house & 0.0044 & 0.1750 & 0.0832 \\
\hline mr.000001 & personal & 0.0044 & 0.0833 & 0.0832 \\
\hline mr.100001 & time, personal & 0.0044 & 0.0833 & 0.0832 \\
\hline mr.010001 & base, personal & 0.0044 & 0.0833 & 0.0833 \\
\hline
\end{tabular}




\begin{tabular}{|c|c|c|c|c|c|}
\hline & Model Run & Predictors & Mean & $\begin{array}{r}\text { RM } \\
\text { Separate } 1\end{array}$ & mmies \\
\hline & mr.110001 & time, base, personal & 0.0044 & 0.0833 & 0.0833 \\
\hline & mr.001001 & race, personal & 0.0044 & 0.0835 & 0.0832 \\
\hline & mr.101001 & time, race, personal & 0.0044 & 0.0837 & 0.0832 \\
\hline & mr.011001 & base, race, personal & 0.0044 & 0.0835 & 0.0834 \\
\hline & mr.111001 & time, base, race, personal & 0.0044 & 0.0838 & 0.0834 \\
\hline & mr.000101 & age, personal & 0.0044 & 0.0930 & 0.0833 \\
\hline & mr.100101 & time, age, personal & 0.0044 & 0.1030 & 0.0833 \\
\hline & mr.010101 & base, age, personal & 0.0044 & 0.0855 & 0.0833 \\
\hline & mr.110101 & time, base, age, personal & 0.0044 & 0.0867 & 0.0833 \\
\hline & mr.001101 & race, age, personal & 0.0044 & 0.1126 & 0.0833 \\
\hline & mr.101101 & time, race, age, personal & 0.0044 & 0.1883 & 0.0833 \\
\hline & mr.011101 & base, race, age, personal & 0.0044 & 0.0911 & 0.0833 \\
\hline & mr.111101 & time, base, race, age, personal & 0.0044 & 0.1078 & 0.0833 \\
\hline & mr.000011 & house, personal & 0.0044 & 0.0837 & 0.0832 \\
\hline & mr.100011 & time, house, personal & 0.0044 & 0.0840 & 0.0832 \\
\hline & mr.010011 & base, house, personal & 0.0044 & 0.0837 & 0.0832 \\
\hline & mr.110011 & time, base, house, personal & 0.0044 & 0.0845 & 0.0832 \\
\hline & mr.001011 & race, house, personal & 0.0044 & 0.0838 & 0.0832 \\
\hline & mr.101011 & time, race, house, personal & 0.0044 & 0.0844 & 0.0832 \\
\hline & mr.011011 & base, race, house, personal & 0.0044 & 0.0944 & 0.0832 \\
\hline & mr.111011 & time, base, race, house, personal & 0.0044 & 307.3813 & 0.0832 \\
\hline & mr.000111 & age, house, personal & 0.0044 & 0.4783 & 0.0832 \\
\hline & mr.100111 & time, age, house, personal & 0.0044 & $1.85 \mathrm{E}+25$ & 0.0832 \\
\hline & mr.010111 & base, age, house, personal & 0.0044 & 0.0940 & 0.0833 \\
\hline & mr.110111 & time, base, age, house, personal & 0.0044 & 0.1398 & 0.0833 \\
\hline & mr.001111 & race, age, house, personal & 0.0044 & 0.0959 & 0.0832 \\
\hline & mr.101111 & time, race, age, house, personal & 0.0044 & 0.7341 & 0.0832 \\
\hline & mr.011111 & base, race, age, house, personal & 0.0044 & 0.0932 & 0.0832 \\
\hline & mr.base & time, base, race, age, house, personal & 0.0044 & 0.1434 & 0.0833 \\
\hline
\end{tabular}




\section{Appendix D: High-Hazard Properties: Model Results}

Table 21: RMS Errors of models of fire risk for high hazard properties.

Model(s) with the lowest RMS Error are in bold. A blank field indicates that the relevant model was not run.

\begin{tabular}{|c|c|c|c|c|}
\hline \multirow[b]{2}{*}{ Model Run } & \multirow[b]{2}{*}{ Predictors } & \multirow[b]{2}{*}{ Mean } & \multicolumn{2}{|c|}{ RMSE } \\
\hline & & & Separate & Dummies \\
\hline constant & & 0.0996 & \multicolumn{2}{|c|}{0.6967} \\
\hline rsk.clss & risk class & 0.0996 & \multicolumn{2}{|c|}{0.6964} \\
\hline lasso.min & risk class, time, high.rise, base, race, age, house, personal & 0.0996 & \multicolumn{2}{|c|}{0.8986} \\
\hline lasso.1se & risk class, time, high.rise, base, race, age, house, personal & 0.0996 & \multirow{2}{*}{\multicolumn{2}{|c|}{$\begin{array}{l}0.9370 \\
\mathbf{0 . 6 1 9 0}\end{array}$}} \\
\hline rForest & risk class, time, high.rise, base, race, age, house, personal & 0.0996 & & \\
\hline hr.0000000 & risk class & 0.0996 & 0.6904 & 0.6920 \\
\hline hr.0000001 & risk class, personal & 0.0996 & 0.6841 & 0.6913 \\
\hline hr.0000010 & risk class, house & 0.0996 & 0.6893 & 0.6840 \\
\hline hr.0000011 & risk class, house, personal & 0.0996 & 0.6821 & 0.6708 \\
\hline hr.0000100 & risk class, age & 0.0996 & 0.6883 & 0.7519 \\
\hline hr.0000101 & risk class, age, personal & 0.0996 & 0.6822 & 0.7236 \\
\hline hr.0000110 & risk class, age, house & 0.0996 & 0.6858 & 0.6961 \\
\hline hr.0000111 & risk class, age, house, personal & 0.0996 & 0.6794 & 0.6785 \\
\hline hr.0001000 & risk class, race & 0.0996 & 0.6892 & 0.7801 \\
\hline hr.0001001 & risk class, race, personal & 0.0996 & 0.6868 & 0.6862 \\
\hline hr.0001010 & risk class, race, house & 0.0996 & 0.6879 & 0.7317 \\
\hline hr.0001011 & risk class, race, house, personal & 0.0996 & 0.6855 & 0.7193 \\
\hline hr.0001100 & risk class, race, age & 0.0996 & 0.6862 & 0.6909 \\
\hline hr.0001101 & risk class, race, age, personal & 0.0996 & 0.6830 & 0.6753 \\
\hline hr.0001110 & risk class, race, age, house & 0.0996 & 0.6843 & 0.7129 \\
\hline hr.0001111 & risk class, race, age, house, personal & 0.0996 & 0.6821 & 0.6850 \\
\hline hr.0010000 & risk class, base & 0.0996 & 0.6883 & 0.7014 \\
\hline hr.0010001 & risk class, base, personal & 0.0996 & 0.6834 & 0.6774 \\
\hline hr.0010010 & risk class, base, house & 0.0996 & 0.6885 & 0.6908 \\
\hline hr.0010011 & risk class, base, house, personal & 0.0996 & 0.6824 & 0.6684 \\
\hline hr.0010100 & risk class, base, age & 0.0996 & 0.6880 & 0.7098 \\
\hline hr.0010101 & risk class, base, age, personal & 0.0996 & 0.6816 & 0.6802 \\
\hline hr.0010110 & risk class, base, age, house & 0.0996 & 0.6887 & 0.6825 \\
\hline hr.0010111 & risk class, base, age, house, personal & 0.0996 & 0.6788 & 0.6684 \\
\hline hr.0011000 & risk class, base, race & 0.0996 & 0.6883 & 0.6999 \\
\hline hr.0011001 & risk class, base, race, personal & 0.0996 & 0.6869 & 0.6779 \\
\hline hr.0011010 & risk class, base, race, house & 0.0996 & 0.6918 & 0.9260 \\
\hline hr.0011011 & risk class, base, race, house, personal & 0.0996 & 0.6861 & 0.7410 \\
\hline hr.0011100 & risk class, base, race, age & 0.0996 & 0.6869 & 0.7126 \\
\hline hr.0011101 & risk class, base, race, age, personal & 0.0996 & 0.6849 & 0.6799 \\
\hline hr.0011110 & risk class, base, race, age, house & 0.0996 & 0.6863 & 0.8090 \\
\hline hr.0011111 & risk class, base, race, age, house, personal & 0.0996 & 0.6825 & 0.7380 \\
\hline hr.0100000 & risk class, high.rise & 0.0996 & 0.6901 & 0.6906 \\
\hline hr.0100001 & risk class, high.rise, personal & 0.0996 & 0.6825 & 0.6870 \\
\hline hr.0100010 & risk class, high.rise, house & 0.0996 & 0.6891 & 0.6826 \\
\hline hr.0100011 & risk class, high.rise, house, personal & 0.0996 & 0.6798 & 0.6655 \\
\hline hr.0100100 & risk class, high.rise, age & 0.0996 & 0.6880 & 0.7655 \\
\hline hr.0100101 & risk class, high.rise, age, personal & 0.0996 & 0.6805 & 0.7165 \\
\hline hr.0100110 & risk class, high.rise, age, house & 0.0996 & 0.6858 & 0.6963 \\
\hline hr.0100111 & risk class, high.rise, age, house, personal & 0.0996 & 0.6771 & 0.6804 \\
\hline hr.0101000 & risk class, high.rise, race & 0.0996 & 0.6883 & 0.7633 \\
\hline hr.0101001 & risk class, high.rise, race, personal & 0.0996 & 0.6846 & 0.6769 \\
\hline hr.0101010 & risk class, high.rise, race, house & 0.0996 & 0.6858 & 0.7057 \\
\hline hr.0101011 & risk class, high.rise, race, house, personal & 0.0996 & 0.6824 & 0.6881 \\
\hline hr.0101100 & risk class, high.rise, race, age & 0.0996 & 0.6854 & 0.6880 \\
\hline hr.0101101 & risk class, high.rise, race, age, personal & 0.0996 & 0.6811 & 0.6686 \\
\hline
\end{tabular}




\begin{tabular}{|c|c|c|c|c|}
\hline \multirow[b]{2}{*}{ Model Run } & \multirow[b]{2}{*}{ Predictors } & \multirow[b]{2}{*}{ Mean } & \multicolumn{2}{|c|}{ RMSE } \\
\hline & & & Separate & Dummies \\
\hline hr.0101110 & risk class, high.rise, race, age, house & 0.0996 & 0.6830 & 0.6887 \\
\hline hr.0101111 & risk class, high.rise, race, age, house, personal & 0.0996 & 0.6796 & 0.6707 \\
\hline hr.0110000 & risk class, high.rise, base & 0.0996 & 0.6880 & 0.7057 \\
\hline hr.0110001 & risk class, high.rise, base, personal & 0.0996 & 0.6819 & 0.6730 \\
\hline hr.0110010 & risk class, high.rise, base, house & 0.0996 & 0.6885 & 0.6907 \\
\hline hr.0110011 & risk class, high.rise, base, house, personal & 0.0996 & 0.6798 & 0.6627 \\
\hline hr.0110100 & risk class, high.rise, base, age & 0.0996 & 0.6875 & 0.7249 \\
\hline hr.0110101 & risk class, high.rise, base, age, personal & 0.0996 & 0.6799 & 0.6762 \\
\hline hr.0110110 & risk class, high.rise, base, age, house & 0.0996 & 0.6894 & 0.6847 \\
\hline hr.0110111 & risk class, high.rise, base, age, house, personal & 0.0996 & 0.6768 & 0.6698 \\
\hline hr.0111000 & risk class, high.rise, base, race & 0.0996 & 0.6870 & 0.6904 \\
\hline hr.0111001 & risk class, high.rise, base, race, personal & 0.0996 & 0.6848 & 0.6676 \\
\hline hr.0111010 & risk class, high.rise, base, race, house & 0.0996 & 0.6897 & 0.8122 \\
\hline hr.0111011 & risk class, high.rise, base, race, house, personal & 0.0996 & 0.6829 & 0.6943 \\
\hline hr.0111100 & risk class, high.rise, base, race, age & 0.0996 & 0.6861 & 0.7029 \\
\hline hr.0111101 & risk class, high.rise, base, race, age, personal & 0.0996 & 0.6832 & 0.6697 \\
\hline hr.0111110 & risk class, high.rise, base, race, age, house & 0.0996 & 0.6854 & 0.7556 \\
\hline hr.0111111 & risk class, high.rise, base, race, age, house, personal & 0.0996 & 0.6802 & 0.6960 \\
\hline hr. 1000000 & risk class, time & 0.0996 & 0.6905 & 0.6911 \\
\hline hr.1000001 & risk class, time, personal & 0.0996 & 0.6842 & 0.6842 \\
\hline hr. 1000010 & risk class, time, house & 0.0996 & 0.6895 & 0.6821 \\
\hline hr.1000011 & risk class, time, house, personal & 0.0996 & 0.6820 & 0.6659 \\
\hline hr.1000100 & risk class, time, age & 0.0996 & 0.6886 & 0.7120 \\
\hline hr.1000101 & risk class, time, age, personal & 0.0996 & 0.6823 & 0.6948 \\
\hline hr.1000110 & risk class, time, age, house & 0.0996 & 0.6860 & 0.6823 \\
\hline hr.1000111 & risk class, time, age, house, personal & 0.0996 & 0.6796 & 0.6686 \\
\hline hr.1001000 & risk class, time, race & 0.0996 & 0.6890 & 0.7722 \\
\hline hr.1001001 & risk class, time, race, personal & 0.0996 & 0.6873 & 0.6828 \\
\hline hr.1001010 & risk class, time, race, house & 0.0996 & 0.6874 & 0.7322 \\
\hline hr.1001011 & risk class, time, race, house, personal & 0.0996 & 0.6851 & 0.7170 \\
\hline hr.1001100 & risk class, time, race, age & 0.0996 & 0.6873 & 0.6870 \\
\hline hr.1001101 & risk class, time, race, age, personal & 0.0996 & 0.6838 & 0.6707 \\
\hline hr.1001110 & risk class, time, race, age, house & 0.0996 & 0.6850 & 0.7130 \\
\hline hr.1001111 & risk class, time, race, age, house, personal & 0.0996 & 0.6823 & 0.6828 \\
\hline hr.1010000 & risk class, time, base & 0.0996 & 0.6884 & 0.7049 \\
\hline hr.1010001 & risk class, time, base, personal & 0.0996 & 0.6836 & 0.6748 \\
\hline hr.1010010 & risk class, time, base, house & 0.0996 & 0.6893 & 0.6895 \\
\hline hr.1010011 & risk class, time, base, house, personal & 0.0996 & 0.6823 & 0.6666 \\
\hline hr.1010100 & risk class, time, base, age & 0.0996 & 0.6882 & 0.6998 \\
\hline hr.1010101 & risk class, time, base, age, personal & 0.0996 & 0.6819 & 0.6736 \\
\hline hr.1010110 & risk class, time, base, age, house & 0.0996 & 0.6903 & 0.6821 \\
\hline hr.1010111 & risk class, time, base, age, house, personal & 0.0996 & 0.6794 & 0.6670 \\
\hline hr.1011000 & risk class, time, base, race & 0.0996 & 0.6889 & 0.6962 \\
\hline hr.1011001 & risk class, time, base, race, personal & 0.0996 & 0.6873 & 0.6758 \\
\hline hr.1011010 & risk class, time, base, race, house & 0.0996 & 0.6915 & 0.9203 \\
\hline hr.1011011 & risk class, time, base, race, house, personal & 0.0996 & 0.6857 & 0.7379 \\
\hline hr. 1011100 & risk class, time, base, race, age & 0.0996 & 0.6884 & 0.7089 \\
\hline hr.1011101 & risk class, time, base, race, age, personal & 0.0996 & 0.6863 & 0.6773 \\
\hline hr.1011110 & risk class, time, base, race, age, house & 0.0996 & 0.6880 & 0.8086 \\
\hline hr.1011111 & risk class, time, base, race, age, house, personal & 0.0996 & 0.6829 & 0.7343 \\
\hline hr. 1100000 & risk class, time, high.rise & 0.0996 & 0.6902 & 0.6897 \\
\hline hr.1100001 & risk class, time, high.rise, personal & 0.0996 & 0.6827 & 0.6796 \\
\hline hr.1100010 & risk class, time, high.rise, house & 0.0996 & 0.6893 & 0.6805 \\
\hline hr.1100011 & risk class, time, high.rise, house, personal & 0.0996 & 0.6798 & 0.6602 \\
\hline hr. 1100100 & risk class, time, high.rise, age & 0.0996 & 0.6883 & 0.7267 \\
\hline hr.1100101 & risk class, time, high.rise, age, personal & 0.0996 & 0.6806 & 0.6893 \\
\hline hr.1100110 & risk class, time, high.rise, age, house & 0.0996 & 0.6860 & 0.6825 \\
\hline
\end{tabular}




\begin{tabular}{|c|c|c|c|c|}
\hline \multirow[b]{2}{*}{ Model Run } & \multirow[b]{2}{*}{ Predictors } & \multirow[b]{2}{*}{ Mean } & \multicolumn{2}{|c|}{ RMSE } \\
\hline & & & Separate & Dummies \\
\hline hr.1100111 & risk class, time, high.rise, age, house, personal & 0.0996 & 0.6773 & 0.6695 \\
\hline hr.1101000 & risk class, time, high.rise, race & 0.0996 & 0.6881 & 0.7551 \\
\hline hr.1101001 & risk class, time, high.rise, race, personal & 0.0996 & 0.6850 & 0.6735 \\
\hline hr.1101010 & risk class, time, high.rise, race, house & 0.0996 & 0.6854 & 0.7042 \\
\hline hr.1101011 & risk class, time, high.rise, race, house, personal & 0.0996 & 0.6821 & 0.6860 \\
\hline hr. 1101100 & risk class, time, high.rise, race, age & 0.0996 & 0.6864 & 0.6866 \\
\hline hr.1101101 & risk class, time, high.rise, race, age, personal & 0.0996 & 0.6819 & 0.6642 \\
\hline hr.1101110 & risk class, time, high.rise, race, age, house & 0.0996 & 0.6837 & 0.6881 \\
\hline hr.1101111 & risk class, time, high.rise, race, age, house, personal & 0.0996 & 0.6799 & 0.6685 \\
\hline hr.1110000 & risk class, time, high.rise, base & 0.0996 & 0.6881 & 0.7091 \\
\hline hr.1110001 & risk class, time, high.rise, base, personal & 0.0996 & 0.6821 & 0.6699 \\
\hline hr.1110010 & risk class, time, high.rise, base, house & 0.0996 & 0.6895 & 0.6909 \\
\hline hr.1110011 & risk class, time, high.rise, base, house, personal & 0.0996 & 0.6799 & 0.6606 \\
\hline hr.1110100 & risk class, time, high.rise, base, age & 0.0996 & 0.6877 & 0.7191 \\
\hline hr.1110101 & risk class, time, high.rise, base, age, personal & 0.0996 & 0.6802 & 0.6707 \\
\hline hr.1110110 & risk class, time, high.rise, base, age, house & 0.0996 & 0.6911 & 0.6862 \\
\hline hr.1110111 & risk class, time, high.rise, base, age, house, personal & 0.0996 & 0.6774 & 0.6684 \\
\hline hr.1111000 & risk class, time, high.rise, base, race & 0.0996 & 0.6876 & 0.6863 \\
\hline hr.1111001 & risk class, time, high.rise, base, race, personal & 0.0996 & 0.6852 & 0.6654 \\
\hline hr.1111010 & risk class, time, high.rise, base, race, house & 0.0996 & 0.6898 & 0.8079 \\
\hline hr.1111011 & risk class, time, high.rise, base, race, house, personal & 0.0996 & 0.6826 & 0.6911 \\
\hline hr.1111100 & risk class, time, high.rise, base, race, age & 0.0996 & 0.6875 & 0.7002 \\
\hline hr.1111101 & risk class, time, high.rise, base, race, age, personal & 0.0996 & 0.6845 & 0.6672 \\
\hline hr.1111110 & risk class, time, high.rise, base, race, age, house & 0.0996 & 0.6871 & 0.7549 \\
\hline hr.1111111 & risk class, time, high.rise, base, race, age, house, personal & 0.0996 & 0.6808 & 0.6937 \\
\hline
\end{tabular}


Table 22: RMS Errors of models of percent of fires that grow beyond the room of origin for high hazard properties.

Model(s) with the lowest RMS Error are in bold. A blank field indicates that the relevant model was not run.

\begin{tabular}{|c|c|c|c|c|}
\hline \multirow[b]{2}{*}{ Model Run } & \multirow[b]{2}{*}{ Predictors } & \multirow{2}{*}{$\begin{array}{c}\text { Mean } \\
(\%)\end{array}$} & \multicolumn{2}{|c|}{ RMSE } \\
\hline & & & Dummies & Separate \\
\hline constant & & 0.0568 & \multicolumn{2}{|c|}{0.2148} \\
\hline rsk.clss & risk class & 0.0568 & \multicolumn{2}{|c|}{0.2105} \\
\hline lasso.min & risk class, time, high.rise, base, race, age, house, personal & 0.0568 & \multicolumn{2}{|c|}{0.9259} \\
\hline lasso.1se & risk class, time, high.rise, base, race, age, house, personal & 0.0568 & \multirow{2}{*}{\multicolumn{2}{|c|}{$\begin{array}{l}0.9267 \\
\mathbf{0 . 1 5 9 7}\end{array}$}} \\
\hline rForest & risk class, time, high.rise, base, race, age, house, personal & 0.0568 & & \\
\hline hr.0000000 & risk class & 0.0568 & 0.2091 & 0.2043 \\
\hline hr.0000001 & risk class, personal & 0.0568 & 0.2081 & 0.1956 \\
\hline hr.0000010 & risk class, house & 0.0568 & 0.2068 & 0.1965 \\
\hline hr.0000011 & risk class, house, personal & 0.0568 & 0.2054 & 0.1894 \\
\hline hr.0000100 & risk class, age & 0.0568 & 0.2078 & 0.1945 \\
\hline hr.0000101 & risk class, age, personal & 0.0568 & 0.2070 & 0.1874 \\
\hline hr.0000110 & risk class, age, house & 0.0568 & 0.2057 & 0.1908 \\
\hline hr.0000111 & risk class, age, house, personal & 0.0568 & 0.2041 & 0.1845 \\
\hline hr.0001000 & risk class, race & 0.0568 & 0.2091 & 0.2033 \\
\hline hr.0001001 & risk class, race, personal & 0.0568 & 0.2079 & 0.1938 \\
\hline hr.0001010 & risk class, race, house & 0.0568 & 0.2067 & 0.1951 \\
\hline hr.0001011 & risk class, race, house, personal & 0.0568 & 0.2050 & 0.1876 \\
\hline hr.0001100 & risk class, race, age & 0.0568 & 0.2080 & 0.1937 \\
\hline hr.0001101 & risk class, race, age, personal & 0.0568 & 0.2068 & 0.1868 \\
\hline hr.0001110 & risk class, race, age, house & 0.0568 & 0.2058 & 0.1899 \\
\hline hr.0001111 & risk class, race, age, house, personal & 0.0568 & 0.2040 & 0.1844 \\
\hline hr.0010000 & risk class, base & 0.0568 & 0.2088 & 0.2004 \\
\hline hr.0010001 & risk class, base, personal & 0.0568 & 0.2075 & 0.1942 \\
\hline hr.0010010 & risk class, base, house & 0.0568 & 0.2055 & 0.1917 \\
\hline hr.0010011 & risk class, base, house, personal & 0.0568 & 0.2047 & 0.1871 \\
\hline hr.0010100 & risk class, base, age & 0.0568 & 0.2064 & 0.1923 \\
\hline hr.0010101 & risk class, base, age, personal & 0.0568 & 0.2055 & 0.1871 \\
\hline hr.0010110 & risk class, base, age, house & 0.0568 & 0.2048 & 0.1892 \\
\hline hr.0010111 & risk class, base, age, house, personal & 0.0568 & 0.2034 & 0.1845 \\
\hline hr.0011000 & risk class, base, race & 0.0568 & 0.2087 & 0.1998 \\
\hline hr.0011001 & risk class, base, race, personal & 0.0568 & 0.2074 & 0.1926 \\
\hline hr.0011010 & risk class, base, race, house & 0.0568 & 0.2056 & 0.1911 \\
\hline hr.0011011 & risk class, base, race, house, personal & 0.0568 & 0.2047 & 0.1862 \\
\hline hr.0011100 & risk class, base, race, age & 0.0568 & 0.2066 & 0.1912 \\
\hline hr.0011101 & risk class, base, race, age, personal & 0.0568 & 0.2056 & 0.1865 \\
\hline hr.0011110 & risk class, base, race, age, house & 0.0568 & 0.2048 & 0.1883 \\
\hline hr.0011111 & risk class, base, race, age, house, personal & 0.0568 & 0.2035 & 0.1844 \\
\hline hr.0100000 & risk class, high.rise & 0.0568 & 0.2081 & 0.2039 \\
\hline hr.0100001 & risk class, high.rise, personal & 0.0568 & 0.2075 & 0.1949 \\
\hline hr.0100010 & risk class, high.rise, house & 0.0568 & 0.2069 & 0.1964 \\
\hline hr.0100011 & risk class, high.rise, house, personal & 0.0568 & 0.2056 & 0.1896 \\
\hline hr.0100100 & risk class, high.rise, age & 0.0568 & 0.2072 & 0.1931 \\
\hline hr.0100101 & risk class, high.rise, age, personal & 0.0568 & 0.2065 & 0.1869 \\
\hline hr.0100110 & risk class, high.rise, age, house & 0.0568 & 0.2060 & 0.1901 \\
\hline hr.0100111 & risk class, high.rise, age, house, personal & 0.0568 & 0.2044 & 0.1842 \\
\hline hr.0101000 & risk class, high.rise, race & 0.0568 & 0.2081 & 0.2030 \\
\hline hr.0101001 & risk class, high.rise, race, personal & 0.0568 & 0.2073 & 0.1936 \\
\hline hr.0101010 & risk class, high.rise, race, house & 0.0568 & 0.2068 & 0.1945 \\
\hline hr.0101011 & risk class, high.rise, race, house, personal & 0.0568 & 0.2053 & 0.1880 \\
\hline hr.0101100 & risk class, high.rise, race, age & 0.0568 & 0.2074 & 0.1922 \\
\hline hr.0101101 & risk class, high.rise, race, age, personal & 0.0568 & 0.2065 & 0.1862 \\
\hline
\end{tabular}




\begin{tabular}{|c|c|c|c|c|}
\hline \multirow[b]{2}{*}{ Model Run } & \multirow[b]{2}{*}{ Predictors } & \multirow{2}{*}{$\begin{array}{c}\text { Mean } \\
(\%)\end{array}$} & \multicolumn{2}{|c|}{ RMSE } \\
\hline & & & Dummies & Separate \\
\hline hr.0101110 & risk class, high.rise, race, age, house & 0.0568 & 0.2061 & 0.1891 \\
\hline hr.0101111 & risk class, high.rise, race, age, house, personal & 0.0568 & 0.2045 & 0.1841 \\
\hline hr.0110000 & risk class, high.rise, base & 0.0568 & 0.2081 & 0.1997 \\
\hline hr.0110001 & risk class, high.rise, base, personal & 0.0568 & 0.2070 & 0.1930 \\
\hline hr.0110010 & risk class, high.rise, base, house & 0.0568 & 0.2057 & 0.1916 \\
\hline hr.0110011 & risk class, high.rise, base, house, personal & 0.0568 & 0.2050 & 0.1866 \\
\hline hr.0110100 & risk class, high.rise, base, age & 0.0568 & 0.2061 & 0.1910 \\
\hline hr.0110101 & risk class, high.rise, base, age, personal & 0.0568 & 0.2053 & 0.1866 \\
\hline hr.0110110 & risk class, high.rise, base, age, house & 0.0568 & 0.2051 & 0.1883 \\
\hline hr.0110111 & risk class, high.rise, base, age, house, personal & 0.0568 & 0.2038 & 0.1841 \\
\hline hr.0111000 & risk class, high.rise, base, race & 0.0568 & 0.2080 & 0.1988 \\
\hline hr.0111001 & risk class, high.rise, base, race, personal & 0.0568 & 0.2070 & 0.1912 \\
\hline hr.0111010 & risk class, high.rise, base, race, house & 0.0568 & 0.2058 & 0.1904 \\
\hline hr.0111011 & risk class, high.rise, base, race, house, personal & 0.0568 & 0.2050 & 0.1855 \\
\hline hr.0111100 & risk class, high.rise, base, race, age & 0.0568 & 0.2063 & 0.1899 \\
\hline hr.0111101 & risk class, high.rise, base, race, age, personal & 0.0568 & 0.2054 & 0.1859 \\
\hline hr.0111110 & risk class, high.rise, base, race, age, house & 0.0568 & 0.2051 & 0.1871 \\
\hline hr.0111111 & risk class, high.rise, base, race, age, house, personal & 0.0568 & 0.2039 & 0.1840 \\
\hline hr. 1000000 & risk class, time & 0.0568 & 0.2087 & 0.2021 \\
\hline hr.1000001 & risk class, time, personal & 0.0568 & 0.2079 & 0.1922 \\
\hline hr.1000010 & risk class, time, house & 0.0568 & 0.2063 & 0.1950 \\
\hline hr.1000011 & risk class, time, house, personal & 0.0568 & 0.2049 & 0.1880 \\
\hline hr. 1000100 & risk class, time, age & 0.0568 & 0.2075 & 0.1938 \\
\hline hr.1000101 & risk class, time, age, personal & 0.0568 & 0.2067 & 0.1869 \\
\hline hr.1000110 & risk class, time, age, house & 0.0568 & 0.2053 & 0.1894 \\
\hline hr.1000111 & risk class, time, age, house, personal & 0.0568 & 0.2035 & 0.1837 \\
\hline hr.1001000 & risk class, time, race & 0.0568 & 0.2087 & 0.2013 \\
\hline hr.1001001 & risk class, time, race, personal & 0.0568 & 0.2076 & 0.1903 \\
\hline hr.1001010 & risk class, time, race, house & 0.0568 & 0.2062 & 0.1940 \\
\hline hr.1001011 & risk class, time, race, house, personal & 0.0568 & 0.2046 & 0.1864 \\
\hline hr.1001100 & risk class, time, race, age & 0.0568 & 0.2078 & 0.1932 \\
\hline hr.1001101 & risk class, time, race, age, personal & 0.0568 & 0.2066 & 0.1863 \\
\hline hr.1001110 & risk class, time, race, age, house & 0.0568 & 0.2054 & 0.1884 \\
\hline hr.1001111 & risk class, time, race, age, house, personal & 0.0568 & 0.2035 & 0.1837 \\
\hline hr. 1010000 & risk class, time, base & 0.0568 & 0.2083 & 0.1981 \\
\hline hr.1010001 & risk class, time, base, personal & 0.0568 & 0.2071 & 0.1908 \\
\hline hr.1010010 & risk class, time, base, house & 0.0568 & 0.2051 & 0.1904 \\
\hline hr.1010011 & risk class, time, base, house, personal & 0.0568 & 0.2043 & 0.1860 \\
\hline hr.1010100 & risk class, time, base, age & 0.0568 & 0.2061 & 0.1914 \\
\hline hr.1010101 & risk class, time, base, age, personal & 0.0568 & 0.2051 & 0.1867 \\
\hline hr.1010110 & risk class, time, base, age, house & 0.0568 & 0.2044 & 0.1879 \\
\hline hr.1010111 & risk class, time, base, age, house, personal & 0.0568 & 0.2029 & 0.1836 \\
\hline hr.1011000 & risk class, time, base, race & 0.0568 & 0.2082 & 0.1976 \\
\hline hr.1011001 & risk class, time, base, race, personal & 0.0568 & 0.2070 & 0.1891 \\
\hline hr.1011010 & risk class, time, base, race, house & 0.0568 & 0.2053 & 0.1898 \\
\hline hr.1011011 & risk class, time, base, race, house, personal & 0.0568 & 0.2043 & 0.1847 \\
\hline hr.1011100 & risk class, time, base, race, age & 0.0568 & 0.2063 & 0.1905 \\
\hline hr.1011101 & risk class, time, base, race, age, personal & 0.0568 & 0.2052 & 0.1861 \\
\hline hr.1011110 & risk class, time, base, race, age, house & 0.0568 & 0.2045 & 0.1871 \\
\hline hr.1011111 & risk class, time, base, race, age, house, personal & 0.0568 & 0.2029 & 0.1837 \\
\hline hr.1100000 & risk class, time, high.rise & 0.0568 & 0.2076 & 0.2019 \\
\hline hr.1100001 & risk class, time, high.rise, personal & 0.0568 & 0.2071 & 0.1918 \\
\hline hr.1100010 & risk class, time, high.rise, house & 0.0568 & 0.2064 & 0.1944 \\
\hline hr.1100011 & risk class, time, high.rise, house, personal & 0.0568 & 0.2052 & 0.1883 \\
\hline hr.1100100 & risk class, time, high.rise, age & 0.0568 & 0.2069 & 0.1922 \\
\hline hr.1100101 & risk class, time, high.rise, age, personal & 0.0568 & 0.2063 & 0.1866 \\
\hline hr.1100110 & risk class, time, high.rise, age, house & 0.0568 & 0.2055 & 0.1888 \\
\hline
\end{tabular}




\begin{tabular}{|c|c|c|c|c|}
\hline \multirow[b]{2}{*}{ Model Run } & \multirow[b]{2}{*}{ Predictors } & \multirow{2}{*}{$\begin{array}{c}\text { Mean } \\
(\%)\end{array}$} & \multicolumn{2}{|c|}{ RMSE } \\
\hline & & & Dummies & Separate \\
\hline hr.1100111 & risk class, time, high.rise, age, house, personal & 0.0568 & 0.2039 & 0.1835 \\
\hline hr.1101000 & risk class, time, high.rise, race & 0.0568 & 0.2076 & 0.2010 \\
\hline hr.1101001 & risk class, time, high.rise, race, personal & 0.0568 & 0.2069 & 0.1907 \\
\hline hr.1101010 & risk class, time, high.rise, race, house & 0.0568 & 0.2063 & 0.1931 \\
\hline hr.1101011 & risk class, time, high.rise, race, house, personal & 0.0568 & 0.2048 & 0.1867 \\
\hline hr.1101100 & risk class, time, high.rise, race, age & 0.0568 & 0.2072 & 0.1914 \\
\hline hr.1101101 & risk class, time, high.rise, race, age, personal & 0.0568 & 0.2062 & 0.1860 \\
\hline hr.1101110 & risk class, time, high.rise, race, age, house & 0.0568 & 0.2056 & 0.1880 \\
\hline hr.1101111 & risk class, time, high.rise, race, age, house, personal & 0.0568 & 0.2040 & 0.1836 \\
\hline hr.1110000 & risk class, time, high.rise, base & 0.0568 & 0.2075 & 0.1968 \\
\hline hr.1110001 & risk class, time, high.rise, base, personal & 0.0568 & 0.2066 & 0.1904 \\
\hline hr.1110010 & risk class, time, high.rise, base, house & 0.0568 & 0.2053 & 0.1899 \\
\hline hr.1110011 & risk class, time, high.rise, base, house, personal & 0.0568 & 0.2046 & 0.1857 \\
\hline hr.1110100 & risk class, time, high.rise, base, age & 0.0568 & 0.2058 & 0.1903 \\
\hline hr.1110101 & risk class, time, high.rise, base, age, personal & 0.0568 & 0.2049 & 0.1862 \\
\hline hr.1110110 & risk class, time, high.rise, base, age, house & 0.0568 & 0.2047 & 0.1872 \\
\hline hr.1110111 & risk class, time, high.rise, base, age, house, personal & 0.0568 & 0.2033 & 0.1833 \\
\hline hr.1111000 & risk class, time, high.rise, base, race & 0.0568 & 0.2075 & 0.1962 \\
\hline hr.1111001 & risk class, time, high.rise, base, race, personal & 0.0568 & 0.2066 & 0.1885 \\
\hline hr.1111010 & risk class, time, high.rise, base, race, house & 0.0568 & 0.2055 & 0.1889 \\
\hline hr.1111011 & risk class, time, high.rise, base, race, house, personal & 0.0568 & 0.2046 & 0.1843 \\
\hline hr.1111100 & risk class, time, high.rise, base, race, age & 0.0568 & 0.2060 & 0.1894 \\
\hline hr.1111101 & risk class, time, high.rise, base, race, age, personal & 0.0568 & 0.2051 & 0.1857 \\
\hline hr.1111110 & risk class, time, high.rise, base, race, age, house & 0.0568 & 0.2047 & 0.1864 \\
\hline hr.1111111 & risk class, time, high.rise, base, race, age, house, personal & 0.0568 & 0.2033 & 0.1833 \\
\hline
\end{tabular}


Table 23: RMS Errors of models of percent of fires that grow beyond the structure of origin for high hazard properties.

Model(s) with the lowest RMS Error are in bold. A blank field indicates that the relevant model was not run.

\begin{tabular}{|c|c|c|c|c|}
\hline \multirow[b]{2}{*}{ Model Run } & \multirow[b]{2}{*}{ Predictors } & \multirow{2}{*}{$\begin{array}{c}\text { Mean } \\
(\%)\end{array}$} & \multicolumn{2}{|c|}{ RMSE } \\
\hline & & & Dummies & Separate \\
\hline constant & & 0.0892 & \multicolumn{2}{|c|}{0.2774} \\
\hline rsk.clss & risk class & 0.0892 & \multicolumn{2}{|c|}{0.2689} \\
\hline lasso.min & risk class, time, high.rise, base, race, age, house, personal & 0.0892 & \multicolumn{2}{|c|}{0.8957} \\
\hline lasso.1se & risk class, time, high.rise, base, race, age, house, personal & 0.0892 & \multirow{2}{*}{\multicolumn{2}{|c|}{$\begin{array}{l}0.8782 \\
\mathbf{0 1 9 4 8}\end{array}$}} \\
\hline rForest & risk class, time, high.rise, base, race, age, house, personal & 0.0892 & & \\
\hline hr.0000000 & risk class & 0.0892 & 0.2684 & 0.2702 \\
\hline hr.0000001 & risk class, personal & 0.0892 & 0.2654 & 0.2391 \\
\hline hr.0000010 & risk class, house & 0.0892 & 0.2617 & 0.2425 \\
\hline hr.0000011 & risk class, house, personal & 0.0892 & 0.2583 & 0.2543 \\
\hline hr.0000100 & risk class, age & 0.0892 & 0.2580 & 0.2334 \\
\hline hr.0000101 & risk class, age, personal & 0.0892 & 0.2536 & 0.2484 \\
\hline hr.0000110 & risk class, age, house & 0.0892 & 0.2509 & 0.2558 \\
\hline hr.0000111 & risk class, age, house, personal & 0.0892 & 0.2475 & 0.2552 \\
\hline hr.0001000 & risk class, race & 0.0892 & 0.2639 & 0.2336 \\
\hline hr.0001001 & risk class, race, personal & 0.0892 & 0.2607 & 0.2302 \\
\hline hr.0001010 & risk class, race, house & 0.0892 & 0.2583 & 0.2466 \\
\hline hr.0001011 & risk class, race, house, personal & 0.0892 & 0.2553 & 0.2484 \\
\hline hr.0001100 & risk class, race, age & 0.0892 & 0.2548 & 0.2463 \\
\hline hr.0001101 & risk class, race, age, personal & 0.0892 & 0.2512 & 0.2560 \\
\hline hr.0001110 & risk class, race, age, house & 0.0892 & 0.2478 & 0.2685 \\
\hline hr.0001111 & risk class, race, age, house, personal & 0.0892 & 0.2454 & 0.2845 \\
\hline hr.0010000 & risk class, base & 0.0892 & 0.2685 & 0.2633 \\
\hline hr.0010001 & risk class, base, personal & 0.0892 & 0.2654 & 0.2348 \\
\hline hr.0010010 & risk class, base, house & 0.0892 & 0.2574 & 0.2477 \\
\hline hr.0010011 & risk class, base, house, personal & 0.0892 & 0.2506 & 0.2471 \\
\hline hr.0010100 & risk class, base, age & 0.0892 & 0.2571 & 0.2414 \\
\hline hr.0010101 & risk class, base, age, personal & 0.0892 & 0.2523 & 0.2496 \\
\hline hr.0010110 & risk class, base, age, house & 0.0892 & 0.2451 & 0.2635 \\
\hline hr.0010111 & risk class, base, age, house, personal & 0.0892 & 0.2403 & 0.2652 \\
\hline hr.0011000 & risk class, base, race & 0.0892 & 0.2620 & 0.2209 \\
\hline hr.0011001 & risk class, base, race, personal & 0.0892 & 0.2590 & 0.2344 \\
\hline hr.0011010 & risk class, base, race, house & 0.0892 & 0.2519 & 0.2463 \\
\hline hr.0011011 & risk class, base, race, house, personal & 0.0892 & 0.2478 & 0.2463 \\
\hline hr.0011100 & risk class, base, race, age & 0.0892 & 0.2537 & 0.2541 \\
\hline hr.0011101 & risk class, base, race, age, personal & 0.0892 & 0.2498 & 0.2581 \\
\hline hr.0011110 & risk class, base, race, age, house & 0.0892 & 0.2403 & 0.2680 \\
\hline hr.0011111 & risk class, base, race, age, house, personal & 0.0892 & 0.2382 & 0.2849 \\
\hline hr.0100000 & risk class, high.rise & 0.0892 & 0.2679 & 0.2759 \\
\hline hr.0100001 & risk class, high.rise, personal & 0.0892 & 0.2651 & 0.2383 \\
\hline hr.0100010 & risk class, high.rise, house & 0.0892 & 0.2615 & 0.2449 \\
\hline hr.0100011 & risk class, high.rise, house, personal & 0.0892 & 0.2580 & 0.2607 \\
\hline hr.0100100 & risk class, high.rise, age & 0.0892 & 0.2577 & 0.2404 \\
\hline hr.0100101 & risk class, high.rise, age, personal & 0.0892 & 0.2535 & 0.2550 \\
\hline hr.0100110 & risk class, high.rise, age, house & 0.0892 & 0.2506 & 0.2701 \\
\hline hr.0100111 & risk class, high.rise, age, house, personal & 0.0892 & 0.2474 & 0.2642 \\
\hline hr.0101000 & risk class, high.rise, race & 0.0892 & 0.2640 & 0.2403 \\
\hline hr.0101001 & risk class, high.rise, race, personal & 0.0892 & 0.2610 & 0.2323 \\
\hline hr.0101010 & risk class, high.rise, race, house & 0.0892 & 0.2584 & 0.2502 \\
\hline hr.0101011 & risk class, high.rise, race, house, personal & 0.0892 & 0.2553 & 0.2511 \\
\hline hr.0101100 & risk class, high.rise, race, age & 0.0892 & 0.2549 & 0.2504 \\
\hline hr.0101101 & risk class, high.rise, race, age, personal & 0.0892 & 0.2514 & 0.2629 \\
\hline
\end{tabular}




\begin{tabular}{|c|c|c|c|c|}
\hline \multirow[b]{2}{*}{ Model Run } & \multirow[b]{2}{*}{ Predictors } & \multirow{2}{*}{$\begin{array}{c}\text { Mean } \\
(\%)\end{array}$} & \multicolumn{2}{|c|}{ RMSE } \\
\hline & & & Dummies & Separate \\
\hline hr.0101110 & risk class, high.rise, race, age, house & 0.0892 & 0.2476 & 0.2732 \\
\hline hr.0101111 & risk class, high.rise, race, age, house, personal & 0.0892 & 0.2455 & 0.2822 \\
\hline hr.0110000 & risk class, high.rise, base & 0.0892 & 0.2682 & 0.2683 \\
\hline hr.0110001 & risk class, high.rise, base, personal & 0.0892 & 0.2649 & 0.2389 \\
\hline hr.0110010 & risk class, high.rise, base, house & 0.0892 & 0.2573 & 0.2532 \\
\hline hr.0110011 & risk class, high.rise, base, house, personal & 0.0892 & 0.2506 & 0.2582 \\
\hline hr.0110100 & risk class, high.rise, base, age & 0.0892 & 0.2567 & 0.2471 \\
\hline hr.0110101 & risk class, high.rise, base, age, personal & 0.0892 & 0.2518 & 0.2643 \\
\hline hr.0110110 & risk class, high.rise, base, age, house & 0.0892 & 0.2450 & 0.2689 \\
\hline hr.0110111 & risk class, high.rise, base, age, house, personal & 0.0892 & 0.2404 & 0.2631 \\
\hline hr.0111000 & risk class, high.rise, base, race & 0.0892 & 0.2619 & 0.2279 \\
\hline hr.0111001 & risk class, high.rise, base, race, personal & 0.0892 & 0.2589 & 0.2415 \\
\hline hr.0111010 & risk class, high.rise, base, race, house & 0.0892 & 0.2517 & 0.2571 \\
\hline hr.0111011 & risk class, high.rise, base, race, house, personal & 0.0892 & 0.2478 & 0.2586 \\
\hline hr.0111100 & risk class, high.rise, base, race, age & 0.0892 & 0.2535 & 0.2513 \\
\hline hr.0111101 & risk class, high.rise, base, race, age, personal & 0.0892 & 0.2495 & 0.2669 \\
\hline hr.0111110 & risk class, high.rise, base, race, age, house & 0.0892 & 0.2403 & 0.2696 \\
\hline hr.0111111 & risk class, high.rise, base, race, age, house, personal & 0.0892 & 0.2384 & 0.2956 \\
\hline hr.1000000 & risk class, time & 0.0892 & 0.2504 & 0.2155 \\
\hline hr.1000001 & risk class, time, personal & 0.0892 & 0.2442 & 0.2191 \\
\hline hr.1000010 & risk class, time, house & 0.0892 & 0.2453 & 0.2283 \\
\hline hr.1000011 & risk class, time, house, personal & 0.0892 & 0.2416 & 0.2469 \\
\hline hr. 1000100 & risk class, time, age & 0.0892 & 0.2451 & 0.2384 \\
\hline hr.1000101 & risk class, time, age, personal & 0.0892 & 0.2389 & 0.2541 \\
\hline hr.1000110 & risk class, time, age, house & 0.0892 & 0.2407 & 0.2620 \\
\hline hr.1000111 & risk class, time, age, house, personal & 0.0892 & 0.2360 & 0.2702 \\
\hline hr.1001000 & risk class, time, race & 0.0892 & 0.2457 & 0.2154 \\
\hline hr.1001001 & risk class, time, race, personal & 0.0892 & 0.2419 & 0.2285 \\
\hline hr.1001010 & risk class, time, race, house & 0.0892 & 0.2435 & 0.2369 \\
\hline hr.1001011 & risk class, time, race, house, personal & 0.0892 & 0.2403 & 0.2498 \\
\hline hr. 1001100 & risk class, time, race, age & 0.0892 & 0.2424 & 0.2575 \\
\hline hr.1001101 & risk class, time, race, age, personal & 0.0892 & 0.2371 & 0.2700 \\
\hline hr.1001110 & risk class, time, race, age, house & 0.0892 & 0.2385 & 0.2674 \\
\hline hr.1001111 & risk class, time, race, age, house, personal & 0.0892 & 0.2347 & 0.2873 \\
\hline hr. 1010000 & risk class, time, base & 0.0892 & 0.2496 & 0.2155 \\
\hline hr.1010001 & risk class, time, base, personal & 0.0892 & 0.2439 & 0.2267 \\
\hline hr.1010010 & risk class, time, base, house & 0.0892 & 0.2436 & 0.2291 \\
\hline hr.1010011 & risk class, time, base, house, personal & 0.0892 & 0.2383 & 0.2397 \\
\hline hr. 1010100 & risk class, time, base, age & 0.0892 & 0.2448 & 0.2436 \\
\hline hr.1010101 & risk class, time, base, age, personal & 0.0892 & 0.2384 & 0.2572 \\
\hline hr.1010110 & risk class, time, base, age, house & 0.0892 & 0.2381 & 0.2587 \\
\hline hr.1010111 & risk class, time, base, age, house, personal & 0.0892 & 0.2328 & 0.2852 \\
\hline hr.1011000 & risk class, time, base, race & 0.0892 & 0.2444 & 0.2161 \\
\hline hr.1011001 & risk class, time, base, race, personal & 0.0892 & 0.2404 & 0.2277 \\
\hline hr.1011010 & risk class, time, base, race, house & 0.0892 & 0.2409 & 0.2439 \\
\hline hr.1011011 & risk class, time, base, race, house, personal & 0.0892 & 0.2369 & 0.2505 \\
\hline hr.1011100 & risk class, time, base, race, age & 0.0892 & 0.2418 & 0.2479 \\
\hline hr.1011101 & risk class, time, base, race, age, personal & 0.0892 & 0.2365 & 0.2646 \\
\hline hr.1011110 & risk class, time, base, race, age, house & 0.0892 & 0.2348 & 0.2718 \\
\hline hr.1011111 & risk class, time, base, race, age, house, personal & 0.0892 & 0.2314 & 0.2896 \\
\hline hr.1100000 & risk class, time, high.rise & 0.0892 & 0.2493 & 0.2221 \\
\hline hr.1100001 & risk class, time, high.rise, personal & 0.0892 & 0.2439 & 0.2253 \\
\hline hr.1100010 & risk class, time, high.rise, house & 0.0892 & 0.2441 & 0.2358 \\
\hline hr.1100011 & risk class, time, high.rise, house, personal & 0.0892 & 0.2410 & 0.2534 \\
\hline hr.1100100 & risk class, time, high.rise, age & 0.0892 & 0.2443 & 0.2446 \\
\hline hr.1100101 & risk class, time, high.rise, age, personal & 0.0892 & 0.2387 & 0.2639 \\
\hline hr. 1100110 & risk class, time, high.rise, age, house & 0.0892 & 0.2396 & 0.2650 \\
\hline
\end{tabular}




\begin{tabular}{|c|c|c|c|c|}
\hline \multirow[b]{2}{*}{ Model Run } & \multirow[b]{2}{*}{ Predictors } & \multirow{2}{*}{$\begin{array}{c}\text { Mean } \\
(\%)\end{array}$} & \multicolumn{2}{|c|}{ RMSE } \\
\hline & & & Dummies & Separate \\
\hline hr.1100111 & risk class, time, high.rise, age, house, personal & 0.0892 & 0.2356 & 0.2749 \\
\hline hr. 1101000 & risk class, time, high.rise, race & 0.0892 & 0.2453 & 0.2225 \\
\hline hr.1101001 & risk class, time, high.rise, race, personal & 0.0892 & 0.2422 & 0.2355 \\
\hline hr. 1101010 & risk class, time, high.rise, race, house & 0.0892 & 0.2429 & 0.2428 \\
\hline hr.1101011 & risk class, time, high.rise, race, house, personal & 0.0892 & 0.2400 & 0.2574 \\
\hline hr.1101100 & risk class, time, high.rise, race, age & 0.0892 & 0.2421 & 0.2577 \\
\hline hr.1101101 & risk class, time, high.rise, race, age, personal & 0.0892 & 0.2372 & 0.2746 \\
\hline hr.1101110 & risk class, time, high.rise, race, age, house & 0.0892 & 0.2377 & 0.2716 \\
\hline hr.1101111 & risk class, time, high.rise, race, age, house, personal & 0.0892 & 0.2346 & 0.2815 \\
\hline hr.1110000 & risk class, time, high.rise, base & 0.0892 & 0.2486 & 0.2222 \\
\hline hr.1110001 & risk class, time, high.rise, base, personal & 0.0892 & 0.2434 & 0.2353 \\
\hline hr. 1110010 & risk class, time, high.rise, base, house & 0.0892 & 0.2424 & 0.2445 \\
\hline hr.1110011 & risk class, time, high.rise, base, house, personal & 0.0892 & 0.2379 & 0.2518 \\
\hline hr.1110100 & risk class, time, high.rise, base, age & 0.0892 & 0.2440 & 0.2517 \\
\hline hr.1110101 & risk class, time, high.rise, base, age, personal & 0.0892 & 0.2379 & 0.2704 \\
\hline hr.1110110 & risk class, time, high.rise, base, age, house & 0.0892 & 0.2373 & 0.2643 \\
\hline hr.1110111 & risk class, time, high.rise, base, age, house, personal & 0.0892 & 0.2326 & 0.2716 \\
\hline hr. 1111000 & risk class, time, high.rise, base, race & 0.0892 & 0.2438 & 0.2230 \\
\hline hr.1111001 & risk class, time, high.rise, base, race, personal & 0.0892 & 0.2405 & 0.2400 \\
\hline hr. 1111010 & risk class, time, high.rise, base, race, house & 0.0892 & 0.2399 & 0.2456 \\
\hline hr.1111011 & risk class, time, high.rise, base, race, house, personal & 0.0892 & 0.2368 & 0.2601 \\
\hline hr. 1111100 & risk class, time, high.rise, base, race, age & 0.0892 & 0.2414 & 0.2548 \\
\hline hr.1111101 & risk class, time, high.rise, base, race, age, personal & 0.0892 & 0.2363 & 0.2801 \\
\hline hr.1111110 & risk class, time, high.rise, base, race, age, house & 0.0892 & 0.2344 & 0.2718 \\
\hline hr.1111111 & risk class, time, high.rise, base, race, age, house, personal & 0.0892 & 0.2315 & 0.2875 \\
\hline
\end{tabular}


Table 24: RMS Errors of models of fire injuries for high hazard properties.

Model(s) with the lowest RMS Error are in bold. A blank field indicates that the relevant model was not run.

\begin{tabular}{|c|c|c|c|c|}
\hline \multirow[b]{2}{*}{ Model Run } & \multirow[b]{2}{*}{ Predictors } & \multirow[b]{2}{*}{ Mean } & \multicolumn{2}{|c|}{ RMSE } \\
\hline & & & Dummies & Separate \\
\hline constant & & 0.0030 & \multicolumn{2}{|c|}{0.1356} \\
\hline rsk.clss & risk class & 0.0030 & \multicolumn{2}{|c|}{0.1356} \\
\hline lasso.min & risk class, time, high.rise, base, race, age, house, personal & 0.0030 & \multicolumn{2}{|c|}{0.6045} \\
\hline lasso.1se & risk class, time, high.rise, base, race, age, house, personal & 0.0030 & \multicolumn{2}{|c|}{0.6071} \\
\hline rForest & risk class, time, high.rise, base, race, age, house, personal & 0.0030 & \multicolumn{2}{|c|}{0.1374} \\
\hline hr.0000000 & risk class & 0.0030 & 0.1355 & 0.1356 \\
\hline hr.0000001 & risk class, personal & 0.0030 & 0.1354 & 0.1487 \\
\hline hr.0000010 & risk class, house & 0.0030 & 0.1355 & 0.1352 \\
\hline hr.0000011 & risk class, house, personal & 0.0030 & 0.1353 & 0.1383 \\
\hline hr.0000100 & risk class, age & 0.0030 & 0.1355 & 0.2024 \\
\hline hr.0000101 & risk class, age, personal & 0.0030 & 0.1355 & 0.1369 \\
\hline hr.0000110 & risk class, age, house & 0.0030 & 0.1354 & $1.68 \mathrm{E}+13$ \\
\hline hr.0000111 & risk class, age, house, personal & 0.0030 & 0.1354 & $1.84 \mathrm{E}+04$ \\
\hline hr.0001000 & risk class, race & 0.0030 & 0.1356 & 0.1378 \\
\hline hr.0001001 & risk class, race, personal & 0.0030 & 0.1354 & 0.1393 \\
\hline hr.0001010 & risk class, race, house & 0.0030 & 0.1354 & 0.1358 \\
\hline hr.0001011 & risk class, race, house, personal & 0.0030 & 0.1354 & 0.1347 \\
\hline hr.0001100 & risk class, race, age & 0.0030 & 0.1355 & 0.1367 \\
\hline hr.0001101 & risk class, race, age, personal & 0.0030 & 0.1355 & $9.38 \mathrm{E}+03$ \\
\hline hr.0001110 & risk class, race, age, house & 0.0030 & 0.1354 & $6.12 E+29$ \\
\hline hr.0001111 & risk class, race, age, house, personal & 0.0030 & 0.1355 & $3.69 \mathrm{E}+04$ \\
\hline hr.0010000 & risk class, base & 0.0030 & 0.1355 & 0.1357 \\
\hline hr.0010001 & risk class, base, personal & 0.0030 & 0.1354 & 0.1366 \\
\hline hr.0010010 & risk class, base, house & 0.0030 & 0.1354 & 0.1351 \\
\hline hr.0010011 & risk class, base, house, personal & 0.0030 & 0.1353 & 35.9960 \\
\hline hr.0010100 & risk class, base, age & 0.0030 & 0.1355 & 0.1407 \\
\hline hr.0010101 & risk class, base, age, personal & 0.0030 & 0.1355 & $2.18 \mathrm{E}+39$ \\
\hline hr.0010110 & risk class, base, age, house & 0.0030 & 0.1354 & $7.64 \mathrm{E}+29$ \\
\hline hr.0010111 & risk class, base, age, house, personal & 0.0030 & 0.1354 & $2.67 \mathrm{E}+04$ \\
\hline hr.0011000 & risk class, base, race & 0.0030 & 0.1355 & 0.1358 \\
\hline hr.0011001 & risk class, base, race, personal & 0.0030 & 0.1354 & 0.1349 \\
\hline hr.0011010 & risk class, base, race, house & 0.0030 & 0.1354 & 0.1386 \\
\hline hr.0011011 & risk class, base, race, house, personal & 0.0030 & 0.1354 & 1.7652 \\
\hline hr.0011100 & risk class, base, race, age & 0.0030 & 0.1355 & 0.1365 \\
\hline hr.0011101 & risk class, base, race, age, personal & 0.0030 & 0.1354 & $1.49 \mathrm{E}+04$ \\
\hline hr.0011110 & risk class, base, race, age, house & 0.0030 & 0.1354 & $1.60 \mathrm{E}+17$ \\
\hline hr.0011111 & risk class, base, race, age, house, personal & 0.0030 & 0.1354 & $5.20 \mathrm{E}+04$ \\
\hline hr.0100000 & risk class, high.rise & 0.0030 & 0.1355 & 0.1356 \\
\hline hr.0100001 & risk class, high.rise, personal & 0.0030 & 0.1354 & 0.1485 \\
\hline hr.0100010 & risk class, high.rise, house & 0.0030 & 0.1354 & 0.1500 \\
\hline hr.0100011 & risk class, high.rise, house, personal & 0.0030 & 0.1352 & 0.1378 \\
\hline hr.0100100 & risk class, high.rise, age & 0.0030 & 0.1354 & 0.2022 \\
\hline hr.0100101 & risk class, high.rise, age, personal & 0.0030 & 0.1354 & 0.1364 \\
\hline hr.0100110 & risk class, high.rise, age, house & 0.0030 & 0.1354 & $1.89 \mathrm{E}+11$ \\
\hline hr.0100111 & risk class, high.rise, age, house, personal & 0.0030 & 0.1353 & $1.87 \mathrm{E}+04$ \\
\hline hr.0101000 & risk class, high.rise, race & 0.0030 & 0.1356 & 0.1376 \\
\hline hr.0101001 & risk class, high.rise, race, personal & 0.0030 & 0.1353 & 0.1359 \\
\hline hr.0101010 & risk class, high.rise, race, house & 0.0030 & 0.1353 & 0.2220 \\
\hline hr.0101011 & risk class, high.rise, race, house, personal & 0.0030 & 0.1353 & 0.1341 \\
\hline hr.0101100 & risk class, high.rise, race, age & 0.0030 & 0.1355 & 0.1363 \\
\hline hr.0101101 & risk class, high.rise, race, age, personal & 0.0030 & 0.1354 & $9.24 \mathrm{E}+03$ \\
\hline hr.0101110 & risk class, high.rise, race, age, house & 0.0030 & 0.1354 & $5.69 \mathrm{E}+34$ \\
\hline hr.0101111 & risk class, high.rise, race, age, house, personal & 0.0030 & 0.1354 & $3.73 \mathrm{E}+04$ \\
\hline
\end{tabular}




\begin{tabular}{|c|c|c|c|c|}
\hline \multirow[b]{2}{*}{ Model Run } & \multirow[b]{2}{*}{ Predictors } & \multirow[b]{2}{*}{ Mean } & \multicolumn{2}{|c|}{ RMSE } \\
\hline & & & Dummies & Separate \\
\hline hr.0110000 & risk class, high.rise, base & 0.0030 & 0.1355 & 0.1357 \\
\hline hr.0110001 & risk class, high.rise, base, personal & 0.0030 & 0.1354 & 0.1364 \\
\hline hr.0110010 & risk class, high.rise, base, house & 0.0030 & 0.1354 & 0.1353 \\
\hline hr.0110011 & risk class, high.rise, base, house, personal & 0.0030 & 0.1352 & 28.4462 \\
\hline hr.0110100 & risk class, high.rise, base, age & 0.0030 & 0.1354 & 0.1402 \\
\hline hr.0110101 & risk class, high.rise, base, age, personal & 0.0030 & 0.1354 & $6.16 \mathrm{E}+38$ \\
\hline hr.0110110 & risk class, high.rise, base, age, house & 0.0030 & 0.1354 & $1.63 \mathrm{E}+30$ \\
\hline hr.0110111 & risk class, high.rise, base, age, house, personal & 0.0030 & 0.1352 & $2.70 \mathrm{E}+04$ \\
\hline hr.0111000 & risk class, high.rise, base, race & 0.0030 & 0.1354 & 0.1356 \\
\hline hr.0111001 & risk class, high.rise, base, race, personal & 0.0030 & 0.1353 & 0.1346 \\
\hline hr.0111010 & risk class, high.rise, base, race, house & 0.0030 & 0.1353 & 0.1369 \\
\hline hr.0111011 & risk class, high.rise, base, race, house, personal & 0.0030 & 0.1352 & 1.2336 \\
\hline hr.0111100 & risk class, high.rise, base, race, age & 0.0030 & 0.1354 & 0.1357 \\
\hline hr.0111101 & risk class, high.rise, base, race, age, personal & 0.0030 & 0.1353 & $1.58 \mathrm{E}+04$ \\
\hline hr.0111110 & risk class, high.rise, base, race, age, house & 0.0030 & 0.1353 & $6.69 \mathrm{E}+06$ \\
\hline hr.0111111 & risk class, high.rise, base, race, age, house, personal & 0.0030 & 0.1353 & $5.27 \mathrm{E}+04$ \\
\hline hr. 1000000 & risk class, time & 0.0030 & 0.1355 & 0.1356 \\
\hline hr.1000001 & risk class, time, personal & 0.0030 & 0.1354 & 0.1391 \\
\hline hr.1000010 & risk class, time, house & 0.0030 & 0.1355 & 0.1350 \\
\hline hr.1000011 & risk class, time, house, personal & 0.0030 & 0.1353 & 0.1359 \\
\hline hr. 1000100 & risk class, time, age & 0.0030 & 0.1355 & 0.2951 \\
\hline hr.1000101 & risk class, time, age, personal & 0.0030 & 0.1355 & 0.1431 \\
\hline hr. 1000110 & risk class, time, age, house & 0.0030 & 0.1354 & $2.20 \mathrm{E}+03$ \\
\hline hr.1000111 & risk class, time, age, house, personal & 0.0030 & 0.1354 & $6.85 \mathrm{E}+05$ \\
\hline hr.1001000 & risk class, time, race & 0.0030 & 0.1356 & 0.1379 \\
\hline hr.1001001 & risk class, time, race, personal & 0.0030 & 0.1354 & 0.1464 \\
\hline hr.1001010 & risk class, time, race, house & 0.0030 & 0.1354 & 0.1356 \\
\hline hr.1001011 & risk class, time, race, house, personal & 0.0030 & 0.1354 & 0.1350 \\
\hline hr. 1001100 & risk class, time, race, age & 0.0030 & 0.1355 & 0.1362 \\
\hline hr.1001101 & risk class, time, race, age, personal & 0.0030 & 0.1354 & $1.03 \mathrm{E}+04$ \\
\hline hr.1001110 & risk class, time, race, age, house & 0.0030 & 0.1354 & $7.13 \mathrm{E}+14$ \\
\hline hr.1001111 & risk class, time, race, age, house, personal & 0.0030 & 0.1355 & $3.22 \mathrm{E}+04$ \\
\hline hr. 1010000 & risk class, time, base & 0.0030 & 0.1355 & 0.1354 \\
\hline hr.1010001 & risk class, time, base, personal & 0.0030 & 0.1354 & 0.1360 \\
\hline hr.1010010 & risk class, time, base, house & 0.0030 & 0.1354 & 0.1351 \\
\hline hr.1010011 & risk class, time, base, house, personal & 0.0030 & 0.1353 & $4.20 \mathrm{E}+10$ \\
\hline hr. 1010100 & risk class, time, base, age & 0.0030 & 0.1355 & 2.1950 \\
\hline hr.1010101 & risk class, time, base, age, personal & 0.0030 & 0.1355 & $5.65 \mathrm{E}+02$ \\
\hline hr.1010110 & risk class, time, base, age, house & 0.0030 & 0.1355 & $7.36 \mathrm{E}+15$ \\
\hline hr.1010111 & risk class, time, base, age, house, personal & 0.0030 & 0.1354 & $2.56 \mathrm{E}+04$ \\
\hline hr. 1011000 & risk class, time, base, race & 0.0030 & 0.1354 & 0.1358 \\
\hline hr.1011001 & risk class, time, base, race, personal & 0.0030 & 0.1354 & 0.1350 \\
\hline hr.1011010 & risk class, time, base, race, house & 0.0030 & 0.1354 & 0.1390 \\
\hline hr.1011011 & risk class, time, base, race, house, personal & 0.0030 & 0.1354 & 0.1371 \\
\hline hr. 1011100 & risk class, time, base, race, age & 0.0030 & 0.1355 & 0.3003 \\
\hline hr.1011101 & risk class, time, base, race, age, personal & 0.0030 & 0.1354 & $1.39 \mathrm{E}+04$ \\
\hline hr. 1011110 & risk class, time, base, race, age, house & 0.0030 & 0.1354 & $4.08 \mathrm{E}+06$ \\
\hline hr.1011111 & risk class, time, base, race, age, house, personal & 0.0030 & 0.1354 & $5.03 \mathrm{E}+04$ \\
\hline hr. 1100000 & risk class, time, high.rise & 0.0030 & 0.1355 & 0.1355 \\
\hline hr.1100001 & risk class, time, high.rise, personal & 0.0030 & 0.1354 & 0.1387 \\
\hline hr.1100010 & risk class, time, high.rise, house & 0.0030 & 0.1354 & 0.1592 \\
\hline hr.1100011 & risk class, time, high.rise, house, personal & 0.0030 & 0.1352 & 0.1351 \\
\hline hr. 1100100 & risk class, time, high.rise, age & 0.0030 & 0.1354 & 0.2198 \\
\hline hr.1100101 & risk class, time, high.rise, age, personal & 0.0030 & 0.1354 & 0.1426 \\
\hline hr.1100110 & risk class, time, high.rise, age, house & 0.0030 & 0.1354 & $2.21 \mathrm{E}+03$ \\
\hline hr.1100111 & risk class, time, high.rise, age, house, personal & 0.0030 & 0.1353 & $1.76 \mathrm{E}+06$ \\
\hline hr.1101000 & risk class, time, high.rise, race & 0.0030 & 0.1356 & 0.1376 \\
\hline
\end{tabular}




\begin{tabular}{|c|c|c|c|c|}
\hline \multirow[b]{2}{*}{ Model Run } & \multirow[b]{2}{*}{ Predictors } & \multirow[b]{2}{*}{ Mean } & \multicolumn{2}{|c|}{ RMSE } \\
\hline & & & Dummies & Separate \\
\hline hr.1101001 & risk class, time, high.rise, race, personal & 0.0030 & 0.1353 & 0.1377 \\
\hline hr.1101010 & risk class, time, high.rise, race, house & 0.0030 & 0.1353 & 0.3407 \\
\hline hr.1101011 & risk class, time, high.rise, race, house, personal & 0.0030 & 0.1353 & 0.1341 \\
\hline hr. 1101100 & risk class, time, high.rise, race, age & 0.0030 & 0.1355 & 0.1354 \\
\hline hr.1101101 & risk class, time, high.rise, race, age, personal & 0.0030 & 0.1354 & $1.08 \mathrm{E}+04$ \\
\hline hr.1101110 & risk class, time, high.rise, race, age, house & 0.0030 & 0.1354 & $1.71 \mathrm{E}+25$ \\
\hline hr.1101111 & risk class, time, high.rise, race, age, house, personal & 0.0030 & 0.1354 & $3.46 \mathrm{E}+04$ \\
\hline hr. 1110000 & risk class, time, high.rise, base & 0.0030 & 0.1355 & 0.1353 \\
\hline hr.1110001 & risk class, time, high.rise, base, personal & 0.0030 & 0.1354 & 0.1355 \\
\hline hr.1110010 & risk class, time, high.rise, base, house & 0.0030 & 0.1354 & 0.1356 \\
\hline hr.1110011 & risk class, time, high.rise, base, house, personal & 0.0030 & 0.1352 & $2.72 \mathrm{E}+10$ \\
\hline hr.1110100 & risk class, time, high.rise, base, age & 0.0030 & 0.1354 & 0.9193 \\
\hline hr.1110101 & risk class, time, high.rise, base, age, personal & 0.0030 & 0.1354 & $3.65 \mathrm{E}+28$ \\
\hline hr.1110110 & risk class, time, high.rise, base, age, house & 0.0030 & 0.1354 & $1.95 \mathrm{E}+18$ \\
\hline hr.1110111 & risk class, time, high.rise, base, age, house, personal & 0.0030 & 0.1352 & $2.61 \mathrm{E}+04$ \\
\hline hr. 1111000 & risk class, time, high.rise, base, race & 0.0030 & 0.1354 & 0.1356 \\
\hline hr.1111001 & risk class, time, high.rise, base, race, personal & 0.0030 & 0.1353 & 0.1348 \\
\hline hr.1111010 & risk class, time, high.rise, base, race, house & 0.0030 & 0.1353 & 0.1400 \\
\hline hr.1111011 & risk class, time, high.rise, base, race, house, personal & 0.0030 & 0.1352 & $3.26 \mathrm{E}+14$ \\
\hline hr.1111100 & risk class, time, high.rise, base, race, age & 0.0030 & 0.1354 & 0.1357 \\
\hline hr.1111101 & risk class, time, high.rise, base, race, age, personal & 0.0030 & 0.1353 & $1.43 \mathrm{E}+04$ \\
\hline hr.1111110 & risk class, time, high.rise, base, race, age, house & 0.0030 & 0.1353 & $2.54 \mathrm{E}+07$ \\
\hline hr.1111111 & risk class, time, high.rise, base, race, age, house, personal & 0.0030 & 0.1353 & $5.11 \mathrm{E}+04$ \\
\hline
\end{tabular}


Table 25: RMS Errors of models of fire deaths for high hazard properties.

Model(s) with the lowest RMS Error are in bold. A blank field indicates that the relevant model was not run.

\begin{tabular}{|c|c|c|c|c|}
\hline \multirow[b]{2}{*}{ Model Run } & \multirow[b]{2}{*}{ Predictors } & \multirow[b]{2}{*}{ Mean } & \multicolumn{2}{|c|}{ RMSE } \\
\hline & & & Dummies & Separate \\
\hline constant & & 0.0002 & \multicolumn{2}{|c|}{0.0157} \\
\hline rsk.clss & risk class & 0.0002 & \multicolumn{2}{|c|}{0.0157} \\
\hline lasso.min & risk class, time, high.rise, base, race, age, house, personal & 0.0002 & \multicolumn{2}{|c|}{0.5901} \\
\hline lasso.1se & risk class, time, high.rise, base, race, age, house, personal & 0.0002 & \multicolumn{2}{|c|}{0.5917} \\
\hline rForest & risk class, time, high.rise, base, race, age, house, personal & 0.0002 & \multicolumn{2}{|c|}{0.0092} \\
\hline hr.0000000 & risk class & 0.0002 & 0.0157 & 0.0157 \\
\hline hr.0000001 & risk class, personal & 0.0002 & 0.0156 & Infinity \\
\hline hr.0000010 & risk class, house & 0.0002 & 0.0156 & 0.0176 \\
\hline hr.0000011 & risk class, house, personal & 0.0002 & 0.0147 & $4.69 \mathrm{E}+09$ \\
\hline hr.0000100 & risk class, age & 0.0002 & 0.0118 & 4.73E+07 \\
\hline hr.0000101 & risk class, age, personal & 0.0002 & 0.0107 & $4.17 \mathrm{E}+20$ \\
\hline hr.0000110 & risk class, age, house & 0.0002 & 0.0107 & $8.46 \mathrm{E}+12$ \\
\hline hr.0000111 & risk class, age, house, personal & 0.0002 & 0.0102 & $2.09 \mathrm{E}+129$ \\
\hline hr.0001000 & risk class, race & 0.0002 & 0.0157 & 0.0160 \\
\hline hr.0001001 & risk class, race, personal & 0.0002 & 0.0155 & $8.31 \mathrm{E}+38$ \\
\hline hr.0001010 & risk class, race, house & 0.0002 & 0.0155 & 0.1351 \\
\hline hr.0001011 & risk class, race, house, personal & 0.0002 & 0.0141 & $1.45 \mathrm{E}+29$ \\
\hline hr.0001100 & risk class, race, age & 0.0002 & 0.0115 & $1.66 \mathrm{E}+28$ \\
\hline hr.0001101 & risk class, race, age, personal & 0.0002 & 0.0105 & $3.29 \mathrm{E}+24$ \\
\hline $\mathrm{hr} .0001110$ & risk class, race, age, house & 0.0002 & 0.0107 & $5.09 \mathrm{E}+20$ \\
\hline hr.0001111 & risk class, race, age, house, personal & 0.0002 & 0.0101 & $7.50 \mathrm{E}+109$ \\
\hline hr.0010000 & risk class, base & 0.0002 & 0.0157 & 0.0157 \\
\hline hr.0010001 & risk class, base, personal & 0.0002 & 0.0158 & $1.53 \mathrm{E}+26$ \\
\hline hr.0010010 & risk class, base, house & 0.0002 & 0.0154 & $1.34 \mathrm{E}+26$ \\
\hline hr.0010011 & risk class, base, house, personal & 0.0002 & 0.0142 & $1.42 \mathrm{E}+00$ \\
\hline hr.0010100 & risk class, base, age & 0.0002 & 0.0111 & $7.19 \mathrm{E}+28$ \\
\hline hr.0010101 & risk class, base, age, personal & 0.0002 & 0.0103 & $9.20 \mathrm{E}+12$ \\
\hline hr.0010110 & risk class, base, age, house & 0.0002 & 0.0099 & $2.11 \mathrm{E}+14$ \\
\hline hr.0010111 & risk class, base, age, house, personal & 0.0002 & 0.0095 & $5.70 \mathrm{E}+79$ \\
\hline hr.0011000 & risk class, base, race & 0.0002 & 0.0157 & 0.0158 \\
\hline hr.0011001 & risk class, base, race, personal & 0.0002 & 0.0153 & $5.25 \mathrm{E}+13$ \\
\hline hr.0011010 & risk class, base, race, house & 0.0002 & 0.0153 & 0.0478 \\
\hline hr.0011011 & risk class, base, race, house, personal & 0.0002 & 0.0139 & Infinity \\
\hline hr.0011100 & risk class, base, race, age & 0.0002 & 0.0109 & $3.49 \mathrm{E}+16$ \\
\hline hr.0011101 & risk class, base, race, age, personal & 0.0002 & 0.0103 & $2.32 \mathrm{E}+55$ \\
\hline hr.0011110 & risk class, base, race, age, house & 0.0002 & 0.0099 & $3.22 \mathrm{E}+10$ \\
\hline hr.0011111 & risk class, base, race, age, house, personal & 0.0002 & 0.0095 & $2.57 \mathrm{E}+49$ \\
\hline hr.0100000 & risk class, high.rise & 0.0002 & 0.0157 & 0.0157 \\
\hline hr.0100001 & risk class, high.rise, personal & 0.0002 & 0.0153 & Infinity \\
\hline hr.0100010 & risk class, high.rise, house & 0.0002 & 0.0156 & 0.0237 \\
\hline hr.0100011 & risk class, high.rise, house, personal & 0.0002 & 0.0146 & Infinity \\
\hline hr.0100100 & risk class, high.rise, age & 0.0002 & 0.0112 & $1.06 \mathrm{E}+84$ \\
\hline hr.0100101 & risk class, high.rise, age, personal & 0.0002 & 0.0104 & Infinity \\
\hline hr.0100110 & risk class, high.rise, age, house & 0.0002 & 0.0106 & $1.75 \mathrm{E}+134$ \\
\hline hr.0100111 & risk class, high.rise, age, house, personal & 0.0002 & 0.0101 & $1.65 E+119$ \\
\hline hr.0101000 & risk class, high.rise, race & 0.0002 & 0.0157 & 0.0162 \\
\hline hr.0101001 & risk class, high.rise, race, personal & 0.0002 & 0.0151 & $1.07 \mathrm{E}+43$ \\
\hline hr.0101010 & risk class, high.rise, race, house & 0.0002 & 0.0155 & 0.0509 \\
\hline hr.0101011 & risk class, high.rise, race, house, personal & 0.0002 & 0.0139 & $2.87 \mathrm{E}+62$ \\
\hline hr.0101100 & risk class, high.rise, race, age & 0.0002 & 0.0109 & $3.31 \mathrm{E}+107$ \\
\hline hr.0101101 & risk class, high.rise, race, age, personal & 0.0002 & 0.0103 & Infinity \\
\hline hr.0101110 & risk class, high.rise, race, age, house & 0.0002 & 0.0106 & Infinity \\
\hline hr.0101111 & risk class, high.rise, race, age, house, personal & 0.0002 & 0.0100 & $2.19 \mathrm{E}+115$ \\
\hline
\end{tabular}




\begin{tabular}{|c|c|c|c|c|}
\hline \multirow[b]{2}{*}{ Model Run } & \multirow[b]{2}{*}{ Predictors } & \multirow[b]{2}{*}{ Mean } & \multicolumn{2}{|c|}{ RMSE } \\
\hline & & & Dummies & Separate \\
\hline hr.0110000 & risk class, high.rise, base & 0.0002 & 0.0157 & 0.0158 \\
\hline hr.0110001 & risk class, high.rise, base, personal & 0.0002 & 0.0152 & 0.0086 \\
\hline hr.0110010 & risk class, high.rise, base, house & 0.0002 & 0.0153 & $7.57 \mathrm{E}+27$ \\
\hline hr.0110011 & risk class, high.rise, base, house, personal & 0.0002 & 0.0139 & $1.31 \mathrm{E}+11$ \\
\hline hr.0110100 & risk class, high.rise, base, age & 0.0002 & 0.0106 & $3.00 \mathrm{E}+92$ \\
\hline hr.0110101 & risk class, high.rise, base, age, personal & 0.0002 & 0.0100 & $4.93 \mathrm{E}+91$ \\
\hline hr.0110110 & risk class, high.rise, base, age, house & 0.0002 & 0.0099 & Infinity \\
\hline hr.0110111 & risk class, high.rise, base, age, house, personal & 0.0002 & 0.0095 & $3.12 \mathrm{E}+99$ \\
\hline hr.0111000 & risk class, high.rise, base, race & 0.0002 & 0.0156 & 0.0161 \\
\hline hr.0111001 & risk class, high.rise, base, race, personal & 0.0002 & 0.0149 & $6.84 \mathrm{E}+21$ \\
\hline hr.0111010 & risk class, high.rise, base, race, house & 0.0002 & 0.0152 & 6.7242 \\
\hline hr.0111011 & risk class, high.rise, base, race, house, personal & 0.0002 & 0.0136 & Infinity \\
\hline hr.0111100 & risk class, high.rise, base, race, age & 0.0002 & 0.0104 & $7.42 \mathrm{E}+122$ \\
\hline hr.0111101 & risk class, high.rise, base, race, age, personal & 0.0002 & 0.0099 & $5.48 \mathrm{E}+89$ \\
\hline hr.0111110 & risk class, high.rise, base, race, age, house & 0.0002 & 0.0099 & Infinity \\
\hline hr.0111111 & risk class, high.rise, base, race, age, house, personal & 0.0002 & 0.0094 & $3.33 \mathrm{E}+104$ \\
\hline hr. 1000000 & risk class, time & 0.0002 & 0.0157 & 0.0157 \\
\hline hr.1000001 & risk class, time, personal & 0.0002 & 0.0155 & $5.43 \mathrm{E}+07$ \\
\hline hr.1000010 & risk class, time, house & 0.0002 & 0.0156 & 0.0268 \\
\hline hr.1000011 & risk class, time, house, personal & 0.0002 & 0.0147 & $3.56 \mathrm{E}+10$ \\
\hline hr.1000100 & risk class, time, age & 0.0002 & 0.0118 & $3.49 \mathrm{E}+09$ \\
\hline hr.1000101 & risk class, time, age, personal & 0.0002 & 0.0107 & $3.34 \mathrm{E}+27$ \\
\hline hr.1000110 & risk class, time, age, house & 0.0002 & 0.0107 & $2.03 \mathrm{E}+18$ \\
\hline hr.1000111 & risk class, time, age, house, personal & 0.0002 & 0.0102 & $5.66 \mathrm{E}+125$ \\
\hline hr.1001000 & risk class, time, race & 0.0002 & 0.0157 & 0.0160 \\
\hline hr.1001001 & risk class, time, race, personal & 0.0002 & 0.0154 & $3.38 \mathrm{E}+16$ \\
\hline hr.1001010 & risk class, time, race, house & 0.0002 & 0.0155 & 1.0271 \\
\hline hr.1001011 & risk class, time, race, house, personal & 0.0002 & 0.0141 & $5.85 \mathrm{E}+06$ \\
\hline hr.1001100 & risk class, time, race, age & 0.0002 & 0.0115 & $1.23 \mathrm{E}+24$ \\
\hline hr.1001101 & risk class, time, race, age, personal & 0.0002 & 0.0105 & Infinity \\
\hline hr.1001110 & risk class, time, race, age, house & 0.0002 & 0.0107 & $8.05 E+30$ \\
\hline hr.1001111 & risk class, time, race, age, house, personal & 0.0002 & 0.0101 & $4.03 E+116$ \\
\hline hr.1010000 & risk class, time, base & 0.0002 & 0.0157 & 0.0304 \\
\hline hr.1010001 & risk class, time, base, personal & 0.0002 & 0.0158 & $1.79 \mathrm{E}+12$ \\
\hline hr.1010010 & risk class, time, base, house & 0.0002 & 0.0154 & $7.40 \mathrm{E}+19$ \\
\hline hr.1010011 & risk class, time, base, house, personal & 0.0002 & 0.0141 & Infinity \\
\hline hr.1010100 & risk class, time, base, age & 0.0002 & 0.0111 & $1.80 \mathrm{E}+15$ \\
\hline hr.1010101 & risk class, time, base, age, personal & 0.0002 & 0.0103 & $8.43 \mathrm{E}+13$ \\
\hline hr.1010110 & risk class, time, base, age, house & 0.0002 & 0.0099 & $5.47 \mathrm{E}+33$ \\
\hline hr.1010111 & risk class, time, base, age, house, personal & 0.0002 & 0.0095 & $1.55 \mathrm{E}+74$ \\
\hline hr.1011000 & risk class, time, base, race & 0.0002 & 0.0157 & $8.43 E+129$ \\
\hline hr.1011001 & risk class, time, base, race, personal & 0.0002 & 0.0153 & 0.0114 \\
\hline hr.1011010 & risk class, time, base, race, house & 0.0002 & 0.0152 & $1.56 \mathrm{E}+07$ \\
\hline hr.1011011 & risk class, time, base, race, house, personal & 0.0002 & 0.0139 & Infinity \\
\hline hr.1011100 & risk class, time, base, race, age & 0.0002 & 0.0109 & $1.05 \mathrm{E}+19$ \\
\hline hr.1011101 & risk class, time, base, race, age, personal & 0.0002 & 0.0103 & $1.97 \mathrm{E}+68$ \\
\hline hr.1011110 & risk class, time, base, race, age, house & 0.0002 & 0.0099 & $1.07 \mathrm{E}+23$ \\
\hline hr.1011111 & risk class, time, base, race, age, house, personal & 0.0002 & 0.0095 & $3.11 \mathrm{E}+73$ \\
\hline hr. 1100000 & risk class, time, high.rise & 0.0002 & 0.0157 & 0.0157 \\
\hline hr.1100001 & risk class, time, high.rise, personal & 0.0002 & 0.0153 & Infinity \\
\hline hr.1100010 & risk class, time, high.rise, house & 0.0002 & 0.0156 & 0.0357 \\
\hline hr.1100011 & risk class, time, high.rise, house, personal & 0.0002 & 0.0146 & Infinity \\
\hline hr.1100100 & risk class, time, high.rise, age & 0.0002 & 0.0112 & $3.67 \mathrm{E}+100$ \\
\hline hr.1100101 & risk class, time, high.rise, age, personal & 0.0002 & 0.0104 & Infinity \\
\hline hr.1100110 & risk class, time, high.rise, age, house & 0.0002 & 0.0106 & $8.22 \mathrm{E}+144$ \\
\hline hr.1100111 & risk class, time, high.rise, age, house, personal & 0.0002 & 0.0101 & $9.72 \mathrm{E}+122$ \\
\hline hr.1101000 & risk class, time, high.rise, race & 0.0002 & 0.0157 & 0.0162 \\
\hline
\end{tabular}




\begin{tabular}{|c|c|c|c|c|}
\hline \multirow[b]{2}{*}{ Model Run } & \multirow[b]{2}{*}{ Predictors } & \multirow[b]{2}{*}{ Mean } & \multicolumn{2}{|c|}{ RMSE } \\
\hline & & & Dummies & Separate \\
\hline hr.1101001 & risk class, time, high.rise, race, personal & 0.0002 & 0.0151 & $1.68 \mathrm{E}+21$ \\
\hline hr.1101010 & risk class, time, high.rise, race, house & 0.0002 & 0.0154 & 0.1798 \\
\hline hr.1101011 & risk class, time, high.rise, race, house, personal & 0.0002 & 0.0138 & $2.86 \mathrm{E}+10$ \\
\hline hr. 1101100 & risk class, time, high.rise, race, age & 0.0002 & 0.0109 & $6.28 \mathrm{E}+96$ \\
\hline hr.1101101 & risk class, time, high.rise, race, age, personal & 0.0002 & 0.0103 & Infinity \\
\hline hr.1101110 & risk class, time, high.rise, race, age, house & 0.0002 & 0.0106 & $2.26 \mathrm{E}+149$ \\
\hline hr.1101111 & risk class, time, high.rise, race, age, house, personal & 0.0002 & 0.0100 & $5.49 \mathrm{E}+106$ \\
\hline hr.1110000 & risk class, time, high.rise, base & 0.0002 & 0.0157 & 0.0301 \\
\hline hr.1110001 & risk class, time, high.rise, base, personal & 0.0002 & 0.0152 & $7.51 \mathrm{E}+12$ \\
\hline hr.1110010 & risk class, time, high.rise, base, house & 0.0002 & 0.0153 & $7.91 \mathrm{E}+18$ \\
\hline hr.1110011 & risk class, time, high.rise, base, house, personal & 0.0002 & 0.0139 & $6.98 \mathrm{E}+03$ \\
\hline hr.1110100 & risk class, time, high.rise, base, age & 0.0002 & 0.0106 & $5.49 \mathrm{E}+71$ \\
\hline hr.1110101 & risk class, time, high.rise, base, age, personal & 0.0002 & 0.0100 & $3.24 \mathrm{E}+22$ \\
\hline hr.1110110 & risk class, time, high.rise, base, age, house & 0.0002 & 0.0098 & Infinity \\
\hline hr.1110111 & risk class, time, high.rise, base, age, house, personal & 0.0002 & 0.0095 & $3.28 \mathrm{E}+96$ \\
\hline hr.1111000 & risk class, time, high.rise, base, race & 0.0002 & 0.0156 & $4.34 \mathrm{E}+130$ \\
\hline hr.1111001 & risk class, time, high.rise, base, race, personal & 0.0002 & 0.0149 & 0.0110 \\
\hline hr.1111010 & risk class, time, high.rise, base, race, house & 0.0002 & 0.0152 & $3.78 \mathrm{E}+06$ \\
\hline hr.1111011 & risk class, time, high.rise, base, race, house, personal & 0.0002 & 0.0136 & Infinity \\
\hline hr.1111100 & risk class, time, high.rise, base, race, age & 0.0002 & 0.0104 & $4.34 \mathrm{E}+96$ \\
\hline hr.1111101 & risk class, time, high.rise, base, race, age, personal & 0.0002 & 0.0099 & $3.83 \mathrm{E}+55$ \\
\hline hr.1111110 & risk class, time, high.rise, base, race, age, house & 0.0002 & 0.0098 & $6.17 \mathrm{E}+146$ \\
\hline hr.1111111 & risk class, time, high.rise, base, race, age, house, personal & 0.0002 & 0.0094 & $6.70 \mathrm{E}+94$ \\
\hline
\end{tabular}




\section{Appendix E: EMS Model Results}

Table 26: RMS Errors of the models for EMS risk based on the 500-cities data set.

Model(s) with the lowest RMS Error are in bold. A blank field indicates that the relevant model was not run.

\begin{tabular}{|c|c|c|c|c|c|c|c|c|c|c|c|c|c|}
\hline Model & 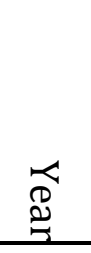 & 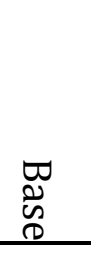 & 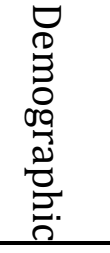 & 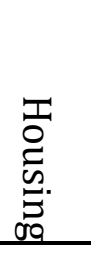 & $\begin{array}{l}\vec{D} \\
0 \\
0 \\
0 \\
0 \\
0 \\
\end{array}$ & $\begin{array}{l}T \\
D \\
\end{array}$ & 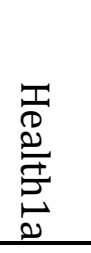 & 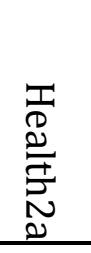 & $\begin{array}{l}\text { Do } \\
\text { og } \\
\text { og. } \\
0 \\
\end{array}$ & 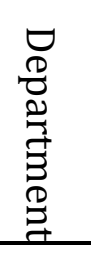 & $\mathrm{S}$ & $\mathrm{L}$ & $\mathrm{C}$ \\
\hline ems.5.const & No & No & No & No & No & No & No & No & No & No & 419.7 & & \\
\hline ems.5.fx & No & No & No & No & No & No & No & No & No & Yes & 389.7 & & \\
\hline ems.5.lasso.1se & Yes & Yes & Yes & Yes & Yes & Yes & Yes & Yes & Yes & No & 407.9 & & \\
\hline ems.5.lasso.min & Yes & Yes & Yes & Yes & Yes & Yes & Yes & Yes & Yes & No & 387.3 & & \\
\hline ems.5.rf & Yes & Yes & Yes & Yes & Yes & Yes & Yes & Yes & Yes & No & 383.1 & & \\
\hline ems.5.000 & Yes & No & No & No & No & No & No & No & No & Yes & 389.6 & $3.90 \mathrm{E}+02$ & 389.6 \\
\hline ems.5.001 & Yes & Yes & No & No & No & No & No & No & No & Yes & 388.5 & $3.87 \mathrm{E}+02$ & 713.4 \\
\hline ems.5.002 & Yes & No & Yes & No & No & No & No & No & Yes & Yes & 509.1 & $2.44 \mathrm{E}+03$ & 464.8 \\
\hline ems.5.003 & Yes & Yes & Yes & No & No & No & No & No & Yes & Yes & 423.6 & $4.87 \mathrm{E}+03$ & 409.9 \\
\hline ems.5.004 & Yes & No & No & Yes & No & No & No & No & No & Yes & 373.3 & $4.48 \mathrm{E}+02$ & 374.4 \\
\hline ems.5.005 & Yes & Yes & No & Yes & No & No & No & No & No & Yes & 373.6 & $3.83 \mathrm{E}+02$ & 373.2 \\
\hline ems.5.006 & Yes & No & Yes & Yes & No & No & No & No & Yes & Yes & 414.0 & $3.67 \mathrm{E}+06$ & 389.1 \\
\hline ems.5.007 & Yes & Yes & Yes & Yes & No & No & No & No & No & Yes & 448.8 & $1.51 \mathrm{E}+06$ & 396.0 \\
\hline ems.5.008 & Yes & No & No & No & Yes & No & No & No & No & Yes & 372.5 & $3.74 \mathrm{E}+02$ & $9.21 \mathrm{E}+06$ \\
\hline ems.5.009 & Yes & Yes & No & No & Yes & No & No & No & Yes & Yes & 372.3 & $3.71 \mathrm{E}+02$ & 371.1 \\
\hline ems.5.010 & Yes & No & Yes & No & Yes & No & No & No & No & Yes & 404.1 & $4.41 \mathrm{E}+04$ & 397.4 \\
\hline ems.5.011 & Yes & Yes & Yes & No & Yes & No & No & No & No & Yes & 378.5 & $3.73 \mathrm{E}+03$ & 372.9 \\
\hline ems.5.012 & Yes & No & No & Yes & Yes & No & No & No & No & Yes & 376.0 & $3.26 \mathrm{E}+15$ & 372.5 \\
\hline ems.5.013 & Yes & Yes & No & Yes & Yes & No & No & No & No & Yes & 372.4 & $4.28 \mathrm{E}+02$ & 371.3 \\
\hline
\end{tabular}




\begin{tabular}{|c|c|c|c|c|c|c|c|c|c|c|c|c|c|}
\hline Model & $\begin{array}{l}\overleftrightarrow{\mathbb{8}} \\
\stackrel{\mathscr{2}}{7}\end{array}$ & 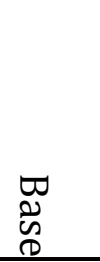 & 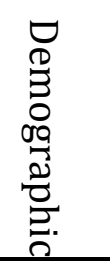 & 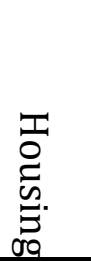 & $\begin{array}{l}\vec{D} \\
0 \\
0 \\
0 \\
0 \\
0 \\
\end{array}$ & $\begin{array}{l}T \\
D \\
\end{array}$ & 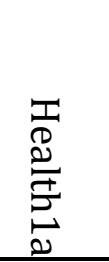 & 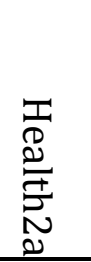 & 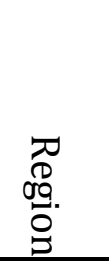 & 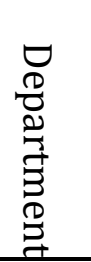 & $\mathrm{S}$ & $\mathrm{L}$ & $\mathrm{C}$ \\
\hline ems.5.014 & Yes & No & Yes & Yes & Yes & No & No & No & Yes & Yes & 412.7 & $1.56 \mathrm{E}+05$ & 386.3 \\
\hline ems.5.015 & Yes & Yes & Yes & Yes & Yes & No & No & No & Yes & Yes & 399.5 & $4.28 \mathrm{E}+03$ & 370.3 \\
\hline ems.5.016 & Yes & No & No & No & No & Yes & No & No & No & Yes & 390.9 & $2.78 \mathrm{E}+03$ & 390.8 \\
\hline ems.5.017 & Yes & Yes & No & No & No & Yes & No & No & Yes & Yes & 391.6 & $1.31 \mathrm{E}+05$ & 393.4 \\
\hline ems.5.018 & Yes & No & Yes & No & No & Yes & No & No & Yes & Yes & 1803.2 & $7.31 \mathrm{E}+03$ & 978.6 \\
\hline ems.5.019 & Yes & Yes & Yes & No & No & Yes & No & No & Yes & Yes & 927.4 & $1.09 \mathrm{E}+04$ & 678.7 \\
\hline ems.5.020 & Yes & No & No & Yes & No & Yes & No & No & Yes & Yes & 373.3 & $7.26 \mathrm{E}+02$ & 374.0 \\
\hline ems.5.021 & Yes & Yes & No & Yes & No & Yes & No & No & Yes & Yes & 373.6 & $6.02 \mathrm{E}+02$ & 373.9 \\
\hline ems.5.022 & Yes & No & Yes & Yes & No & Yes & No & No & Yes & Yes & 416.6 & $3.11 \mathrm{E}+04$ & 395.9 \\
\hline ems.5.023 & Yes & Yes & Yes & Yes & No & Yes & No & No & Yes & Yes & 461.9 & $2.92 \mathrm{E}+04$ & 416.1 \\
\hline ems.5.024 & Yes & No & No & No & Yes & Yes & No & No & No & Yes & 380.6 & $2.12 \mathrm{E}+03$ & 382.1 \\
\hline ems.5.025 & Yes & Yes & No & No & Yes & Yes & No & No & No & Yes & 377.3 & $5.76 \mathrm{E}+03$ & 374.8 \\
\hline ems.5.026 & Yes & No & Yes & No & Yes & Yes & No & No & Yes & Yes & 492.7 & $1.23 \mathrm{E}+04$ & 489.5 \\
\hline ems.5.027 & Yes & Yes & Yes & No & Yes & Yes & No & No & Yes & Yes & 377.9 & $1.59 \mathrm{E}+04$ & 378.7 \\
\hline ems.5.028 & Yes & No & No & Yes & Yes & Yes & No & No & Yes & Yes & 372.9 & $6.45 \mathrm{E}+02$ & 370.8 \\
\hline ems.5.029 & Yes & Yes & No & Yes & Yes & Yes & No & No & Yes & Yes & 372.8 & $9.05 \mathrm{E}+02$ & 370.8 \\
\hline ems.5.030 & Yes & No & Yes & Yes & Yes & Yes & No & No & Yes & Yes & 382.2 & $1.00 \mathrm{E}+04$ & 378.9 \\
\hline ems.5.031 & Yes & Yes & Yes & Yes & Yes & Yes & No & No & Yes & Yes & 377.3 & $2.25 \mathrm{E}+04$ & 369.1 \\
\hline ems.5.032 & Yes & No & No & No & No & No & Yes & No & No & Yes & 378.2 & $2.78 \mathrm{E}+25$ & 378.8 \\
\hline ems.5.033 & Yes & Yes & No & No & No & No & Yes & No & No & Yes & 373.2 & $4.36 \mathrm{E}+02$ & 371.3 \\
\hline ems.5.034 & Yes & No & Yes & No & No & No & Yes & No & Yes & Yes & 714.0 & $2.30 \mathrm{E}+03$ & 727.3 \\
\hline ems.5.035 & Yes & Yes & Yes & No & No & No & Yes & No & Yes & Yes & 443.4 & $8.03 \mathrm{E}+05$ & 495.2 \\
\hline ems.5.036 & Yes & No & No & Yes & No & No & Yes & No & No & Yes & 374.2 & $9.34 \mathrm{E}+02$ & 370.7 \\
\hline
\end{tabular}




\begin{tabular}{|c|c|c|c|c|c|c|c|c|c|c|c|c|c|}
\hline Model & $\begin{array}{l}\overleftrightarrow{\mathbb{8}} \\
\stackrel{\mathscr{2}}{7}\end{array}$ & 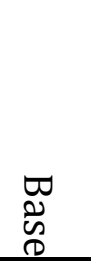 & 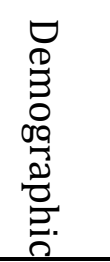 & 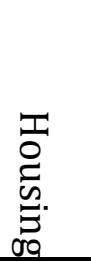 & 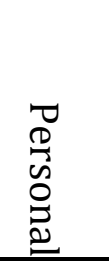 & $\begin{array}{l}\text { TI } \\
\text { D }\end{array}$ & 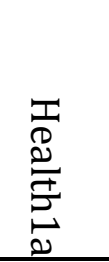 & 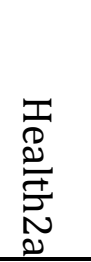 & 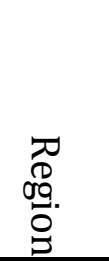 & 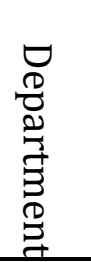 & $\mathrm{S}$ & $\mathrm{L}$ & $\mathrm{C}$ \\
\hline ems.5.037 & Yes & Yes & No & Yes & No & No & Yes & No & Yes & Yes & 373.5 & $7.91 \mathrm{E}+02$ & 371.2 \\
\hline ems.5.038 & Yes & No & Yes & Yes & No & No & Yes & No & Yes & Yes & 479.0 & $8.05 \mathrm{E}+03$ & 440.5 \\
\hline ems.5.039 & Yes & Yes & Yes & Yes & No & No & Yes & No & Yes & Yes & 495.3 & $3.94 \mathrm{E}+03$ & 484.3 \\
\hline ems.5.040 & Yes & No & No & No & Yes & No & Yes & No & Yes & Yes & 369.5 & $4.20 \mathrm{E}+02$ & 370.1 \\
\hline ems.5.041 & Yes & Yes & No & No & Yes & No & Yes & No & Yes & Yes & 368.5 & $6.58 \mathrm{E}+02$ & 369.0 \\
\hline ems.5.042 & Yes & No & Yes & No & Yes & No & Yes & No & Yes & Yes & 509.5 & $8.06 \mathrm{E}+04$ & 536.2 \\
\hline ems.5.043 & Yes & Yes & Yes & No & Yes & No & Yes & No & Yes & Yes & 397.8 & $2.86 \mathrm{E}+05$ & 412.4 \\
\hline ems.5.044 & Yes & No & No & Yes & Yes & No & Yes & No & No & Yes & 369.9 & $8.74 \mathrm{E}+02$ & 369.5 \\
\hline ems.5.045 & Yes & Yes & No & Yes & Yes & No & Yes & No & Yes & Yes & 368.6 & $1.25 \mathrm{E}+03$ & 369.1 \\
\hline ems.5.046 & Yes & No & Yes & Yes & Yes & No & Yes & No & Yes & Yes & 426.8 & $2.07 \mathrm{E}+03$ & 401.8 \\
\hline ems.5.047 & Yes & Yes & Yes & Yes & Yes & No & Yes & No & Yes & Yes & 409.2 & $6.57 \mathrm{E}+06$ & 387.5 \\
\hline ems.5.048 & Yes & No & No & No & No & Yes & Yes & No & Yes & Yes & 387.7 & $1.41 \mathrm{E}+03$ & 381.7 \\
\hline ems.5.049 & Yes & Yes & No & No & No & Yes & Yes & No & Yes & Yes & 385.3 & $9.32 \mathrm{E}+03$ & 381.8 \\
\hline ems.5.050 & Yes & No & Yes & No & No & Yes & Yes & No & Yes & Yes & 1619.2 & $9.25 \mathrm{E}+03$ & 926.3 \\
\hline ems.5.051 & Yes & Yes & Yes & No & No & Yes & Yes & No & Yes & Yes & 577.4 & $4.80 \mathrm{E}+03$ & 607.0 \\
\hline ems.5.052 & Yes & No & No & Yes & No & Yes & Yes & No & Yes & Yes & 372.2 & $5.12 \mathrm{E}+03$ & 373.4 \\
\hline ems.5.053 & Yes & Yes & No & Yes & No & Yes & Yes & No & Yes & Yes & 372.2 & $7.82 \mathrm{E}+02$ & 374.1 \\
\hline ems.5.054 & Yes & No & Yes & Yes & No & Yes & Yes & No & Yes & Yes & 432.5 & $2.12 \mathrm{E}+05$ & 418.8 \\
\hline ems.5.055 & Yes & Yes & Yes & Yes & No & Yes & Yes & No & Yes & Yes & 445.1 & $2.54 \mathrm{E}+05$ & 452.9 \\
\hline ems.5.056 & Yes & No & No & No & Yes & Yes & Yes & No & Yes & Yes & 378.9 & $7.79 \mathrm{E}+02$ & 376.3 \\
\hline ems.5.057 & Yes & Yes & No & No & Yes & Yes & Yes & No & Yes & Yes & 374.9 & $6.06 \mathrm{E}+02$ & 374.2 \\
\hline ems.5.058 & Yes & No & Yes & No & Yes & Yes & Yes & No & Yes & Yes & 636.6 & $7.45 \mathrm{E}+05$ & 484.5 \\
\hline ems.5.059 & Yes & Yes & Yes & No & Yes & Yes & Yes & No & Yes & Yes & 392.0 & $5.35 \mathrm{E}+04$ & 396.5 \\
\hline
\end{tabular}




\begin{tabular}{|c|c|c|c|c|c|c|c|c|c|c|c|c|c|}
\hline Model & $\begin{array}{l}\overleftrightarrow{\mathbb{8}} \\
\stackrel{\mathscr{2}}{7}\end{array}$ & 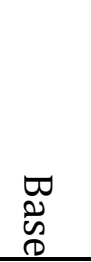 & 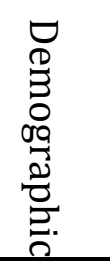 & 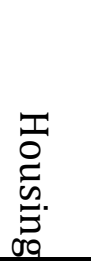 & $\begin{array}{l}\vec{D} \\
0 \\
0 \\
0 \\
0 \\
0 \\
\end{array}$ & $\begin{array}{l}\text { T. } \\
\text { हD } \\
\end{array}$ & 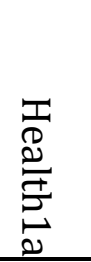 & 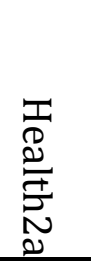 & 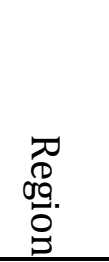 & 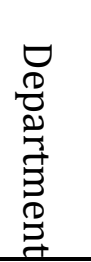 & $\mathrm{S}$ & $\mathrm{L}$ & $\mathrm{C}$ \\
\hline ems.5.060 & Yes & No & No & Yes & Yes & Yes & Yes & No & Yes & Yes & 367.6 & $5.61 \mathrm{E}+02$ & 371.0 \\
\hline ems.5.061 & Yes & Yes & No & Yes & Yes & Yes & Yes & No & Yes & Yes & 367.9 & $1.49 \mathrm{E}+03$ & 370.7 \\
\hline ems.5.062 & Yes & No & Yes & Yes & Yes & Yes & Yes & No & Yes & Yes & 385.1 & $6.91 \mathrm{E}+06$ & 392.8 \\
\hline ems.5.063 & Yes & Yes & Yes & Yes & Yes & Yes & Yes & No & Yes & Yes & 378.5 & $1.89 \mathrm{E}+07$ & 380.4 \\
\hline ems.5.064 & Yes & No & No & No & No & No & No & Yes & No & Yes & 381.9 & $1.34 \mathrm{E}+03$ & 382.2 \\
\hline ems.5.065 & Yes & Yes & No & No & No & No & No & Yes & Yes & Yes & 375.2 & $1.28 \mathrm{E}+03$ & 373.5 \\
\hline ems.5.066 & Yes & No & Yes & No & No & No & No & Yes & Yes & Yes & 579.5 & $2.70 \mathrm{E}+04$ & 608.8 \\
\hline ems.5.067 & Yes & Yes & Yes & No & No & No & No & Yes & Yes & Yes & 404.1 & $1.01 \mathrm{E}+09$ & 451.4 \\
\hline ems.5.068 & Yes & No & No & Yes & No & No & No & Yes & Yes & Yes & 375.4 & $7.57 \mathrm{E}+09$ & 385.8 \\
\hline ems.5.069 & Yes & Yes & No & Yes & No & No & No & Yes & Yes & Yes & 375.9 & $3.11 \mathrm{E}+06$ & 401.7 \\
\hline ems.5.070 & Yes & No & Yes & Yes & No & No & No & Yes & Yes & Yes & 483.4 & $7.52 \mathrm{E}+08$ & 428.1 \\
\hline ems.5.071 & Yes & Yes & Yes & Yes & No & No & No & Yes & Yes & Yes & 492.6 & $6.26 \mathrm{E}+09$ & 457.7 \\
\hline ems.5.072 & Yes & No & No & No & Yes & No & No & Yes & No & Yes & 369.3 & $4.76 \mathrm{E}+03$ & 375.9 \\
\hline ems.5.073 & Yes & Yes & No & No & Yes & No & No & Yes & Yes & Yes & 368.8 & $3.41 \mathrm{E}+06$ & 374.9 \\
\hline ems.5.074 & Yes & No & Yes & No & Yes & No & No & Yes & Yes & Yes & 503.5 & $4.79 \mathrm{E}+04$ & 507.1 \\
\hline ems.5.075 & Yes & Yes & Yes & No & Yes & No & No & Yes & Yes & Yes & 398.1 & $3.38 \mathrm{E}+03$ & 420.2 \\
\hline ems.5.076 & Yes & No & No & Yes & Yes & No & No & Yes & Yes & Yes & 370.0 & $1.60 \mathrm{E}+06$ & 375.4 \\
\hline ems.5.077 & Yes & Yes & No & Yes & Yes & No & No & Yes & Yes & Yes & 368.7 & $8.92 \mathrm{E}+07$ & 390.1 \\
\hline ems.5.078 & Yes & No & Yes & Yes & Yes & No & No & Yes & Yes & Yes & 447.3 & $1.20 \mathrm{E}+09$ & 396.0 \\
\hline ems.5.079 & Yes & Yes & Yes & Yes & Yes & No & No & Yes & Yes & Yes & 426.9 & $2.12 \mathrm{E}+10$ & 387.1 \\
\hline ems.5.080 & Yes & No & No & No & No & Yes & No & Yes & No & Yes & 382.1 & $4.20 \mathrm{E}+02$ & 382.0 \\
\hline ems.5.081 & Yes & Yes & No & No & No & Yes & No & Yes & Yes & Yes & 379.0 & $4.02 \mathrm{E}+02$ & 379.2 \\
\hline ems.5.082 & Yes & No & Yes & No & No & Yes & No & Yes & Yes & Yes & 1137.6 & $1.15 \mathrm{E}+04$ & 632.3 \\
\hline
\end{tabular}




\begin{tabular}{|c|c|c|c|c|c|c|c|c|c|c|c|c|c|}
\hline Model & $\begin{array}{l}\overleftrightarrow{\mathbb{8}} \\
\stackrel{\mathscr{2}}{7}\end{array}$ & 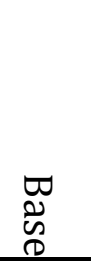 & 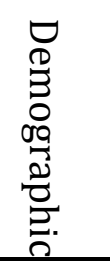 & 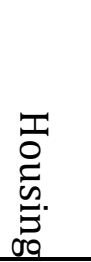 & 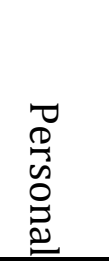 & $\begin{array}{l}\text { T. } \\
\text { D } \\
\end{array}$ & 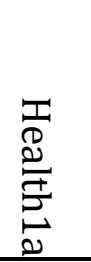 & 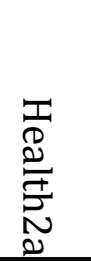 & 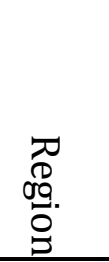 & 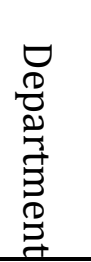 & $\mathrm{S}$ & $\mathrm{L}$ & $\mathrm{C}$ \\
\hline ems.5.083 & Yes & Yes & Yes & No & No & Yes & No & Yes & Yes & Yes & 471.5 & $1.80 \mathrm{E}+04$ & 492.9 \\
\hline ems.5.084 & Yes & No & No & Yes & No & Yes & No & Yes & Yes & Yes & 373.9 & $7.27 \mathrm{E}+03$ & 381.2 \\
\hline ems.5.085 & Yes & Yes & No & Yes & No & Yes & No & Yes & Yes & Yes & 375.6 & $9.72 \mathrm{E}+03$ & 391.3 \\
\hline ems.5.086 & Yes & No & Yes & Yes & No & Yes & No & Yes & Yes & Yes & 423.8 & $4.67 \mathrm{E}+05$ & 396.6 \\
\hline ems.5.087 & Yes & Yes & Yes & Yes & No & Yes & No & Yes & Yes & Yes & 438.6 & $6.07 \mathrm{E}+04$ & 426.6 \\
\hline ems.5.088 & Yes & No & No & No & Yes & Yes & No & Yes & Yes & Yes & 371.3 & $4.43 \mathrm{E}+02$ & 380.8 \\
\hline ems.5.089 & Yes & Yes & No & No & Yes & Yes & No & Yes & No & Yes & 370.4 & $4.61 \mathrm{E}+02$ & 380.1 \\
\hline ems.5.090 & Yes & No & Yes & No & Yes & Yes & No & Yes & Yes & Yes & 566.0 & $1.63 \mathrm{E}+05$ & 438.8 \\
\hline ems.5.091 & Yes & Yes & Yes & No & Yes & Yes & No & Yes & Yes & Yes & 388.9 & $1.62 \mathrm{E}+06$ & 392.3 \\
\hline ems.5.092 & Yes & No & No & Yes & Yes & Yes & No & Yes & Yes & Yes & 366.5 & $2.07 \mathrm{E}+04$ & 374.4 \\
\hline ems.5.093 & Yes & Yes & No & Yes & Yes & Yes & No & Yes & Yes & Yes & 367.9 & $8.73 \mathrm{E}+04$ & 383.7 \\
\hline ems.5.094 & Yes & No & Yes & Yes & Yes & Yes & No & Yes & Yes & Yes & 390.4 & $8.83 \mathrm{E}+09$ & 381.7 \\
\hline ems.5.095 & Yes & Yes & Yes & Yes & Yes & Yes & No & Yes & Yes & Yes & 385.6 & $1.98 \mathrm{E}+06$ & 375.6 \\
\hline ems.5.096 & Yes & No & No & No & No & No & Yes & Yes & Yes & Yes & 378.2 & $8.09 \mathrm{E}+19$ & 380.0 \\
\hline ems.5.097 & Yes & Yes & No & No & No & No & Yes & Yes & No & Yes & 370.3 & $2.92 \mathrm{E}+19$ & 371.1 \\
\hline ems.5.098 & Yes & No & Yes & No & No & No & Yes & Yes & Yes & Yes & 543.1 & $3.59 \mathrm{E}+25$ & 543.2 \\
\hline ems.5.099 & Yes & Yes & Yes & No & No & No & Yes & Yes & Yes & Yes & 410.6 & $4.10 \mathrm{E}+15$ & 436.9 \\
\hline ems.5.100 & Yes & No & No & Yes & No & No & Yes & Yes & Yes & Yes & 373.8 & $2.47 \mathrm{E}+08$ & 370.5 \\
\hline ems.5.101 & Yes & Yes & No & Yes & No & No & Yes & Yes & Yes & Yes & 373.6 & $7.05 \mathrm{E}+16$ & 374.4 \\
\hline ems.5.102 & Yes & No & Yes & Yes & No & No & Yes & Yes & Yes & Yes & 456.2 & $\operatorname{Inf}$ & 412.0 \\
\hline ems.5.103 & Yes & Yes & Yes & Yes & No & No & Yes & Yes & Yes & Yes & 488.0 & $3.32 \mathrm{E}+19$ & 451.0 \\
\hline ems.5.104 & Yes & No & No & No & Yes & No & Yes & Yes & No & Yes & 365.3 & $1.68 \mathrm{E}+18$ & 366.5 \\
\hline ems.5.105 & Yes & Yes & No & No & Yes & No & Yes & Yes & Yes & Yes & 364.6 & $2.06 \mathrm{E}+32$ & 365.3 \\
\hline
\end{tabular}




\begin{tabular}{|c|c|c|c|c|c|c|c|c|c|c|c|c|c|}
\hline Model & 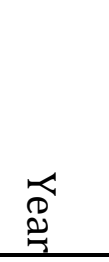 & 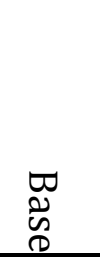 & 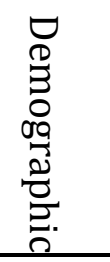 & 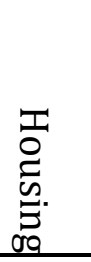 & $\begin{array}{l}\vec{J} \\
0 \\
0 \\
0 \\
0 \\
\\
\end{array}$ & $\begin{array}{l}7 \\
D \\
\end{array}$ & 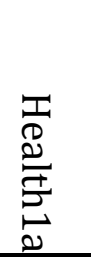 & 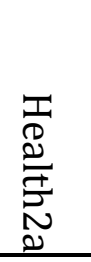 & 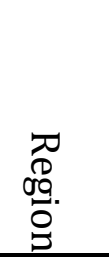 & 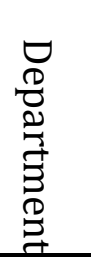 & $\mathrm{S}$ & $\mathrm{L}$ & $\mathrm{C}$ \\
\hline ems.5.106 & Yes & No & Yes & No & Yes & No & Yes & Yes & Yes & Yes & 496.5 & $7.75 \mathrm{E}+31$ & 467.2 \\
\hline ems.5.107 & Yes & Yes & Yes & No & Yes & No & Yes & Yes & Yes & Yes & 406.1 & $\operatorname{Inf}$ & 413.4 \\
\hline ems.5.108 & Yes & No & No & Yes & Yes & No & Yes & Yes & Yes & Yes & 367.7 & $2.73 \mathrm{E}+11$ & 366.7 \\
\hline ems.5.109 & Yes & Yes & No & Yes & Yes & No & Yes & Yes & Yes & Yes & 366.5 & $1.09 \mathrm{E}+52$ & 367.2 \\
\hline ems.5.110 & Yes & No & Yes & Yes & Yes & No & Yes & Yes & Yes & Yes & 432.3 & $4.64 \mathrm{E}+16$ & 385.6 \\
\hline ems.5.111 & Yes & Yes & Yes & Yes & Yes & No & Yes & Yes & Yes & Yes & 431.8 & $3.20 \mathrm{E}+28$ & 389.2 \\
\hline ems.5.112 & Yes & No & No & No & No & Yes & Yes & Yes & Yes & Yes & 378.0 & $6.50 \mathrm{E}+06$ & 378.5 \\
\hline ems.5.113 & Yes & Yes & No & No & No & Yes & Yes & Yes & Yes & Yes & 373.2 & $2.11 \mathrm{E}+06$ & 376.9 \\
\hline ems.5.114 & Yes & No & Yes & No & No & Yes & Yes & Yes & Yes & Yes & 816.9 & $4.27 \mathrm{E}+05$ & 541.4 \\
\hline ems.5.115 & Yes & Yes & Yes & No & No & Yes & Yes & Yes & Yes & Yes & 445.4 & $3.24 \mathrm{E}+05$ & 455.0 \\
\hline ems.5.116 & Yes & No & No & Yes & No & Yes & Yes & Yes & Yes & Yes & 369.1 & $1.29 \mathrm{E}+07$ & 372.0 \\
\hline ems.5.117 & Yes & Yes & No & Yes & No & Yes & Yes & Yes & Yes & Yes & 369.3 & $6.85 \mathrm{E}+06$ & 378.3 \\
\hline ems.5.118 & Yes & No & Yes & Yes & No & Yes & Yes & Yes & No & Yes & 400.0 & $2.13 \mathrm{E}+11$ & 389.8 \\
\hline ems.5.119 & Yes & Yes & Yes & Yes & No & Yes & Yes & Yes & No & Yes & 417.4 & $2.68 \mathrm{E}+13$ & 416.8 \\
\hline ems.5.120 & Yes & No & No & No & Yes & Yes & Yes & Yes & Yes & Yes & 366.8 & $1.02 \mathrm{E}+05$ & 369.2 \\
\hline ems.5.121 & Yes & Yes & No & No & Yes & Yes & Yes & Yes & Yes & Yes & 365.7 & $5.78 \mathrm{E}+05$ & 368.4 \\
\hline ems.5.122 & Yes & No & Yes & No & Yes & Yes & Yes & Yes & Yes & Yes & 508.7 & $6.77 \mathrm{E}+05$ & 418.0 \\
\hline ems.5.123 & Yes & Yes & Yes & No & Yes & Yes & Yes & Yes & No & Yes & 385.3 & $1.27 \mathrm{E}+10$ & 389.3 \\
\hline ems.5.124 & Yes & No & No & Yes & Yes & Yes & Yes & Yes & Yes & Yes & 362.9 & $1.39 \mathrm{E}+04$ & 368.7 \\
\hline ems.5.125 & Yes & Yes & No & Yes & Yes & Yes & Yes & Yes & Yes & Yes & 363.4 & $2.89 \mathrm{E}+04$ & 370.0 \\
\hline ems.5.126 & Yes & No & Yes & Yes & Yes & Yes & Yes & Yes & Yes & Yes & 378.2 & $6.63 \mathrm{E}+09$ & 379.2 \\
\hline ems.5.127 & Yes & Yes & Yes & Yes & Yes & Yes & Yes & Yes & Yes & Yes & 379.2 & $1.97 \mathrm{E}+12$ & 378.2 \\
\hline
\end{tabular}


Table 27:RMS Errors of the models for EMS risk based on the county health data set.

Model(s) with the lowest RMS Error are in bold. A blank field indicates that the relevant model was not run.

\begin{tabular}{|c|c|c|c|c|c|c|c|c|c|c|c|c|c|c|c|}
\hline Model & $\begin{array}{l}\underset{\mathbb{0}}{\approx} \\
\stackrel{7}{2}\end{array}$ & 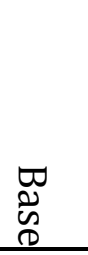 & 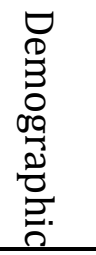 & 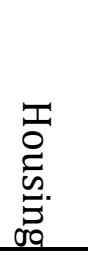 & $\begin{array}{l}0 \\
0 \\
0 \\
0 \\
0 \\
0 \\
0 \\
\end{array}$ & $\begin{array}{l}\text { Tै } \\
\text { D. } \\
\end{array}$ & 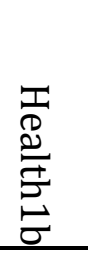 & 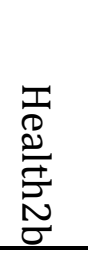 & 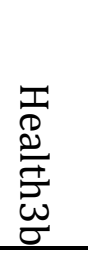 & 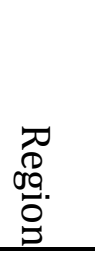 & 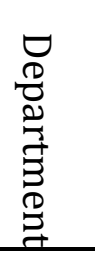 & $\mathrm{S}$ & $\mathrm{L}$ & $\mathrm{C}$ & Notes \\
\hline ems.C.const & No & No & No & No & No & No & No & No & No & No & No & 386.7 & & & \\
\hline ems.C.fx & No & No & No & No & No & No & No & No & No & No & Yes & 356.2 & & & \\
\hline ems.C.lasso.min & Yes & Yes & Yes & Yes & Yes & Yes & Yes & Yes & Yes & Yes & No & 348.0 & & & \\
\hline ems.C.lasso.1se & Yes & Yes & Yes & Yes & Yes & Yes & Yes & Yes & Yes & Yes & No & 360.1 & & & \\
\hline ems.C.rf & Yes & Yes & Yes & Yes & Yes & Yes & Yes & Yes & Yes & Yes & No & 330.1 & & & \\
\hline ems.C.000 & Yes & No & No & No & No & No & No & No & No & No & Yes & 356.2 & $3.56 \mathrm{E}+02$ & 356.4 & \\
\hline ems.C.001 & Yes & Yes & No & No & No & No & No & No & No & No & Yes & 351.3 & $3.50 \mathrm{E}+02$ & 350.8 & \\
\hline ems.C.002 & Yes & No & Yes & No & No & No & No & No & No & No & Yes & 436.5 & $4.07 \mathrm{E}+02$ & 397.3 & \\
\hline ems.C.003 & Yes & Yes & Yes & No & No & No & No & No & No & No & Yes & 377.6 & $3.75 E+02$ & 360.7 & \\
\hline ems.C.004 & Yes & No & No & Yes & No & No & No & No & No & No & Yes & 546.9 & $3.41 \mathrm{E}+02$ & 336.3 & \\
\hline ems.C.005 & Yes & Yes & No & Yes & No & No & No & No & No & No & Yes & 563.0 & $3.39 E+02$ & 335.7 & \\
\hline ems.C.006 & Yes & No & Yes & Yes & No & No & No & No & No & No & Yes & 485.1 & $3.48 \mathrm{E}+02$ & 343.5 & \\
\hline ems.C.007 & Yes & Yes & Yes & Yes & No & No & No & No & No & Yes & Yes & 483.5 & $3.63 E+02$ & 342.8 & \\
\hline ems.C.008 & Yes & No & No & No & Yes & No & No & No & No & No & Yes & 335.6 & $3.37 \mathrm{E}+02$ & 336.2 & \\
\hline ems.C.009 & Yes & Yes & No & No & Yes & No & No & No & No & No & Yes & 335.0 & $3.35 E+02$ & 335.3 & \\
\hline ems.C.010 & Yes & No & Yes & No & Yes & No & No & No & No & No & Yes & 341.2 & $3.66 \mathrm{E}+02$ & 348.0 & \\
\hline ems.C.011 & Yes & Yes & Yes & No & Yes & No & No & No & No & No & Yes & 333.6 & $3.51 \mathrm{E}+02$ & 332.2 & \\
\hline ems.C.012 & Yes & No & No & Yes & Yes & No & No & No & No & No & Yes & 369.8 & $3.44 \mathrm{E}+02$ & 332.6 & \\
\hline ems.C.013 & Yes & Yes & No & Yes & Yes & No & No & No & No & No & Yes & 365.9 & $3.49 \mathrm{E}+02$ & 330.7 & \\
\hline ems.C.014 & Yes & No & Yes & Yes & Yes & No & No & No & No & Yes & Yes & 400.2 & $3.57 \mathrm{E}+02$ & 342.2 & \\
\hline ems.C.015 & Yes & Yes & Yes & Yes & Yes & No & No & No & No & Yes & Yes & 386.7 & $3.58 \mathrm{E}+02$ & 345.5 & \\
\hline
\end{tabular}




\begin{tabular}{|c|c|c|c|c|c|c|c|c|c|c|c|c|c|c|c|}
\hline Model & $\underset{\mathbb{d}}{\stackrel{2}{2}}$ & 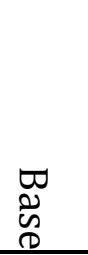 & 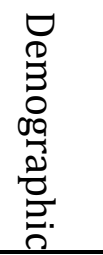 & 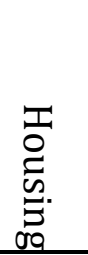 & 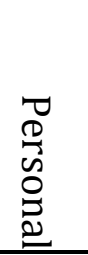 & D & 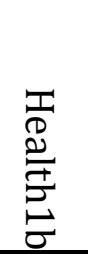 & 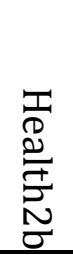 & 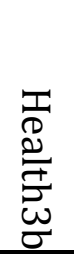 & 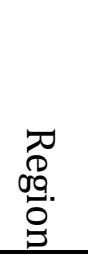 & 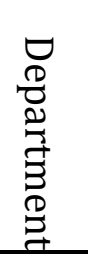 & $\mathrm{S}$ & $\mathrm{L}$ & $\mathrm{C}$ & Notes \\
\hline ems.C.016 & Yes & No & No & No & No & Yes & No & No & No & No & Yes & 353.4 & $3.82 \mathrm{E}+02$ & 352.8 & \\
\hline ems.C.017 & Yes & Yes & No & No & No & Yes & No & No & No & No & Yes & 351.9 & $3.77 \mathrm{E}+02$ & 350.8 & \\
\hline ems.C.018 & Yes & No & Yes & No & No & Yes & No & No & No & Yes & Yes & 1025.0 & $6.85 \mathrm{E}+02$ & 650.8 & \\
\hline ems.C.019 & Yes & Yes & Yes & No & No & Yes & No & No & No & Yes & Yes & 539.9 & $5.43 \mathrm{E}+02$ & 435.2 & \\
\hline ems.C.020 & Yes & No & No & Yes & No & Yes & No & No & No & No & Yes & 521.7 & $3.46 \mathrm{E}+02$ & 336.5 & \\
\hline ems.C.021 & Yes & Yes & No & Yes & No & Yes & No & No & No & No & Yes & 533.2 & $3.45 E+02$ & 336.2 & \\
\hline ems.C.022 & Yes & No & Yes & Yes & No & Yes & No & No & No & No & Yes & 462.9 & $3.66 \mathrm{E}+02$ & 338.2 & \\
\hline ems.C.023 & Yes & Yes & Yes & Yes & No & Yes & No & No & No & Yes & Yes & 457.2 & $3.97 \mathrm{E}+02$ & 338.7 & \\
\hline ems.C.024 & Yes & No & No & No & Yes & Yes & No & No & No & No & Yes & 339.6 & $3.67 \mathrm{E}+02$ & 337.0 & \\
\hline ems.C.025 & Yes & Yes & No & No & Yes & Yes & No & No & No & No & Yes & 338.9 & $3.59 \mathrm{E}+02$ & 335.4 & \\
\hline ems.C.026 & Yes & No & Yes & No & Yes & Yes & No & No & No & No & Yes & 381.4 & $4.35 \mathrm{E}+02$ & 373.5 & \\
\hline ems.C.027 & Yes & Yes & Yes & No & Yes & Yes & No & No & No & No & Yes & 335.9 & $3.81 \mathrm{E}+02$ & 331.7 & \\
\hline ems.C.028 & Yes & No & No & Yes & Yes & Yes & No & No & No & No & Yes & 374.8 & $3.62 \mathrm{E}+02$ & 331.6 & \\
\hline ems.C.029 & Yes & Yes & No & Yes & Yes & Yes & No & No & No & Yes & Yes & 375.2 & $3.65 E+02$ & 329.9 & \\
\hline ems.C.030 & Yes & No & Yes & Yes & Yes & Yes & No & No & No & Yes & Yes & 400.6 & $3.97 \mathrm{E}+02$ & 337.0 & \\
\hline ems.C.031 & Yes & Yes & Yes & Yes & Yes & Yes & No & No & No & Yes & Yes & 389.2 & $3.89 \mathrm{E}+02$ & 329.0 & \\
\hline ems.C.032 & Yes & No & No & No & No & No & Yes & No & No & No & Yes & 356.1 & $2.70 \mathrm{E}+03$ & 343.1 & $*$ \\
\hline ems.C.033 & Yes & Yes & No & No & No & No & Yes & No & No & No & Yes & 351.2 & $1.39 \mathrm{E}+06$ & 338.0 & $*$ \\
\hline ems.C.034 & Yes & No & Yes & No & No & No & Yes & No & No & Yes & Yes & 443.7 & $1.59 \mathrm{E}+04$ & 384.3 & $*$ \\
\hline ems.C.035 & Yes & Yes & Yes & No & No & No & Yes & No & No & Yes & Yes & 384.8 & $2.85 E+06$ & 351.7 & $*$ \\
\hline ems.C.036 & Yes & No & No & Yes & No & No & Yes & No & No & No & Yes & 551.5 & $1.92 \mathrm{E}+05$ & 321.7 & $*$ \\
\hline ems.C.037 & Yes & Yes & No & Yes & No & No & Yes & No & No & No & Yes & 565.8 & $3.25 \mathrm{E}+06$ & 321.9 & $*$ \\
\hline ems.C.038 & Yes & No & Yes & Yes & No & No & Yes & No & No & Yes & Yes & 508.6 & $2.62 \mathrm{E}+05$ & 328.2 & $*$ \\
\hline
\end{tabular}




\begin{tabular}{|c|c|c|c|c|c|c|c|c|c|c|c|c|c|c|c|}
\hline Model & 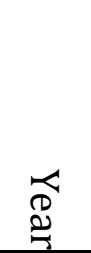 & 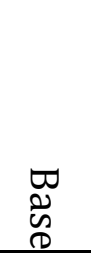 & 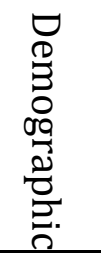 & 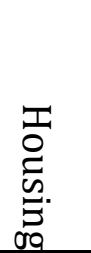 & $\begin{array}{l}\vec{D} \\
0 \\
0 \\
0 \\
0 \\
0\end{array}$ & $\begin{array}{l}T \\
\text { DD }\end{array}$ & 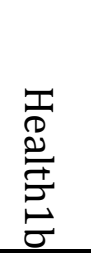 & 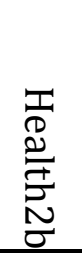 & 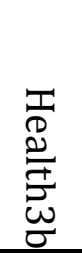 & 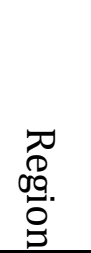 & 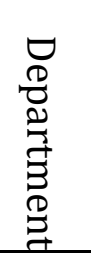 & $\mathrm{S}$ & $\mathrm{L}$ & $\mathrm{C}$ & Notes \\
\hline ems.C.039 & Yes & Yes & Yes & Yes & No & No & Yes & No & No & No & Yes & 515.1 & $8.68 \mathrm{E}+07$ & 331.8 & $*$ \\
\hline ems.C.040 & Yes & No & No & No & Yes & No & Yes & No & No & No & Yes & 335.6 & $6.88 \mathrm{E}+05$ & 325.1 & * \\
\hline ems.C.041 & Yes & Yes & No & No & Yes & No & Yes & No & No & No & Yes & 335.1 & $4.39 \mathrm{E}+07$ & 323.8 & $*$ \\
\hline ems.C.042 & Yes & No & Yes & No & Yes & No & Yes & No & No & No & Yes & 340.3 & $1.06 \mathrm{E}+06$ & 329.5 & * \\
\hline ems.C.043 & Yes & Yes & Yes & No & Yes & No & Yes & No & No & Yes & Yes & 334.2 & $3.38 \mathrm{E}+07$ & 318.6 & * \\
\hline ems.C.044 & Yes & No & No & Yes & Yes & No & Yes & No & No & No & Yes & 371.0 & $6.62 \mathrm{E}+05$ & 316.2 & * \\
\hline ems.C.045 & Yes & Yes & No & Yes & Yes & No & Yes & No & No & No & Yes & 366.7 & $1.32 \mathrm{E}+08$ & 317.1 & * \\
\hline ems.C.046 & Yes & No & Yes & Yes & Yes & No & Yes & No & No & Yes & Yes & 404.8 & $1.28 \mathrm{E}+06$ & 312.1 & * \\
\hline ems.C.047 & Yes & Yes & Yes & Yes & Yes & No & Yes & No & No & Yes & Yes & 390.7 & $2.36 \mathrm{E}+09$ & 311.2 & $*$ \\
\hline ems.C.048 & Yes & No & No & No & No & Yes & Yes & No & No & No & Yes & 353.8 & $2.08 \mathrm{E}+03$ & 338.9 & * \\
\hline ems.C.049 & Yes & Yes & No & No & No & Yes & Yes & No & No & No & Yes & 352.0 & $2.26 \mathrm{E}+05$ & 337.3 & * \\
\hline ems.C.050 & Yes & No & Yes & No & No & Yes & Yes & No & No & No & Yes & 977.3 & $6.01 \mathrm{E}+03$ & 499.1 & * \\
\hline ems.C.051 & Yes & Yes & Yes & No & No & Yes & Yes & No & No & Yes & Yes & 552.6 & $3.14 \mathrm{E}+05$ & 387.1 & * \\
\hline ems.C.052 & Yes & No & No & Yes & No & Yes & Yes & No & No & No & Yes & 532.4 & $3.04 \mathrm{E}+04$ & 321.7 & * \\
\hline ems.C.053 & Yes & Yes & No & Yes & No & Yes & Yes & No & No & No & Yes & 543.0 & $1.37 \mathrm{E}+05$ & 322.0 & * \\
\hline ems.C.054 & Yes & No & Yes & Yes & No & Yes & Yes & No & No & Yes & Yes & 476.3 & $1.66 \mathrm{E}+05$ & 319.8 & * \\
\hline ems.C.055 & Yes & Yes & Yes & Yes & No & Yes & Yes & No & No & Yes & Yes & 478.3 & $8.51 \mathrm{E}+06$ & 322.0 & * \\
\hline ems.C.056 & Yes & No & No & No & Yes & Yes & Yes & No & No & No & Yes & 337.2 & $5.37 \mathrm{E}+04$ & 324.2 & $*$ \\
\hline ems.C.057 & Yes & Yes & No & No & Yes & Yes & Yes & No & No & No & Yes & 336.7 & $1.20 \mathrm{E}+06$ & 323.8 & * \\
\hline ems.C.058 & Yes & No & Yes & No & Yes & Yes & Yes & No & No & Yes & Yes & 366.2 & $3.45 \mathrm{E}+05$ & 334.1 & * \\
\hline ems.C.059 & Yes & Yes & Yes & No & Yes & Yes & Yes & No & No & No & Yes & 335.9 & $3.75 E+06$ & 315.9 & * \\
\hline ems.C.060 & Yes & No & No & Yes & Yes & Yes & Yes & No & No & No & Yes & 377.3 & $1.72 \mathrm{E}+05$ & 315.9 & * \\
\hline ems.C.061 & Yes & Yes & No & Yes & Yes & Yes & Yes & No & No & No & Yes & 377.0 & $5.38 \mathrm{E}+06$ & 316.8 & * \\
\hline
\end{tabular}




\begin{tabular}{|c|c|c|c|c|c|c|c|c|c|c|c|c|c|c|c|}
\hline Model & 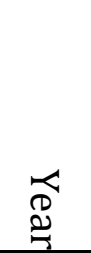 & 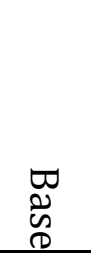 & 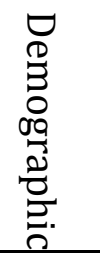 & 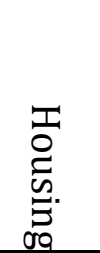 & $\begin{array}{l}\overrightarrow{0} \\
0 \\
0 \\
0 \\
0 \\
0\end{array}$ & $\underset{D}{T}$ & 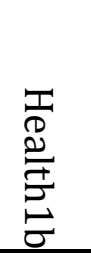 & 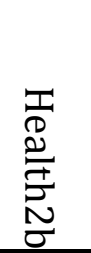 & 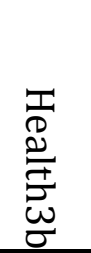 & 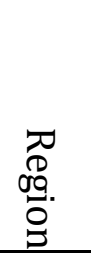 & 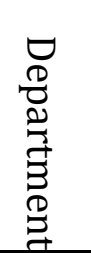 & $\mathrm{S}$ & $\mathrm{L}$ & $\mathrm{C}$ & Notes \\
\hline ems.C.062 & Yes & No & Yes & Yes & Yes & Yes & Yes & No & No & Yes & Yes & 403.9 & $8.55 \mathrm{E}+05$ & 310.9 & $*$ \\
\hline ems.C.063 & Yes & Yes & Yes & Yes & Yes & Yes & Yes & No & No & Yes & Yes & 392.5 & $2.07 \mathrm{E}+08$ & 310.8 & * \\
\hline ems.C.064 & Yes & No & No & No & No & No & No & Yes & No & No & Yes & 356.3 & $9.56 \mathrm{E}+06$ & 342.4 & $*$ \\
\hline ems.C.065 & Yes & Yes & No & No & No & No & No & Yes & No & No & Yes & 351.3 & $2.34 \mathrm{E}+07$ & 336.6 & * \\
\hline ems.C.066 & Yes & No & Yes & No & No & No & No & Yes & No & Yes & Yes & 444.3 & $1.83 \mathrm{E}+08$ & 383.6 & * \\
\hline ems.C.067 & Yes & Yes & Yes & No & No & No & No & Yes & No & Yes & Yes & 385.2 & $2.01 \mathrm{E}+08$ & 351.1 & * \\
\hline ems.C.068 & Yes & No & No & Yes & No & No & No & Yes & No & No & Yes & 542.8 & $4.97 \mathrm{E}+07$ & 320.9 & * \\
\hline ems.C.069 & Yes & Yes & No & Yes & No & No & No & Yes & No & No & Yes & 557.6 & $7.52 \mathrm{E}+07$ & 321.0 & * \\
\hline ems.C.070 & Yes & No & Yes & Yes & No & No & No & Yes & No & No & Yes & 510.3 & $1.08 \mathrm{E}+08$ & 327.6 & $*$ \\
\hline ems.C.071 & Yes & Yes & Yes & Yes & No & No & No & Yes & No & Yes & Yes & 517.4 & $1.24 \mathrm{E}+08$ & 331.3 & * \\
\hline ems.C.072 & Yes & No & No & No & Yes & No & No & Yes & No & No & Yes & 335.6 & $8.50 \mathrm{E}+07$ & 321.3 & * \\
\hline ems.C.073 & Yes & Yes & No & No & Yes & No & No & Yes & No & No & Yes & 335.0 & $1.60 \mathrm{E}+08$ & 320.5 & * \\
\hline ems.C.074 & Yes & No & Yes & No & Yes & No & No & Yes & No & Yes & Yes & 340.7 & $1.71 \mathrm{E}+08$ & 329.2 & * \\
\hline ems.C.075 & Yes & Yes & Yes & No & Yes & No & No & Yes & No & Yes & Yes & 334.3 & $1.87 \mathrm{E}+08$ & 318.3 & * \\
\hline ems.C.076 & Yes & No & No & Yes & Yes & No & No & Yes & No & No & Yes & 371.5 & $8.63 \mathrm{E}+07$ & 315.1 & * \\
\hline ems.C.077 & Yes & Yes & No & Yes & Yes & No & No & Yes & No & No & Yes & 367.2 & $1.34 \mathrm{E}+08$ & 314.6 & * \\
\hline ems.C.078 & Yes & No & Yes & Yes & Yes & No & No & Yes & No & Yes & Yes & 406.9 & $1.20 \mathrm{E}+08$ & 311.9 & * \\
\hline ems.C.079 & Yes & Yes & Yes & Yes & Yes & No & No & Yes & No & Yes & Yes & 392.6 & $1.46 \mathrm{E}+08$ & 310.9 & * \\
\hline ems.C.080 & Yes & No & No & No & No & Yes & No & Yes & No & No & Yes & 353.6 & $1.07 \mathrm{E}+07$ & 337.9 & * \\
\hline ems.C.081 & Yes & Yes & No & No & No & Yes & No & Yes & No & No & Yes & 351.8 & $2.20 \mathrm{E}+07$ & 336.2 & * \\
\hline ems.C.082 & Yes & No & Yes & No & No & Yes & No & Yes & No & Yes & Yes & 919.0 & $6.80 \mathrm{E}+07$ & 498.4 & * \\
\hline ems.C.083 & Yes & Yes & Yes & No & No & Yes & No & Yes & No & Yes & Yes & 529.2 & $8.24 \mathrm{E}+07$ & 386.4 & * \\
\hline ems.C.084 & Yes & No & No & Yes & No & Yes & No & Yes & No & No & Yes & 521.2 & $2.52 \mathrm{E}+07$ & 320.9 & $*$ \\
\hline
\end{tabular}




\begin{tabular}{|c|c|c|c|c|c|c|c|c|c|c|c|c|c|c|c|}
\hline Model & $\underset{\mathbb{d}}{\stackrel{2}{2}}$ & 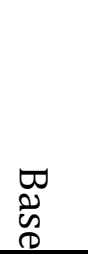 & 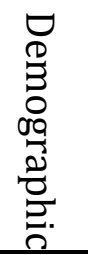 & 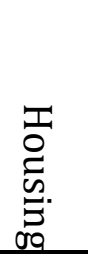 & $\begin{array}{l}\vec{D} \\
\mathbb{D} \\
\vec{D} \\
0 \\
0 \\
0 \\
D\end{array}$ & D & 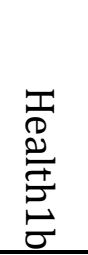 & 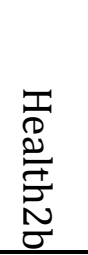 & 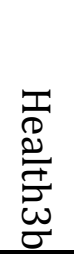 & 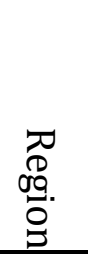 & 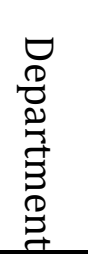 & $\mathrm{S}$ & $\mathrm{L}$ & $\mathrm{C}$ & Notes \\
\hline ems.C.085 & Yes & Yes & No & Yes & No & Yes & No & Yes & No & No & Yes & 531.7 & $3.93 \mathrm{E}+07$ & 321.1 & $*$ \\
\hline ems.C.086 & Yes & No & Yes & Yes & No & Yes & No & Yes & No & Yes & Yes & 474.6 & $5.63 \mathrm{E}+07$ & 319.3 & $*$ \\
\hline ems.C.087 & Yes & Yes & Yes & Yes & No & Yes & No & Yes & No & Yes & Yes & 475.1 & $6.80 \mathrm{E}+07$ & 321.4 & $*$ \\
\hline ems.C.088 & Yes & No & No & No & Yes & Yes & No & Yes & No & No & Yes & 337.3 & $4.27 \mathrm{E}+07$ & 320.9 & $*$ \\
\hline ems.C.089 & Yes & Yes & No & No & Yes & Yes & No & Yes & No & No & Yes & 336.8 & $7.81 \mathrm{E}+07$ & 320.3 & $*$ \\
\hline ems.C.090 & Yes & No & Yes & No & Yes & Yes & No & Yes & No & No & Yes & 366.7 & $8.56 \mathrm{E}+07$ & 333.9 & $*$ \\
\hline ems.C.091 & Yes & Yes & Yes & No & Yes & Yes & No & Yes & No & No & Yes & 335.9 & $8.68 E+07$ & 315.6 & $*$ \\
\hline ems.C.092 & Yes & No & No & Yes & Yes & Yes & No & Yes & No & No & Yes & 376.7 & $4.65 E+07$ & 315.0 & $*$ \\
\hline ems.C.093 & Yes & Yes & No & Yes & Yes & Yes & No & Yes & No & No & Yes & 376.8 & $6.84 \mathrm{E}+07$ & 314.5 & $*$ \\
\hline ems.C.094 & Yes & No & Yes & Yes & Yes & Yes & No & Yes & No & No & Yes & 404.5 & $6.22 \mathrm{E}+07$ & 310.8 & $*$ \\
\hline ems.C.095 & Yes & Yes & Yes & Yes & Yes & Yes & No & Yes & No & Yes & Yes & 393.0 & $7.63 \mathrm{E}+07$ & 310.6 & $*$ \\
\hline ems.C.096 & Yes & No & No & No & No & No & Yes & Yes & No & No & Yes & 356.2 & $1.56 \mathrm{E}+07$ & 356.7 & $*$ \\
\hline ems.C.097 & Yes & Yes & No & No & No & No & Yes & Yes & No & No & Yes & 351.3 & $1.25 \mathrm{E}+07$ & 430.1 & $*$ \\
\hline ems.C.098 & Yes & No & Yes & No & No & No & Yes & Yes & No & Yes & Yes & 446.6 & $2.04 \mathrm{E}+07$ & 444.2 & $*$ \\
\hline ems.C.099 & Yes & Yes & Yes & No & No & No & Yes & Yes & No & Yes & Yes & 386.5 & $2.62 \mathrm{E}+07$ & 397.2 & $*$ \\
\hline ems.C.100 & Yes & No & No & Yes & No & No & Yes & Yes & No & No & Yes & 555.0 & $2.54 \mathrm{E}+07$ & 379.0 & $*$ \\
\hline ems.C.101 & Yes & Yes & No & Yes & No & No & Yes & Yes & No & No & Yes & 568.4 & $2.20 \mathrm{E}+07$ & 401.8 & $*$ \\
\hline ems.C.102 & Yes & No & Yes & Yes & No & No & Yes & Yes & No & Yes & Yes & 515.2 & $9.92 \mathrm{E}+06$ & 388.3 & $*$ \\
\hline ems.C.103 & Yes & Yes & Yes & Yes & No & No & Yes & Yes & No & No & Yes & 521.3 & $9.72 \mathrm{E}+06$ & 393.2 & $*$ \\
\hline ems.C.104 & Yes & No & No & No & Yes & No & Yes & Yes & No & No & Yes & 335.8 & $1.12 \mathrm{E}+07$ & 601.7 & $*$ \\
\hline ems.C.105 & Yes & Yes & No & No & Yes & No & Yes & Yes & No & No & Yes & 335.3 & $1.24 \mathrm{E}+07$ & 618.3 & $*$ \\
\hline ems.C.106 & Yes & No & Yes & No & Yes & No & Yes & Yes & No & Yes & Yes & 341.2 & $9.34 \mathrm{E}+06$ & 392.3 & $*$ \\
\hline ems.C.107 & Yes & Yes & Yes & No & Yes & No & Yes & Yes & No & Yes & Yes & 334.8 & $9.11 \mathrm{E}+06$ & 378.6 & $*$ \\
\hline
\end{tabular}




\begin{tabular}{|c|c|c|c|c|c|c|c|c|c|c|c|c|c|c|c|}
\hline Model & $\underset{\mathbb{d}}{\stackrel{2}{2}}$ & 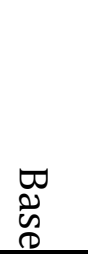 & 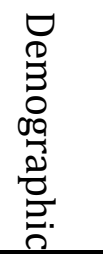 & 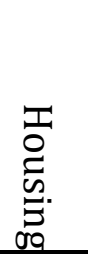 & $\begin{array}{l}\vec{D} \\
\mathbb{D} \\
\vec{D} \\
0 \\
0 \\
0 \\
D\end{array}$ & D & 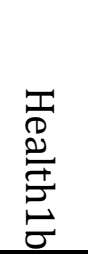 & 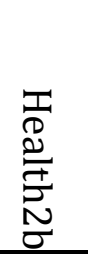 & 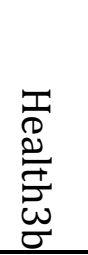 & 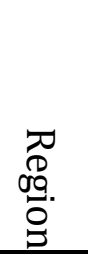 & 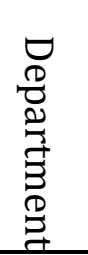 & $\mathrm{S}$ & $\mathrm{L}$ & $\mathrm{C}$ & Notes \\
\hline ems.C.108 & Yes & No & No & Yes & Yes & No & Yes & Yes & No & No & Yes & 373.0 & $1.97 \mathrm{E}+07$ & 450.5 & $*$ \\
\hline ems.C.109 & Yes & Yes & No & Yes & Yes & No & Yes & Yes & No & No & Yes & 368.5 & $1.87 \mathrm{E}+07$ & 648.6 & $*$ \\
\hline ems.C.110 & Yes & No & Yes & Yes & Yes & No & Yes & Yes & No & Yes & Yes & 409.2 & $8.51 \mathrm{E}+06$ & 377.7 & $*$ \\
\hline ems.C.111 & Yes & Yes & Yes & Yes & Yes & No & Yes & Yes & No & Yes & Yes & 394.6 & $7.68 \mathrm{E}+06$ & 376.9 & $*$ \\
\hline ems.C.112 & Yes & No & No & No & No & Yes & Yes & Yes & No & No & Yes & 353.5 & $2.40 \mathrm{E}+07$ & 434.1 & $*$ \\
\hline ems.C.113 & Yes & Yes & No & No & No & Yes & Yes & Yes & No & No & Yes & 351.8 & $1.91 \mathrm{E}+07$ & 464.1 & $*$ \\
\hline ems.C.114 & Yes & No & Yes & No & No & Yes & Yes & Yes & No & No & Yes & 916.6 & $1.50 \mathrm{E}+07$ & 577.4 & $*$ \\
\hline ems.C.115 & Yes & Yes & Yes & No & No & Yes & Yes & Yes & No & Yes & Yes & 531.9 & $1.62 \mathrm{E}+07$ & 452.4 & $*$ \\
\hline ems.C.116 & Yes & No & No & Yes & No & Yes & Yes & Yes & No & No & Yes & 533.2 & $2.85 E+07$ & 398.6 & $*$ \\
\hline ems.C.117 & Yes & Yes & No & Yes & No & Yes & Yes & Yes & No & No & Yes & 542.5 & $2.40 \mathrm{E}+07$ & 428.2 & $*$ \\
\hline ems.C.118 & Yes & No & Yes & Yes & No & Yes & Yes & Yes & No & Yes & Yes & 477.2 & $8.53 E+06$ & 406.2 & $*$ \\
\hline ems.C.119 & Yes & Yes & Yes & Yes & No & Yes & Yes & Yes & No & Yes & Yes & 476.9 & $8.27 \mathrm{E}+06$ & 410.5 & $*$ \\
\hline ems.C.120 & Yes & No & No & No & Yes & Yes & Yes & Yes & No & No & Yes & 337.3 & $1.15 \mathrm{E}+07$ & 753.2 & $*$ \\
\hline ems.C.121 & Yes & Yes & No & No & Yes & Yes & Yes & Yes & No & No & Yes & 336.8 & $1.20 \mathrm{E}+07$ & 837.3 & $*$ \\
\hline ems.C.122 & Yes & No & Yes & No & Yes & Yes & Yes & Yes & No & Yes & Yes & 365.4 & $7.67 \mathrm{E}+06$ & 444.9 & $*$ \\
\hline ems.C.123 & Yes & Yes & Yes & No & Yes & Yes & Yes & Yes & No & Yes & Yes & 336.1 & $7.18 \mathrm{E}+06$ & 411.7 & $*$ \\
\hline ems.C.124 & Yes & No & No & Yes & Yes & Yes & Yes & Yes & No & No & Yes & 378.6 & $2.33 \mathrm{E}+07$ & 488.4 & $*$ \\
\hline ems.C.125 & Yes & Yes & No & Yes & Yes & Yes & Yes & Yes & No & No & Yes & 378.5 & $2.07 \mathrm{E}+07$ & 764.5 & $*$ \\
\hline ems.C.126 & Yes & No & Yes & Yes & Yes & Yes & Yes & Yes & No & Yes & Yes & 406.8 & $7.72 \mathrm{E}+06$ & 408.0 & $*$ \\
\hline ems.C.127 & Yes & Yes & Yes & Yes & Yes & Yes & Yes & Yes & No & Yes & Yes & 395.1 & $6.95 E+06$ & 407.7 & $*$ \\
\hline ems.C.128 & Yes & No & No & No & No & No & No & No & Yes & No & Yes & 356.3 & $7.05 \mathrm{E}+06$ & 322.5 & $*$ \\
\hline ems.C.129 & Yes & Yes & No & No & No & No & No & No & Yes & No & Yes & 351.2 & $6.20 \mathrm{E}+06$ & 308.3 & $*$ \\
\hline ems.C.130 & Yes & No & Yes & No & No & No & No & No & Yes & No & Yes & 448.9 & $7.58 E+06$ & 296.1 & $*$ \\
\hline
\end{tabular}




\begin{tabular}{|c|c|c|c|c|c|c|c|c|c|c|c|c|c|c|c|}
\hline Model & 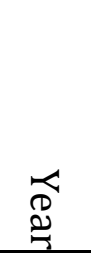 & 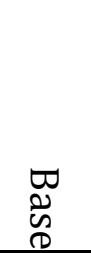 & 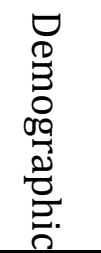 & 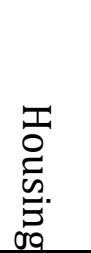 & $\begin{array}{l}\vec{D} \\
0 \\
0 \\
0 \\
0 \\
0\end{array}$ & $\begin{array}{l}T \\
\text { DD }\end{array}$ & 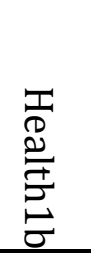 & 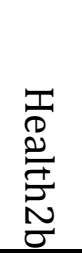 & 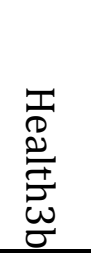 & 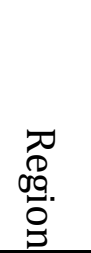 & 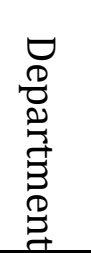 & $\mathrm{S}$ & $\mathrm{L}$ & $\mathrm{C}$ & Notes \\
\hline ems.C.131 & Yes & Yes & Yes & No & No & No & No & No & Yes & Yes & Yes & 387.4 & $3.11 \mathrm{E}+06$ & 288.4 & $*$ \\
\hline ems.C.132 & Yes & No & No & Yes & No & No & No & No & Yes & No & Yes & 550.4 & $5.45 \mathrm{E}+06$ & 278.7 & * \\
\hline ems.C.133 & Yes & Yes & No & Yes & No & No & No & No & Yes & No & Yes & 561.7 & $6.86 \mathrm{E}+06$ & 280.5 & * \\
\hline ems.C.134 & Yes & No & Yes & Yes & No & No & No & No & Yes & Yes & Yes & 518.1 & $2.42 \mathrm{E}+06$ & 296.8 & * \\
\hline ems.C.135 & Yes & Yes & Yes & Yes & No & No & No & No & Yes & Yes & Yes & 525.3 & $4.32 \mathrm{E}+06$ & 295.1 & $*$ \\
\hline ems.C.136 & Yes & No & No & No & Yes & No & No & No & Yes & No & Yes & 335.6 & $1.92 \mathrm{E}+07$ & 353.3 & * \\
\hline ems.C.137 & Yes & Yes & No & No & Yes & No & No & No & Yes & No & Yes & 335.0 & $1.86 \mathrm{E}+07$ & 353.4 & * \\
\hline ems.C.138 & Yes & No & Yes & No & Yes & No & No & No & Yes & Yes & Yes & 341.6 & $2.03 E+06$ & 307.4 & * \\
\hline ems.C.139 & Yes & Yes & Yes & No & Yes & No & No & No & Yes & Yes & Yes & 335.0 & $3.79 \mathrm{E}+06$ & 289.7 & * \\
\hline ems.C.140 & Yes & No & No & Yes & Yes & No & No & No & Yes & No & Yes & 372.3 & $4.55 \mathrm{E}+06$ & 301.3 & * \\
\hline ems.C.141 & Yes & Yes & No & Yes & Yes & No & No & No & Yes & No & Yes & 367.2 & $6.03 \mathrm{E}+06$ & 364.2 & $*$ \\
\hline ems.C.142 & Yes & No & Yes & Yes & Yes & No & No & No & Yes & Yes & Yes & 409.1 & $1.66 \mathrm{E}+06$ & 316.1 & * \\
\hline ems.C.143 & Yes & Yes & Yes & Yes & Yes & No & No & No & Yes & Yes & Yes & 394.2 & $4.81 \mathrm{E}+06$ & 310.2 & * \\
\hline ems.C.144 & Yes & No & No & No & No & Yes & No & No & Yes & No & Yes & 353.6 & $5.01 \mathrm{E}+06$ & 308.5 & * \\
\hline ems.C.145 & Yes & Yes & No & No & No & Yes & No & No & Yes & No & Yes & 351.8 & $2.27 \mathrm{E}+07$ & 306.6 & * \\
\hline ems.C.146 & Yes & No & Yes & No & No & Yes & No & No & Yes & Yes & Yes & 931.0 & $5.94 \mathrm{E}+06$ & 298.4 & * \\
\hline ems.C.147 & Yes & Yes & Yes & No & No & Yes & No & No & Yes & Yes & Yes & 534.7 & $3.39 \mathrm{E}+06$ & 290.0 & * \\
\hline ems.C.148 & Yes & No & No & Yes & No & Yes & No & No & Yes & No & Yes & 528.4 & $2.98 \mathrm{E}+06$ & 279.6 & * \\
\hline ems.C.149 & Yes & Yes & No & Yes & No & Yes & No & No & Yes & No & Yes & 535.9 & $3.96 \mathrm{E}+06$ & 281.7 & * \\
\hline ems.C.150 & Yes & No & Yes & Yes & No & Yes & No & No & Yes & Yes & Yes & 477.0 & $1.37 \mathrm{E}+06$ & 295.8 & * \\
\hline ems.C.151 & Yes & Yes & Yes & Yes & No & Yes & No & No & Yes & Yes & Yes & 478.1 & $1.40 \mathrm{E}+06$ & 293.4 & * \\
\hline ems.C.152 & Yes & No & No & No & Yes & Yes & No & No & Yes & No & Yes & 337.0 & $1.96 \mathrm{E}+07$ & 355.4 & * \\
\hline ems.C.153 & Yes & Yes & No & No & Yes & Yes & No & No & Yes & No & Yes & 336.5 & $1.94 \mathrm{E}+07$ & 374.0 & $*$ \\
\hline
\end{tabular}




\begin{tabular}{|c|c|c|c|c|c|c|c|c|c|c|c|c|c|c|c|}
\hline Model & 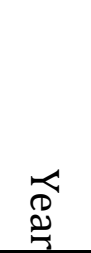 & 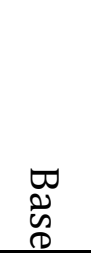 & 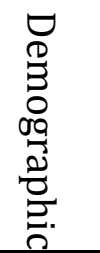 & 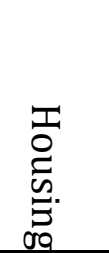 & $\begin{array}{l}\overrightarrow{0} \\
0 \\
0 \\
0 \\
0 \\
0\end{array}$ & $\begin{array}{l}T \\
\vec{D} \\
\end{array}$ & 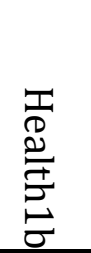 & 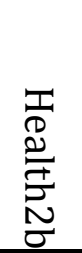 & 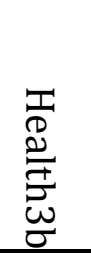 & 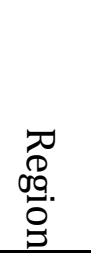 & 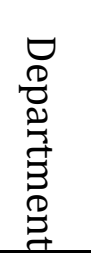 & $\mathrm{S}$ & $\mathrm{L}$ & $\mathrm{C}$ & Notes \\
\hline ems.C.154 & Yes & No & Yes & No & Yes & Yes & No & No & Yes & Yes & Yes & 366.5 & $1.60 \mathrm{E}+06$ & 317.5 & $*$ \\
\hline ems.C.155 & Yes & Yes & Yes & No & Yes & Yes & No & No & Yes & Yes & Yes & 336.3 & $2.67 \mathrm{E}+06$ & 296.7 & * \\
\hline ems.C.156 & Yes & No & No & Yes & Yes & Yes & No & No & Yes & No & Yes & 377.6 & $2.23 \mathrm{E}+06$ & 303.3 & $*$ \\
\hline ems.C.157 & Yes & Yes & No & Yes & Yes & Yes & No & No & Yes & No & Yes & 376.7 & $2.46 \mathrm{E}+06$ & 372.9 & * \\
\hline ems.C.158 & Yes & No & Yes & Yes & Yes & Yes & No & No & Yes & Yes & Yes & 405.3 & $7.80 \mathrm{E}+05$ & 325.5 & * \\
\hline ems.C.159 & Yes & Yes & Yes & Yes & Yes & Yes & No & No & Yes & Yes & Yes & 393.6 & $1.01 \mathrm{E}+06$ & 319.9 & * \\
\hline ems.C.160 & Yes & No & No & No & No & No & Yes & No & Yes & No & Yes & 356.9 & $8.29 \mathrm{E}+04$ & 32442.0 & * \\
\hline ems.C.161 & Yes & Yes & No & No & No & No & Yes & No & Yes & No & Yes & 351.6 & $1.33 \mathrm{E}+05$ & 11700.6 & * \\
\hline ems.C.162 & Yes & No & Yes & No & No & No & Yes & No & Yes & Yes & Yes & 451.5 & $9.42 \mathrm{E}+04$ & 11182.6 & * \\
\hline ems.C.163 & Yes & Yes & Yes & No & No & No & Yes & No & Yes & Yes & Yes & 388.7 & $8.98 \mathrm{E}+04$ & 9358.3 & * \\
\hline ems.C.164 & Yes & No & No & Yes & No & No & Yes & No & Yes & No & Yes & 563.5 & $3.25 \mathrm{E}+04$ & 9708.7 & * \\
\hline ems.C.165 & Yes & Yes & No & Yes & No & No & Yes & No & Yes & Yes & Yes & 574.8 & $2.85 \mathrm{E}+04$ & 11129.2 & * \\
\hline ems.C.166 & Yes & No & Yes & Yes & No & No & Yes & No & Yes & Yes & Yes & 521.2 & $2.29 \mathrm{E}+04$ & 19123.3 & * \\
\hline ems.C.167 & Yes & Yes & Yes & Yes & No & No & Yes & No & Yes & Yes & Yes & 528.8 & $2.16 \mathrm{E}+04$ & 21076.7 & * \\
\hline ems.C.168 & Yes & No & No & No & Yes & No & Yes & No & Yes & No & Yes & 336.4 & $5.85 \mathrm{E}+04$ & 6467.6 & * \\
\hline ems.C.169 & Yes & Yes & No & No & Yes & No & Yes & No & Yes & No & Yes & 335.8 & $6.41 \mathrm{E}+04$ & 6658.2 & * \\
\hline ems.C.170 & Yes & No & Yes & No & Yes & No & Yes & No & Yes & Yes & Yes & 342.6 & $1.09 \mathrm{E}+05$ & 7070.4 & * \\
\hline ems.C.171 & Yes & Yes & Yes & No & Yes & No & Yes & No & Yes & Yes & Yes & 335.8 & $8.42 \mathrm{E}+04$ & 6508.6 & $*$ \\
\hline ems.C.172 & Yes & No & No & Yes & Yes & No & Yes & No & Yes & No & Yes & 375.1 & $4.95 \mathrm{E}+04$ & 8784.1 & * \\
\hline ems.C.173 & Yes & Yes & No & Yes & Yes & No & Yes & No & Yes & No & Yes & 369.7 & $3.36 \mathrm{E}+04$ & 8370.4 & * \\
\hline ems.C.174 & Yes & No & Yes & Yes & Yes & No & Yes & No & Yes & Yes & Yes & 411.0 & $4.05 \mathrm{E}+04$ & 11576.5 & * \\
\hline ems.C.175 & Yes & Yes & Yes & Yes & Yes & No & Yes & No & Yes & Yes & Yes & 395.8 & $4.03 \mathrm{E}+04$ & 12128.6 & * \\
\hline ems.C.176 & Yes & No & No & No & No & Yes & Y & No & Yes & No & Yes & 354.1 & $9.75 E+04$ & 24638.5 & * \\
\hline
\end{tabular}




\begin{tabular}{|c|c|c|c|c|c|c|c|c|c|c|c|c|c|c|c|}
\hline Model & $\begin{array}{l}\overleftrightarrow{\mathbb{0}} \\
\stackrel{0}{2}\end{array}$ & 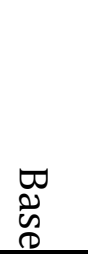 & 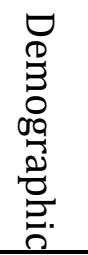 & 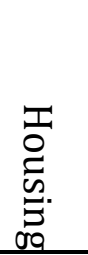 & $\begin{array}{l}\vec{D} \\
\mathbb{D} \\
\vec{D} \\
0 \\
0 \\
0 \\
D\end{array}$ & $\begin{array}{l}\text { D } \\
\mathbb{D}\end{array}$ & 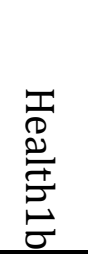 & 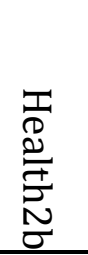 & 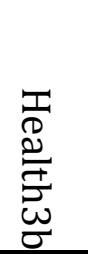 & 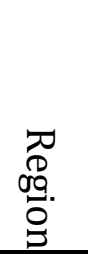 & 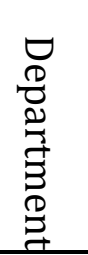 & $\mathrm{S}$ & $\mathrm{L}$ & $\mathrm{C}$ & Notes \\
\hline ems.C.177 & Yes & Yes & No & No & No & Yes & Yes & No & Yes & Yes & Yes & 352.4 & $1.43 E+05$ & 13609.0 & $*$ \\
\hline ems.C.178 & Yes & No & Yes & No & No & Yes & Yes & No & Yes & Yes & Yes & 934.0 & $1.16 \mathrm{E}+05$ & 11892.9 & $*$ \\
\hline ems.C.179 & Yes & Yes & Yes & No & No & Yes & Yes & No & Yes & Yes & Yes & 537.0 & $1.19 \mathrm{E}+05$ & 9768.3 & $*$ \\
\hline ems.C.180 & Yes & No & No & Yes & No & Yes & Yes & No & Yes & No & Yes & 540.6 & $4.07 E+04$ & 10210.0 & $*$ \\
\hline ems.C.181 & Yes & Yes & No & Yes & No & Yes & Yes & No & Yes & No & Yes & 548.1 & $3.76 \mathrm{E}+04$ & 12234.2 & $*$ \\
\hline ems.C.182 & Yes & No & Yes & Yes & No & Yes & Yes & No & Yes & Yes & Yes & 478.9 & $3.41 \mathrm{E}+04$ & 20539.6 & $*$ \\
\hline ems.C.183 & Yes & Yes & Yes & Yes & No & Yes & Yes & No & Yes & Yes & Yes & 479.9 & $3.31 \mathrm{E}+04$ & 22560.9 & $*$ \\
\hline ems.C.184 & Yes & No & No & No & Yes & Yes & Yes & No & Yes & No & Yes & 338.0 & $5.54 \mathrm{E}+04$ & 9509.2 & $*$ \\
\hline ems.C.185 & Yes & Yes & No & No & Yes & Yes & Yes & No & Yes & No & Yes & 337.5 & $6.27 \mathrm{E}+04$ & 9756.5 & $*$ \\
\hline ems.C.186 & Yes & No & Yes & No & Yes & Yes & Yes & No & Yes & Yes & Yes & 367.7 & $1.05 \mathrm{E}+05$ & 7423.0 & $*$ \\
\hline ems.C.187 & Yes & Yes & Yes & No & Yes & Yes & Yes & No & Yes & Yes & Yes & 337.2 & $8.61 \mathrm{E}+04$ & 6169.5 & $*$ \\
\hline ems.C.188 & Yes & No & No & Yes & Yes & Yes & Yes & No & Yes & Yes & Yes & 380.9 & $6.01 \mathrm{E}+04$ & 10835.9 & $*$ \\
\hline ems.C.189 & Yes & Yes & No & Yes & Yes & Yes & Yes & No & Yes & Yes & Yes & 380.0 & $4.29 E+04$ & 10190.2 & $*$ \\
\hline ems.C.190 & Yes & No & Yes & Yes & Yes & Yes & Yes & No & Yes & Yes & Yes & 407.2 & $5.13 E+04$ & 10806.8 & $*$ \\
\hline ems.C.191 & Yes & Yes & Yes & Yes & Yes & Yes & Yes & No & Yes & Yes & Yes & 395.4 & $5.12 \mathrm{E}+04$ & 11372.3 & $*$ \\
\hline ems.C.192 & Yes & No & No & No & No & No & No & Yes & Yes & No & Yes & 358.5 & $1.95 \mathrm{E}+05$ & 295.7 & $*$ \\
\hline ems.C.193 & Yes & Yes & No & No & No & No & No & Yes & Yes & No & Yes & 352.7 & $2.31 \mathrm{E}+05$ & 285.5 & $*$ \\
\hline ems.C.194 & Yes & No & Yes & No & No & No & No & Yes & Yes & Yes & Yes & 453.0 & $2.42 \mathrm{E}+05$ & 284.4 & $*$ \\
\hline ems.C.195 & Yes & Yes & Yes & No & No & No & No & Yes & Yes & Yes & Yes & 390.0 & $2.12 \mathrm{E}+05$ & 282.3 & $*$ \\
\hline ems.C.196 & Yes & No & No & Yes & No & No & No & Yes & Yes & No & Yes & 565.9 & $1.09 \mathrm{E}+05$ & 271.9 & $*$ \\
\hline ems.C.197 & Yes & Yes & No & Yes & No & No & No & Yes & Yes & No & Yes & 575.1 & $1.44 \mathrm{E}+05$ & 270.7 & $*$ \\
\hline ems.C.198 & Yes & No & Yes & Yes & No & No & No & Yes & Yes & Yes & Yes & 523.4 & $1.46 \mathrm{E}+05$ & 269.6 & $*$ \\
\hline ems.C.199 & Yes & Yes & Yes & Yes & No & No & No & Yes & Yes & Yes & Yes & 530.9 & $1.67 E+05$ & 269.5 & $*$ \\
\hline
\end{tabular}




\begin{tabular}{|c|c|c|c|c|c|c|c|c|c|c|c|c|c|c|c|}
\hline Model & $\begin{array}{l}\overleftrightarrow{\mathbb{0}} \\
\stackrel{0}{2}\end{array}$ & 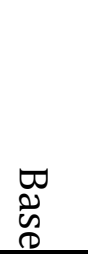 & 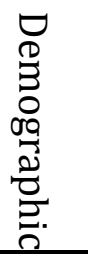 & 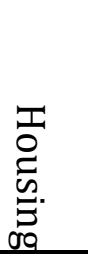 & $\begin{array}{l}\vec{D} \\
0 \\
0 \\
0 \\
0 \\
0 \\
0 \\
\end{array}$ & $\begin{array}{l}\mathbb{D} \\
\mathbb{\Phi} \\
\end{array}$ & $\begin{array}{l}\stackrel{T}{D} \\
\stackrel{D}{D} \\
\stackrel{\vec{D}}{\Xi} \\
\stackrel{\sigma}{\sigma}\end{array}$ & 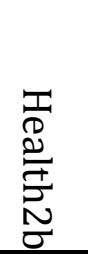 & 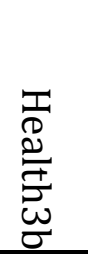 & 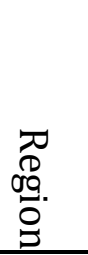 & 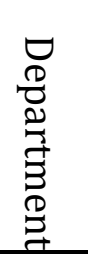 & $S$ & $\mathrm{~L}$ & $\mathrm{C}$ & Notes \\
\hline ems.C.200 & Yes & No & No & No & Yes & No & No & Yes & Yes & No & Yes & 337.4 & $7.36 \mathrm{E}+04$ & 277.1 & $*$ \\
\hline ems.C.201 & Yes & Yes & No & No & Yes & No & No & Yes & Yes & Yes & Yes & 336.8 & $6.78 \mathrm{E}+04$ & 275.2 & $*$ \\
\hline ems.C.202 & Yes & No & Yes & No & Yes & No & No & Yes & Yes & Yes & Yes & 344.3 & $9.89 \mathrm{E}+04$ & 275.2 & $*$ \\
\hline ems.C.203 & Yes & Yes & Yes & No & Yes & No & No & Yes & Yes & Yes & Yes & 336.7 & $9.08 \mathrm{E}+04$ & 270.8 & $*$ \\
\hline ems.C.204 & Yes & No & No & Yes & Yes & No & No & Yes & Yes & No & Yes & 375.7 & $9.17 \mathrm{E}+04$ & 265.0 & $*$ \\
\hline ems.C.205 & Yes & Yes & No & Yes & Yes & No & No & Yes & Yes & No & Yes & 370.7 & $9.79 \mathrm{E}+04$ & 261.4 & $*$ \\
\hline ems.C.206 & Yes & No & Yes & Yes & Yes & No & No & Yes & Yes & Yes & Yes & 413.0 & $8.26 \mathrm{E}+04$ & 262.0 & $*$ \\
\hline ems.C.207 & Yes & Yes & Yes & Yes & Yes & No & No & Yes & Yes & Yes & Yes & 397.6 & $8.41 \mathrm{E}+04$ & 262.8 & $*$ \\
\hline ems.C.208 & Yes & No & No & No & No & Yes & No & Yes & Yes & No & Yes & 355.0 & $2.15 \mathrm{E}+05$ & 292.3 & $*$ \\
\hline ems.C.209 & Yes & Yes & No & No & No & Yes & No & Yes & Yes & No & Yes & 353.9 & $3.65 E+05$ & 292.6 & $*$ \\
\hline ems.C.210 & Yes & No & Yes & No & No & Yes & No & Yes & Yes & Yes & Yes & 945.4 & $1.66 \mathrm{E}+05$ & 292.5 & $*$ \\
\hline ems.C.211 & Yes & Yes & Yes & No & No & Yes & No & Yes & Yes & Yes & Yes & 543.5 & $1.41 \mathrm{E}+05$ & 287.2 & $*$ \\
\hline ems.C.212 & Yes & No & No & Yes & No & Yes & No & Yes & Yes & No & Yes & 543.1 & $1.15 \mathrm{E}+05$ & 276.6 & $*$ \\
\hline ems.C.213 & Yes & Yes & No & Yes & No & Yes & No & Yes & Yes & No & Yes & 548.5 & $1.83 \mathrm{E}+05$ & 273.3 & $*$ \\
\hline ems.C.214 & Yes & No & Yes & Yes & No & Yes & No & Yes & Yes & Yes & Yes & 482.7 & $1.02 \mathrm{E}+05$ & 275.4 & $*$ \\
\hline ems.C.215 & Yes & Yes & Yes & Yes & No & Yes & No & Yes & Yes & Yes & Yes & 484.5 & $9.66 \mathrm{E}+04$ & 274.6 & $*$ \\
\hline ems.C.216 & Yes & No & No & No & Yes & Yes & No & Yes & Yes & No & Yes & 338.7 & $9.32 \mathrm{E}+04$ & 280.5 & $*$ \\
\hline ems.C.217 & Yes & Yes & No & No & Yes & Yes & No & Yes & Yes & No & Yes & 338.2 & $9.86 \mathrm{E}+04$ & 277.8 & $*$ \\
\hline ems.C.218 & Yes & No & Yes & No & Yes & Yes & No & Yes & Yes & Yes & Yes & 370.9 & $8.35 E+04$ & 283.3 & $*$ \\
\hline ems.C.219 & Yes & Yes & Yes & No & Yes & Yes & No & Yes & Yes & Yes & Yes & 338.5 & $7.90 \mathrm{E}+04$ & 274.9 & $*$ \\
\hline ems.C.220 & Yes & No & No & Yes & Yes & Yes & No & Yes & Yes & No & Yes & 381.5 & $8.82 \mathrm{E}+04$ & 267.6 & $*$ \\
\hline ems.C.221 & Yes & Yes & No & Yes & Yes & Yes & No & Yes & Yes & No & Yes & 380.8 & $9.98 \mathrm{E}+04$ & 263.7 & $*$ \\
\hline ems.C.222 & Yes & No & Yes & Yes & Yes & Yes & No & Yes & Yes & Yes & Yes & 409.3 & $8.89 E+04$ & 266.8 & * \\
\hline
\end{tabular}




\begin{tabular}{|c|c|c|c|c|c|c|c|c|c|c|c|c|c|c|c|}
\hline Model & 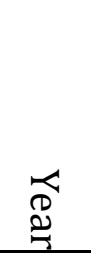 & 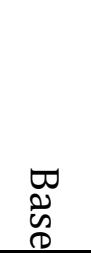 & 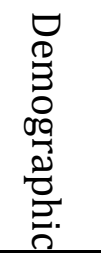 & 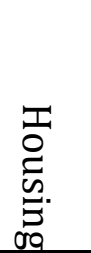 & $\begin{array}{l}\vec{D} \\
0 \\
0 \\
0 \\
0 \\
0\end{array}$ & $\begin{array}{l} \pm \\
D \\
D\end{array}$ & 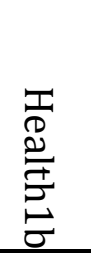 & 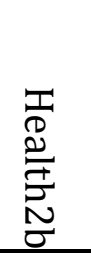 & 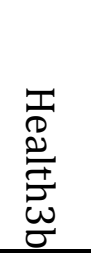 & 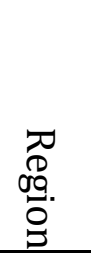 & 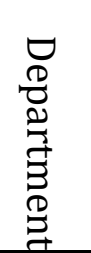 & $\mathrm{S}$ & $\mathrm{L}$ & $\mathrm{C}$ & Notes \\
\hline ems.C.223 & Yes & Yes & Yes & Yes & Yes & Yes & No & Yes & Yes & Yes & Yes & 397.3 & $5.92 \mathrm{E}+04$ & 267.1 & $*$ \\
\hline ems.C.224 & Yes & No & No & No & No & No & Yes & Yes & Yes & No & Yes & 358.9 & $3.15 \mathrm{E}+02$ & 294.4 & * \\
\hline ems.C.225 & Yes & Yes & No & No & No & No & Yes & Yes & Yes & No & Yes & 353.4 & $3.02 \mathrm{E}+02$ & 286.3 & $*$ \\
\hline ems.C.226 & Yes & No & Yes & No & No & No & Yes & Yes & Yes & Yes & Yes & 452.9 & $2.95 \mathrm{E}+02$ & 288.2 & * \\
\hline ems.C.227 & Yes & Yes & Yes & No & No & No & Yes & Yes & Yes & Yes & Yes & 390.4 & $2.87 \mathrm{E}+02$ & 283.4 & * \\
\hline ems.C.228 & Yes & No & No & Yes & No & No & Yes & Yes & Yes & No & Yes & 573.4 & $3.39 \mathrm{E}+02$ & 271.2 & * \\
\hline ems.C.229 & Yes & Yes & No & Yes & No & No & Yes & Yes & Yes & No & Yes & 582.0 & $3.22 \mathrm{E}+02$ & 270.1 & * \\
\hline ems.C.230 & Yes & No & Yes & Yes & No & No & Yes & Yes & Yes & Yes & Yes & 524.4 & $3.29 \mathrm{E}+02$ & 264.1 & * \\
\hline ems.C.231 & Yes & Yes & Yes & Yes & No & No & Yes & Yes & Yes & Yes & Yes & 531.4 & $3.07 \mathrm{E}+02$ & 261.7 & $*$ \\
\hline ems.C.232 & Yes & No & No & No & Yes & No & Yes & Yes & Yes & No & Yes & 339.8 & $5.12 \mathrm{E}+02$ & 286.2 & * \\
\hline ems.C.233 & Yes & Yes & No & No & Yes & No & Yes & Yes & Yes & No & Yes & 339.1 & $2.83 \mathrm{E}+02$ & 280.9 & * \\
\hline ems.C.234 & Yes & No & Yes & No & Yes & No & Yes & Yes & Yes & Yes & Yes & 346.7 & $3.37 \mathrm{E}+02$ & 272.7 & * \\
\hline ems.C.235 & Yes & Yes & Yes & No & Yes & No & Yes & Yes & Yes & Yes & Yes & 338.7 & $3.38 \mathrm{E}+02$ & 269.6 & * \\
\hline ems.C.236 & Yes & No & No & Yes & Yes & No & Yes & Yes & Yes & Yes & Yes & 379.1 & $2.98 \mathrm{E}+02$ & 275.3 & * \\
\hline ems.C.237 & Yes & Yes & No & Yes & Yes & No & Yes & Yes & Yes & Yes & Yes & 374.8 & $2.95 \mathrm{E}+02$ & 268.7 & * \\
\hline ems.C.238 & Yes & No & Yes & Yes & Yes & No & Yes & Yes & Yes & Yes & Yes & 415.3 & $3.48 \mathrm{E}+02$ & 260.4 & * \\
\hline ems.C.239 & Yes & Yes & Yes & Yes & Yes & No & Yes & Yes & Yes & Yes & Yes & 399.9 & $3.31 \mathrm{E}+02$ & 259.3 & * \\
\hline ems.C. 240 & Yes & No & No & No & No & Yes & Yes & Yes & Yes & No & Yes & 355.7 & $3.37 \mathrm{E}+02$ & 287.2 & $*$ \\
\hline ems.C.241 & Yes & Yes & No & No & No & Yes & Yes & Yes & Yes & No & Yes & 355.2 & $2.96 \mathrm{E}+02$ & 285.9 & * \\
\hline ems.C.242 & Yes & No & Yes & No & No & Yes & Yes & Yes & Yes & Yes & Yes & 938.3 & $3.07 \mathrm{E}+02$ & 287.6 & * \\
\hline ems.C.243 & Yes & Yes & Yes & No & No & Yes & Yes & Yes & Yes & Yes & Yes & 541.4 & $2.95 \mathrm{E}+02$ & 282.8 & * \\
\hline ems.C.244 & Yes & No & No & Yes & No & Yes & Yes & Yes & Yes & No & Yes & 550.4 & $3.37 \mathrm{E}+02$ & 272.1 & * \\
\hline ems.C.245 & Yes & Yes & No & Yes & No & Yes & Yes & Yes & Yes & No & Yes & 555.2 & $3.16 \mathrm{E}+02$ & 271.0 & * \\
\hline
\end{tabular}




\begin{tabular}{|c|c|c|c|c|c|c|c|c|c|c|c|c|c|c|c|}
\hline Model & $\begin{array}{l}\overparen{8} \\
\stackrel{0}{7} \\
\end{array}$ & 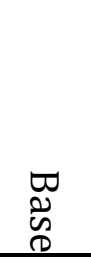 & 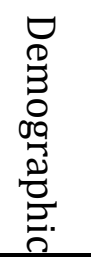 & 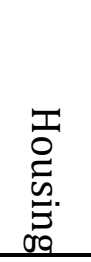 & $\begin{array}{l}\vec{D} \\
0 \\
0 \\
0 \\
0 \\
\end{array}$ & $\begin{array}{l}\text { Tే } \\
\text { D. } \\
\end{array}$ & 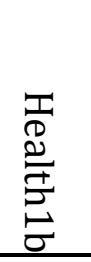 & 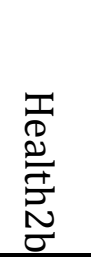 & 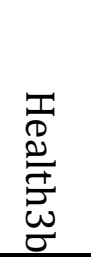 & 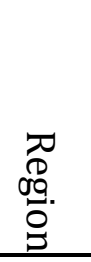 & 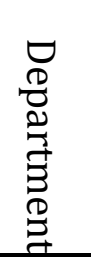 & $\mathrm{S}$ & $\mathrm{L}$ & $\mathrm{C}$ & Notes \\
\hline ems.C.246 & Yes & No & Yes & Yes & No & Yes & Yes & Yes & Yes & Yes & Yes & 484.4 & $3.30 \mathrm{E}+02$ & 265.6 & $*$ \\
\hline ems.C.247 & Yes & Yes & Yes & Yes & No & Yes & Yes & Yes & Yes & Yes & Yes & 485.8 & $3.11 \mathrm{E}+02$ & 263.2 & * \\
\hline ems.C.248 & Yes & No & No & No & Yes & Yes & Yes & Yes & Yes & No & Yes & 341.1 & $5.62 \mathrm{E}+02$ & 282.9 & $*$ \\
\hline ems.C.249 & Yes & Yes & No & No & Yes & Yes & Yes & Yes & Yes & No & Yes & 340.6 & $2.90 \mathrm{E}+02$ & 278.8 & $*$ \\
\hline ems.C.250 & Yes & No & Yes & No & Yes & Yes & Yes & Yes & Yes & Yes & Yes & 373.1 & $3.48 \mathrm{E}+02$ & 274.4 & $*$ \\
\hline ems.C.251 & Yes & Yes & Yes & No & Yes & Yes & Yes & Yes & Yes & Yes & Yes & 340.9 & $3.42 \mathrm{E}+02$ & 269.2 & $*$ \\
\hline ems.C.252 & Yes & No & No & Yes & Yes & Yes & Yes & Yes & Yes & No & Yes & 385.3 & $3.08 \mathrm{E}+02$ & 274.8 & * \\
\hline ems.C.253 & Yes & Yes & No & Yes & Yes & Yes & Yes & Yes & Yes & Yes & Yes & 385.4 & $3.02 \mathrm{E}+02$ & 269.7 & * \\
\hline ems.C.254 & Yes & No & Yes & Yes & Yes & Yes & Yes & Yes & Yes & Yes & Yes & 412.2 & $3.55 \mathrm{E}+02$ & 262.5 & * \\
\hline ems.C.255 & Yes & Yes & Yes & Yes & Yes & Yes & Yes & Yes & Yes & Yes & Yes & 400.2 & $3.38 \mathrm{E}+02$ & 261.4 & * \\
\hline
\end{tabular}

* The Long and Cluster versions fail for this model for important sub groups. 